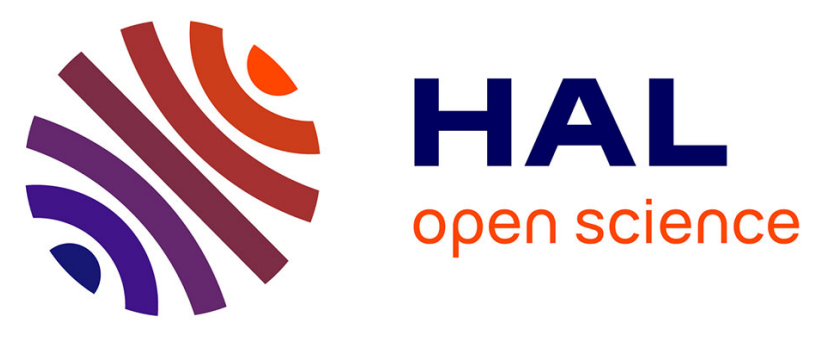

\title{
L'essor des classes moyennes dans les pays en développement et émergents : une étude comparative des enjeux d'identification, de caractérisation et de politiques publiques
}

Jean-Philippe Berrou, Matthieu Clément, François Combarnous, Dominique

Darbon, Yves-André Faure, Eric Rougier

\section{To cite this version:}

Jean-Philippe Berrou, Matthieu Clément, François Combarnous, Dominique Darbon, Yves-André Faure, et al.. L'essor des classes moyennes dans les pays en développement et émergents: une étude comparative des enjeux d'identification, de caractérisation et de politiques publiques. [Rapport de recherche] AFD - Agence Française de Développement. 2019. hal-02147531

\author{
HAL Id: hal-02147531 \\ https://hal.science/hal-02147531
}

Submitted on 12 Mar 2020

HAL is a multi-disciplinary open access archive for the deposit and dissemination of scientific research documents, whether they are published or not. The documents may come from teaching and research institutions in France or abroad, or from public or private research centers.
L'archive ouverte pluridisciplinaire HAL, est destinée au dépôt et à la diffusion de documents scientifiques de niveau recherche, publiés ou non, émanant des établissements d'enseignement et de recherche français ou étrangers, des laboratoires publics ou privés. 


\title{
Papiers de Recherche | Research Papers
}

\section{L'essor des classes moyennes dans les pays en développement et émergents : une étude comparative des enjeux d'identification, de carac térisation et de politiques publiques}

Brésil, Côte d'Ivoire, Turquie, Vietnam

\author{
J ea n-Philippe BERROU* \\ Matthieu CLÉMENT' \\ Franç ois COMBARNOUS‡ \\ Dominique DARBON\$ \\ Yves-A. FAURE** \\ Éric ROUG IER+†
}

J a nvier 2019

\begin{abstract}
Pourciter ce papier:
BERROU, J .-P., M. CLEMENT, F. COMBARNOUS, D. DARBON, Y.-A. FAURE et E. ROUGIER (2019), “L'essor des classes moyennes dans les pays en développement et émergents : une étude comparative des enjeux d'identification, de caractérisation et de politiques publiques. Brésil, Côte d'Ivoire, Turquie, Vietnam", Papiers de recherche AFD, n²019-89, J a nvier.
\end{abstract}

Contactà l'AFD :

Anda David (davida@afd.fr)

LAM (CNRS), Sciences Po Bordeaux

GREThA (CNRS), Université de Bordeaux

F REThA (CNRS), Université de Bordeaux

$\S$ LAM (CNRS), Sciences Po Bordeaux

* IRD, PRODIG (C NRS), Université Paris 1 Panthéon Sorbonne

t† GREThA (CNRS), Université de Bordeaux 


\title{
Papiers de Recherche de l'AFD
}

Les Papiers de Recherche de I'AFD ont pour but de diffuser rapidement les résultats de travaux en cours. Ils s'adressent principalement aux chercheurs, aux étudiants et au monde académique. Ils couvrent l'ensemble des sujets de travail de l'AFD : a nalyse économique, théorie économique, a nalyse des politiques publiques, sciences de l'ingénieur, sociologie, géographie et anthropologie. Une public ation dans les Papiers de Recherche de l'AFD n'en exc lut auc une autre.

L'Agence Française de Développement (AFD), institution financière publique qui met en œuvre la politique définie par le gouvemement français, agit pour combattre la pauvreté et favoriser le développement durable. Présente sur quatre continents à travers un réseau de 72 bureaux, l'AFD finance et accompagne des projets qui améliorent les conditions de vie des populations, soutiennent la croissance économique et protègent la planète. En 2014, l'AFD a consacré 8,1 milliards d'euros au financement de projets dans les pays en développement et en faveur des Outre-mer.

Les opinions exprimées dans ce papier sont celles de son (ses) auteur(s) et ne reflètent pas nécessairement celles de l'AFD. Ce document est publié sous l'entière responsabilité de son (ses) auteur(s).

Les Papiers de Recherche sont téléchargeables sur: https://www.afd.fr/fr/ressources

\section{AFD Research Papers}

AFD Research Papers are intended to rapidly disseminate findings of ongoing work and mainly target researchers, students and the wider academic community. They cover the full range of AFD work, including: economic analysis, economic theory, policy analysis, engineering sciences, sociology, geography and anthropology. AFD Research Papers and other public ations a re not mutually exclusive.

Agence Française de Développement (AFD), a public financial institution that implements the policy defined by the French Govemment, works to combat poverty and promote susta inable development. AFD operates on four continents via a network of 72 offices and finances and supports projects that improve living conditions for populations, boost economic growth and protect the planet. In 2014, AFD earmarked EUR 8.1bn to finance projects in developing countries and for overseas France.

The opinions expressed in this paper are those of the author(s) and do not necessarily reflect the position of AFD. It is therefore published under the sole responsibility of its author(s).

AFD Research Papers can be downloaded from: https://www.afd.fr/en/ressources

\author{
AFD, 5 rue Roland Barthes \\ 75598 Paris Cedex 12, France \\ ResearchPapers@afd.fr
}

ISSN 2492 - 2846 


\title{
L'essor des classes moyennes dans les pays en développement et émergents : une étude comparative des enjeux d'identification, de caractérisation et de politiques publiques. Brésil, Côte d'Ivoire, Turquie, Vietnam
}

\author{
Matthieu Clément, GREThA (CNRS), Université de Bordeaux \\ Jean-Philippe Berrou, LAM (CNRS), Sciences Po Bordeaux \\ François Combarnous, GREThA (CNRS), Université de Bordeaux \\ Dominique Darbon, LAM (CNRS), Sciences Po Bordeaux \\ Yves-A. Fauré, IRD, PRODIG (CNRS), Université Paris 1 Panthéon Sorbonne \\ Éric Rougier, GREThA (CNRS), Université de Bordeaux
}

\section{Résumé}

La densification rapide des groupes à revenu intermédiaire dans les économies en développement est certainement un événement majeur du début du $21^{\mathrm{e}}$ siècle (Kharas, 2010). Ce projet de recherche a pour objectif de proposer un examen approfondi des classes moyennes, de leurs caractéristiques, comportements, aspirations et attentes, ainsi que des implications de leur essor en matière de design des politiques publiques. L'étude porte sur quatre pays, le Brésil, la Côte d'Ivoire, la Turquie et le Vietnam, présentant des niveaux et trajectoires de développement différents. Premièrement, les résultats montrent qu'il est délicat, dans une perspective comparative, d'établir une délimitation monétaire commune des classes moyennes de revenu. Compte tenu des différences de niveau de développement économique entre les pays, les intervalles monétaires pertinents doivent être adaptés à la réalité de la distribution locale des revenus afin de rendre comparable les ménages des classes moyennes. En dépit de différences considérables de revenu, les classes moyennes s'apparentent bien à des classes de consommation, ce qui se traduit par des dépenses de consommation élevés qui se déplacent et se diversifient vers l'éducation, le logement et la santé. La relative satisfaction des besoins immédiats autorise la formulation d'aspirations à la promotion de soi et des siens, qui sont partout associées à une valorisation de l'effort individuel. Ensuite, au-delà de ces éléments de proximité, l'étude comparative souligne la forte hétérogénéité interne de chaque classe moyenne nationale, notamment au regard des différences significatives qui existent entre elles en termes de statut dans l'emploi, de niveau d'éducation ou de revenu. Chaque ensemble national se caractérise par l'existence de quatre à six groupes bien spécifiques pouvant s'expliquer par l'historicité des trajectoires propres à chaque société. Les différentes classes moyennes nationales entretiennent des rapports ambigus et complexes avec l'Etat, le pouvoir et les politiques publiques. D'un côté, la très forte hétérogénéité socio-économique des classes moyennes nationales limite leur capacité d'organisation collective et d'influence politique. D'un autre côté, l'étude constate l'absence de politique officielle et globale de promotion de la classe moyenne en tant que groupe social dont l'influence pourrait être reconnue stratégique dans la trajectoire présente et future de développement économique.

Mots-clés : Classes moyennes, politiques publiques, mobilité sociale

Classification JEL : I3, D31, O2

Version originale : Français

Acceptée : Janvier 2019 


\section{Introduction}

La densification rapide des groupes sociaux à revenu intermédiaire dans les économies en développement est certainement un événement majeur du début du 21ème siècle (Kharas, 2010). La part de la population du monde en développement constitutive de la "global middle class" (entre 2 et $13 \$$ quotidiens en PPA) serait passée de 32,7\% en 1990 à 48,5\% en 2005, et $80 \%$ de cette hausse serait imputable aux pays en développement (Ravallion, 2010). Selon les estimations de Kharas (2017), 3,2 milliards d'individus composaient cette global middle class à la fin de l'année 2016, soit 500 millions de plus que ne le laissaient supposer ses précédentes projections (Kharas, 2010). En outre, près de $80 \%$ des membres de la global middle class seraient susceptibles de vivre en dehors des pays riches d'ici 2030, principalement au sein des pays émergents et tout particulièrement asiatiques (Brunke et al., 2013).

Le terme de classe moyenne est de plus en plus utilisé pour désigner cet ensemble (très hétérogène) de ménages des pays en développement qui sont parvenus à se soustraire de la pauvreté sans pour autant être riches. L'émergence de ce groupe de population dans un grand nombre de pays en développement pourrait avoir des effets socio-économiques majeurs dépassant largement les frontières des pays concernés. La densification de la strate de revenu intermédiaire des pays en développement permettrait en effet de soutenir la croissance mondiale face au tarissement des potentialités de croissance de la consommation des classes moyennes affectant les économies développées (Kharas, 2010). En raison de leur taille et de leurs niveaux d'éducation, les classes moyennes des pays en développement pourraient également remodeler tout à la fois le marché du travail global et les scénarios de division internationale du travail des décennies futures (Freeman, 2007). Enfin, du fait de leur poids dans la population et l'économie mondiale, les comportements de consommation de ces groupes de revenu moyen sont susceptibles d'avoir des conséquences environnementales significatives, ainsi que l'illustre le cas chinois.

Pour la littérature académique, l'essor des classes moyennes des pays en développement pourrait être un facteur déterminant dans le processus d'émergence à plusieurs titres : (i) il permettrait l'expansion du marché domestique et favoriserait la croissance du PIB ; (ii) il contribuerait à la diversification de la demande sociale et de la consommation de biens et services (Banerjee et Duflo, 2008) ; (iii) il modifierait la structure sociale et révèlerait le potentiel de mobilité ascendante entre les strates de la société ; (iv) il redistribuerait enfin les enjeux politiques, en rendant possible à la fois une stabilisation politique accrue autour d'une masse importante de la population et la possibilité d'alliances alternatives favorisant l'inclusion politique (Lipset, 1959; Easterly, 2001; Loayza et al., 2012). Il est important de noter que ces effets attendus sont basés sur des hypothèses théoriques construites à partir de l'expérience historique de certains pays aujourd'hui avancés. Or, les travaux centrés sur les pays émergents contemporains n’ont pas encore délivré suffisamment de preuves empiriques, notamment au niveau microéconomique, pour valider ces intuitions. 
Ce rapport propose une analyse comparative du phénomène de densification du groupe de revenu intermédiaire dans quatre pays en développement, le Brésil, la Côte d'Ivoire, la Turquie et Vietnam, représentant les quatre grandes régions (Amérique Latine, Afrique sub-saharienne, Moyen-Orient et Asie) ${ }^{7}$. Même si ces quatre pays présentent des niveaux de développement très différents et ont suivi des trajectoires socio-économiques et politiques très contrastées, tous sont concernés, à des degrés divers, par l'expansion des groupes de revenu intermédiaire, que nous appellerons "classes moyennes", et par les effets économiques et politiques qui peuvent en résulter. La présente analyse comparative porte sur la constitution de ces groupes, sur leur transformation éventuelle en groupes sociaux d'identité et de mobilisation et sur les implications de ces évolutions en termes de politiques publiques. L'analyse comparative permettra de discuter de la pertinence de l'assimilation de ces catégories intermédiaires à des classes moyennes, c'est-àdire de vérifier l'existence d'une identité sociale. Il s'agira donc de déterminer si ces groupes partagent des comportements et croyances identiques (ou au moins convergentes) et des projets politiques et des formes de mobilisation communs. Elle permettra aussi de discuter de la pertinence et du réalisme de corrélations mises en évidence dans la littérature associant les classes moyennes à la démocratie, à l'éducation, aux valeurs de l'économie de marché ou à la redistribution.

Le projet affirme la possibilité de travailler parallèlement sur quatre pays que peu de choses rapprochent (zones géographiques, niveaux de développement, structures sociales, trajectoires historiques, systèmes de gouvernement) sans tenter a priori de poser une hypothèse de proximité, ni d'irréductibilité'. Chaque cas a fait l'objet d'études systématiques, menées avec l'appui d'équipes locales, dont l'objectif était de proposer une analyse spécifique de chaque trajectoire nationale de formation des classes moyennes et d'identifier des enjeux et des éléments communs aux quatre pays. Afin d'analyser ces enjeux de façon à la fois comparative et la plus compréhensive possible, une même approche mixte quantitative-qualitative a été mise en place pour les quatre pays. Cette approche a consisté à mobiliser successivement des données issues d'enquêtes-ménages nationales récentes, et des données originales d'enquêtes qualitatives menées dans le cadre du projet. La première enquête qualitative a été menée auprès d'un échantillon de ménages de la classe moyenne et a permis de mettre en évidence les spécificités comportementales et aspirationnelles des membres du groupe de revenu intermédiaire. La

7 Ici, la représentation ne signifie pas la représentativité. Chaque région recèle une grande diversité de situations nationales qui commencent à être documentées statistiquement. Voir notamment ESCWA (2014) pour la région Moyen-Orient et Afrique du Nord, ADB (2010) pour l'Asie, AfDB (2011) pour l'Afrique et Castellani et al. (2014) pour l'Amérique latine.

8 Le terme classe moyenne est repris ici par mesure de facilité comme synonyme de groupe à revenu intermédiaire sans lui donner de signification sociale prédéfinie, mais en retenant qu'il y a derrière cette étiquette plus qu'un phénomène statistique. Nous discuterons du contenu de ce terme plus loin dans le texte.

9 Le choix de l'échantillon de pays conduit à valoriser plutôt une approche en termes de contrastes dramatiques inspirée de la sociologie historique (Skocpol and Somers, 1980 ; Skocpol, 1984). En outre, la démarche de cette étude est associée à l'approche des comparaisons verticales, c'est-à-dire entre des cas marqués par des différences majeures d'état de la technologie et de niveau de développement au sens large, chaque situation pouvant ainsi s'inscrire dans un continuum économique commun (Thiriot, 2004). 
seconde enquête qualitative, réalisée auprès d'institutions publiques et privées, a permis d'identifier plus clairement la perception du phénomène du côté des acteurs des politiques publiques et des marchés.

Sur la base de l'exploitation de ces différentes sources d'information, cette synthèse comparative se propose de répondre à deux objectifs principaux. En premier lieu, il s'agit de clarifier le contenu et la portée de la notion de classe moyenne dans le contexte des pays en développement: (i) en étudiant la cohésion et/ou l'hétérogénéité du groupe de revenu intermédiaire dans chaque pays, (ii) en recherchant les facteurs spécifiques ou communs (histoire, politiques publiques, trajectoires socio-économiques) qui ont pu supporter l'émergence de ce(s) groupe(s) dans chaque cas, et (iii) en analysant l'existence, au-delà des circonstances historiques de leur fabrication et de leur hétérogénéité, de caractéristiques structurelles en matière de comportements, préférences et aspirations qui pourraient les différencier des autres catégories sociales. En second lieu, l'approche comparative doit permettre d'informer les acteurs des politiques publiques ciblant les groupes de revenu intermédiaire des pays en développement au moyen d'évidences empiriques mixtes à la fois plus diversifiées et systémiques que les simples intuitions et corrélations observées jusque-là par la littérature sur la question.

De manière plus générale, la présente synthèse comparative produit une discussion originale de l'utilité théorique, descriptive et politique du concept de classe moyenne dans le contexte des pays en développement qui peut se résumer en trois points.

Premièrement, la présente analyse montre que l'expression global middle class recouvre des réalités différenciées d'espaces sociaux nationaux fortement fragmentés. La composition du groupe de revenu intermédiaire présente en effet un degré d'hétérogénéité important dans les quatre pays analysés, ce qui suggérerait une certaine inconsistance de la notion de classe moyenne, utilisée au sens de classe sociale homogène, dans le contexte des pays en développement. Pourtant, le travail comparatif a permis d'identifier certains groupes socio-économiques communs aux quatre pays, tels que les retraités ou les salariés formels du secteur public et du secteur privé, qui pourraient figurer comme des éléments universels des classes moyennes, quel que soit le niveau de développement ou la trajectoire historique de développement économique et de transformation politique.

Deuxièmement, les analyses nationales et l'analyse comparative ont fait la preuve de la grande vulnérabilité de certaines composantes de la classe moyenne des quatre pays en développement de notre échantillon. L'identification de cette vulnérabilité n'est pas un résultat nouveau puisqu'il a déjà été souligné par les travaux d'économistes du développement (souvent spécialistes de la pauvreté) comme Banerjee et Duflo (2008), Ravallion (2010) ou Birdsall et al. (2014). Si la taille de cette strate vulnérable du groupe de revenu intermédiaire varie selon le niveau de développement, sa composition est assez similaire dans les quatre pays, les travailleurs et entrepreneurs individuels de l'informel constituant une partie significative de ce groupe assimilable à la "floating class" de la littérature. 
Troisièmement, cette étude montre que contrairement à ce qui est généralement attendu, les politiques publiques ciblant ou accompagnant explicitement l'essor des classes moyennes ne sont pas généralisées. En effet, malgré leurs trajectoires politiques et économiques très contrastées, les pays que nous comparons ont en commun, à l'exception récente de la Turquie, de ne pas avoir mis en place de politique publique spécifiquement ciblée sur le groupe de revenu intermédiaire et de laisser au marché le soin de répondre à leurs attentes. En revanche, la classe moyenne, ou certaines parties spécifiques de ce groupe, font l'objet d'instrumentalisations politiques dont les fins et les formes sont différentes dans les quatre pays. Enfin, cette étude a pu souligner la faible capacité de la classe moyenne à infléchir les politiques publiques, et ce quel que soit la trajectoire politique et le niveau de développement, ce qui impose une grande prudence dans la transposition de l'expression "classe moyenne" aux pays en développement.

La suite du document est structurée de la façon suivante. Dans la section 2, nous justifions l'intérêt d'analyser les groupes de revenu intermédiaire de façon comparative et précisons un certain nombre de précautions et d'options méthodologiques structurant l'analyse. Dans la section 3, nous présentons la structure des profils socio-économiques des groupes intermédiaires de revenu des quatre pays avant de comparer leurs comportements dans la section 4. Enfin, la section 5 met en contraste les besoins et aspirations et les réponses de politiques publiques et de marché et trace un certain nombre d'enjeux communs et spécifiques liés aux relations entre groupes de revenu intermédiaire et Etat dans les différents pays.

\section{Comparer les classes moyennes des pays en développement : motivations et méthodes}

\subsection{Pourquoi comparer les classes moyennes des pays en développement?}

La classe moyenne est une notion transdisciplinaire dont le contenu et la portée analytique sont controversés (Sick, 1993), et dont la transposition au contexte des pays en développement ou émergents contemporains pose un certain nombre de problèmes

Le terme "classe moyenne" est un terme valise appliqué aux pays aujourd'hui développés pour désigner la population ni riche ni pauvre, essentiellement composée de cadres intermédiaires, techniciens et fonctionnaires, qui se situait entre les classes populaires, composées d'ouvriers, employés et agriculteurs et les classes aisées comprenant le patronat et les cadres supérieurs. L'approche sociologique de la notion de classes moyennes dans le contexte des pays industrialisés a oscillé entre une interprétation considérant le potentiel politique de ce groupe social, l'interprétant notamment comme un marqueur de démocratisation, de développement économique et de libre entreprise, et une interprétation plus critique déniant à ce groupe une homogénéité socio-économique et une autonomie politique par rapport à la bourgeoisie (Bourdieu, 1979). Tiraillée entre unité et hétérogénéité, autonomie et dépendance, ou bien convergence et diversité des trajectoires historiques de stratification sociale, la sociologie n'a jamais été en mesure de définir positivement le contenu de cette notion qui reste très controversée (Sick, 1993; Bosc, 2008). 
Malgré son caractère à la fois polémique et inconsistant (voir Encadré 1), le terme a été réactivé par plusieurs catégories d'acteurs du développement global guidés par des motivations différentes. D'un côté, les cabinets de consultance ou les grandes banques privées lient clairement les classes moyennes globales à la perspective de nouveaux marchés de consommation et d'épargne. Le cabinet McKinsey a par exemple consacré un grand nombre de rapports et de notes de synthèse aux classes moyennes des économies émergentes et à leur comportement de consommation depuis une dizaine d'années (McKinsey Global Institute, 2006, 2012, 2017; Court et Narasimhan, 2010; Hoefel et al., 2015). Le McKinsey Global Institute (2017), identifie explicitement ces catégories sociales à une "consumer class". Le Crédit suisse (Crédit Suisse Research Institute, 2017) ou la banque Goldman Sachs (Wilson et Dragusanu, 2008) ont publié plusieurs rapports sur les classes moyennes émergentes, notamment en Asie, avec un double focus sur leurs dépenses et leur capacité d'épargne. En Afrique, la CFAO se focalise également sur les enjeux de consommation et de différenciation des marchés (CFAO, 2015). La Banque Merrill Lynch (Merrill Lynch Global Research, 2016) s'est intéressée aux bottom billions, c'est à dire aux individus disposant de revenus modestes (i.e. allant de 1 à 10 en PPA), mais dont le poids en matière de consommation est potentiellement considérable (5000 milliards de dollars de pouvoir d'achat et 7400 milliards de richesse selon les estimations de la banque).

D’un autre côté, les banques régionales de développement sont également très impliquées dans la fabrication de la catégorie "classe moyenne globale". Pour elles, l'enjeu est de synthétiser un ensemble de changements distributifs et productifs qui touchent les pays à revenu intermédiaire et pourraient conduire à des trappes de croissance et d'inefficience sociale s'ils n'étaient pas correctement pris en compte et accompagnés par les politiques publiques des pays concernés. Particulièrement actives sur ce front, l'Asian Development Bank (ADB, 2010 ; Chun, 2010 ; Andrianjaka, 2017), l'African Development Bank (AFDB, 2011 ; Ncumbe et Lufumpa, 2015) ou l'Inter-American Development Bank (Castellani et al., 2014 ; Stampini et al., 2015) ont ainsi pu aborder la question de l'expansion des classes moyennes régionales en adoptant le prisme des effets de cette expansion sur la dynamique de croissance économique, de transformation structurelle et d'inclusion sociale des économies en développement, ainsi que sur la transformation de la consommation, de la gouvernance et des politiques publiques.

La notion de classe moyenne a été abusivement mobilisée pour globaliser deux constats différents qui ne nécessitaient pas a priori son secours : celui de l'expansion des revenus et de la consommation de parties substantielles de la population en sortie tendancielle de pauvreté et celui de l'enrichissement stabilisé, effectif et durable des parties les plus aisées des catégories intermédiaires de revenu (Kroeber, 2016 ; Melber, 2016). Ces travaux ont ainsi généré une “controverse manufacturée", c'est-à-dire un débat très large fondé sur des catégories intuitives non validées, la capacité performative et évocatrice de la notion « classe moyenne » faisant le reste en répondant alors aux intérêts de tous, politiques et agents économiques, en quête d'émergence politique et de nouveaux marchés. 


\section{Encadré 1. Origines du terme "classes moyennes".}

L'étymologie du terme classes moyennes est révélatrice des ambiguités épistémologiques de celui-ci. Alors que le qualificatif «moyennes » renvoie à la position intermédiaire qu'occupent les individus de ce groupe dans l'échelle des revenus ou des statuts professionnels, le terme «classes » est un héritage de la sociologie marxiste et désigne la place occupée par le groupe dans les rapports sociaux de production et de pouvoir, déterminée par la propriété de moyens de production (capital ou travail). A cette dimension objective de la classe, il convient d'ajouter la dimension subjective d'affiliation ou d'identification au groupe entretenue par les interactions sociales ainsi que par les comportements et préférences partagés. La classe moyenne peut être assimilée à la " petite bourgeoisie » décrite par Marx (1887) dans le cadre du capitalisme industriel européen comme un petit groupe indépendant d'entrepreneurs et de bureaucrates qui possédaient les compétences, les connaissances et l'éducation nécessaires pour acquérir le pouvoir économique. Veblen (1899), Weber (1920 [1947]) et Halbwachs (1939) inaugureront l'analyse moderne des classes sociales, le premier en différenciant les classes inférieures et supérieures par leur mode de consommation, la logique de la classe moyenne consistant à se déplacer des consommations caractéristiques des premières vers celles caractéristiques des secondes, et les seconds en proposant un cadre d'analyse de la stratification sociale articulant les notions de classe et de statut socio-économique, de prestige et de pouvoir. Enfin, l'intégration des travailleurs salariés employés se fait au cours du 20ème siècle en parallèle de l'expansion de l'Etat providence et conduit à la notion de classe moyenne analysée par les sociologues à la suite de Weber (1920 [1947]). On peut notamment faire référence aux travaux de Mills (1951) analysant la structure par classes de la société américaine d'après-guerre par le statut dans l'emploi, le niveau d'éducation et la catégorie socioprofessionnelle, ainsi qu'à ceux de Goldthorpe popularisant la notion de service class pour la Grande Bretagne (Goldthorpe, 1980).

Les historiens et économistes du développement ont maintenu, ou réactivé, une conception de la "classe moyenne" rationnelle, modérée, entrepreneuse et économe, inspirée de la "petite bourgeoisie" du 19ème siècle qui est supposée avoir joué un rôle central dans l'émergence économique de l'Europe de l'ouest (Adelman and Morris, 1967 ; Landes, 1998 ; Doepke and Zilibotti, 2008). D'abord associée à la bourgeoisie d'affaires émergente qui aspire à accéder aux affaires publiques et à changer l'ordre économique et social, le concept de "petite bourgeoisie" s'est progressivement ouvert aux petits entrepreneurs et aux professions intellectuelles.

A une conception historiciste des classes sociales, analysées comme le produit de rapports économiques, sociaux et politiques dans le temps long, s'oppose une approche plus réductrice et essentialiste, essentiellement mobilisée par les économistes, définissant le groupe social des classes moyennes par un intervalle de revenu et ne cherchant pas à identifier la nature des relations entre les différentes classes et les différentes composantes de chaque classe. Alors que la première approche se définit intégralement dans le contexte historique des pays aujourd'hui développés, la seconde approche suppose a priori l'universalisme du concept de classe moyenne, mais propose, à travers des analyses empiriques utilisant des données micro-économiques 
d'enquêtes, une relecture critique du concept et de sa validité pour les pays en développement aujourd'hui. Le présent document s'appuie sur cette dernière approche, tout en intégrant des éléments plus dynamiques de comparaison des trajectoires historiques de formation des groupes de revenu intermédiaire dans quatre pays en développement.

Le monde académique n'est pas resté insensible à la notion de classe moyenne, avec cependant un enthousiasme bien plus mesuré. La complexité et la forte connotation performative de cette notion, la relativité de ses formes aux trajectoires nationales de stratification sociale, les incertitudes sur l'identité collective et la cohésion sociale et politique de ces groupes de revenus intermédiaires, les controverses sur les corrélations entre ces groupes et la nature des régimes politiques et des régimes de croissance, ont généré des suspicions légitimes sur l'utilisation du terme et du concept.

Les sociologues, anthropologues et politistes sont ainsi restés prudents avec la notion de classe moyenne et les mutations qui la concernent dans les pays en développement. Quelques travaux existent en Amérique latine et en Asie, mais ils restent épars (Jaffrelot, 2008; De La Calle et Rubio, 2012; Villegas, 2012). Parmi eux, certains insistent sur le caractère intergénérationnel de l'émergence des classes moyennes et de leur transformation de flux en stocks (Ferreira et al., 2013). En Afrique, mis à part l'Afrique du sud où une littérature ancienne et suivie existe, prolongée par l'enjeu de l'émergence des «Black Diamonds », les travaux sont peu nombreux, plutôt anciens et très spécifiques (Côte d'ivoire, Zambie, Kenya). Néanmoins, depuis 2010, de nouveaux travaux émergent (Darbon et Toulabor, 2014 ; Kroeber, 2016 ; Melber 2016).

De leur côté, les économistes spécialisés sur les pays en développement se sont progressivement emparés de la problématique des classes moyennes, de nombreux travaux comparatifs ayant analysé l'expansion statistique des catégories intermédiaires de revenu au cours des 10 15 dernières années (Milanovic et Yitzhaki, 2002 ; Birdsall, 2010 ; Kharas, 2010, 2017 ; Ravallion, 2010 ; Ferreira et al., 2013). La littérature économique a également produit quelques analyses statistiques et économétriques visant à identifier les transformations socio-économiques et politiques portées par l'expansion de ces catégories de revenu intermédiaire dans les pays en développement (Easterly, 2001 ; Banerjee et Duflo, 2008 ; Loayza et al., 2012). Cependant, la définition et l'identification des classes moyennes proposées dans ces études restent la plupart du temps très limitées car ne reposant que sur des critères monétaires.

Pour résumer ce bref tour d'horizon, les sciences sociales adoptent des positions critiques vis-àvis de l'usage de cette notion de classe moyenne dans le contexte des pays en développement, tout en portant un intérêt croissant aux nouvelles dynamiques sociales générées par les processus récents de sortie de pauvreté d'un grand nombre de ménages dans de nombreux pays en développement, ainsi qu'à leurs effets sur la gouvernance socio-économique et les réformes politico-institutionnelles. L'un des défis de la présente recherche a donc été d'éviter de mélanger les différentes perspectives afin d'éviter les confusions et des analyses de sens commun. Le terme 
«classes moyennes » sera retenu ici pour désigner par commodité le groupe de populations à revenu intermédiaire et mener un travail exigeant d'identification.

\subsection{Comment comparer les classes moyennes des pays en développement? Une méthode comparative mixte quantitative-qualitative}

De manière générale, il existe quatre approches de définition et d'identification des classes moyennes dans la littérature en sciences sociales. La première approche est économique et est basée sur un critère de revenu, de consommation voire de richesse, qui distingue les ménages ou individus de la classe moyenne comme étant ni pauvres ni riches. Une seconde approche, plus sociologique, est basée sur l'observation du statut social et mobilise des informations sur l'éducation, la catégorie socioprofessionnelle (CSP), le type d'emploi et la culture. Une troisième approche, plus politique, est basée sur l'observation d'éléments plus subjectifs comme la conscience de classe ou l'existence d'une identité globale basée sur des préférences et aspirations partagées pouvant conduire à des formes de mobilisation collective. Enfin, une dernière approche, managériale, repose sur la caractérisation des habitudes de consommation et de leurs trends. Il résulte de cette grande diversité des approches un usage souvent peu précautionneux et parfois confus du terme « classe moyenne».

L'analyse proposée ici est globale car elle tente d'articuler ces différentes approches en utilisant une grande variété de matériaux, à la fois quantitatif et qualitatif, mais également parce qu'elle cherche à identifier, de façon comparative, la multitude d'enjeux liés à la montée en puissance des classes moyennes dans les pays en développement. L'approche méthodologique qui a été déployée dans le cadre de cette étude comparative se décompose en trois étapes.

La première étape porte sur l'identification et la caractérisation de la classe intermédiaire de revenu et de ses différentes composantes dans les quatre pays étudiés. Cette partie du travail repose exclusivement sur l'analyse quantitative de données issues d'enquêtes ménages représentatives à l'échelle nationale. Les quatre enquêtes mobilisées dans le projet sont les suivantes : (i) l'enquête PNAD (Pesquisa Nacional por Amostra de Domicilios) de 2014 pour le Brésil ; (ii) l'enquête ENV (Enquête sur le niveau de vie des ménages) de 2015 pour la Côte d'Ivoire ; (iii) l'enquête SILC (Survey on Income and Life Conditions) de 2014 pour la Turquie ; (iv) l'enquête VHLSS (Viet Nam Household Living Standard Survey) de 2012 pour le Vietnam.

Cette phase est réalisée selon la méthodologie séquentielle développée par Bonnefond et al. (2015). Elle consiste dans un premier temps à établir pour chaque pays un intervalle de revenu pertinent permettant d'isoler une classe intermédiaire de revenus constituée de ménages non pauvres selon les critères locaux et n'appartenant pas au 5\% les plus riches de la distribution. Une fois le périmètre de la classe intermédiaire de revenu établi, son homogénéité est discutée. L’idée centrale est d'enrichir le repérage de la classe moyenne dans une perspective multidimensionnelle. Plusieurs dimensions considérées comme importantes dans la littérature pour définir la classe moyenne sont alors prises en compte, notamment le niveau d'éducation, l'occupation (CSP et 
secteur d'activité), le statut dans l'emploi et le type d'employeur et enfin l'éventuelle pluriactivité des acteurs ${ }^{10}$.

\section{Encadré 2. Méthodologie de la classification mixte.}

Après analyse des questionnaires d'enquêtes, nous avons sélectionné et recodé cinq variables liées à l'éducation et à l'emploi pour mettre en œuvre la classification : (i) le niveau d'éducation le plus élevé atteint par le chef de ménage, (ii) la catégorie socioprofessionnelle du chef de ménage, (iii) le statut dans l'emploi du chef de ménage, (iv) le type d'employeur ou secteur institutionnel du chef de ménage et (v) la pluriactivité éventuelle du chef de ménage. Sur la base de ces cinq variables, la procédure retenue effectue une classification d'un grand ensemble d'individus caractérisés par leurs premières coordonnées factorielles créées par une procédure initiale d'analyse factorielle (ici une analyse des correspondances multiples menée sur les cinq variables). Une première classification est obtenue par croisement de plusieurs partitions de base construites autour de centres mobiles, puis les classes stables ainsi formées sont agrégées par une méthode de classification hiérarchique. Le critère d'agrégation ici retenu est le critère de Ward. La partition que nous retiendrons (le nombre de groupes retenus au sein de la classe moyenne de revenu) résulte de l'analyse de l'histogramme des indices de niveau d'agrégation des nœuds de la hiérarchie et de l'analyse du dendrogramme (schéma synthétique de l'arbre d'agrégation). Sur cette base, nous proposons pour chaque pays une classification en groupes homogènes et clairement distincts les uns des autres.

Afin d'explorer l'hétérogénéité des classes moyennes de revenu d'un point de vue multidimensionnel, nous avons mis en œuvre une procédure de classification mixte (Encadré 2) sur plusieurs variables décrivant les statuts occupationnels et éducatifs des ménages de l'échantillon considéré pour chaque pays. Les différentes classes moyennes identifiées dans chaque pays sur la base d'un tel critère multidimensionnel pourront alors être caractérisées en détail au regard de différentes caractéristiques: composition démographique, âge, localisation géographique, secteurs d'activité, logement et équipement en biens durables, comportements de santé et d'éducation, transports, accès aux médias, perceptions subjectives du bien-être, etc. Outre l'objectif d'identification et de caractérisation, les résultats produits par cette première étape doivent nous conduire à questionner la pertinence du concept de classe moyenne au regard de l'hétérogénéité intra et inter-pays de cette catégorie.

La deuxième étape méthodologique vise à identifier les comportements et aspirations des différentes composantes de la classe moyenne identifiées lors de la première étape. L'originalité de cette étape est de combiner une analyse quantitative à partir des données d'enquêtes ménages

10 Il est à noter que ces dimensions convergent partiellement avec celles retenues par l'approche comparative de Thurlow et al. (2015) qui partent d'une définition a priori de la classe moyenne adaptée à l'Afrique subsaharienne sur la cooccurrence de trois critères nécessaires et suffisants: éducation secondaire, logement décent et emploi qualifié. 
de la première étape et une enquête qualitative auprès d'un échantillon de ménages de chaque segment de la classe moyenne pour chacun des pays étudiés. Dans cette perspective qualitative, une analyse systématique des comportements et aspirations des membres de la classe moyenne à partir d'entretiens qualitatifs semi-directifs a été menée dans chaque pays. Ces entretiens ont été réalisés auprès d'un échantillon restreint de 25-30 ménages issus des différents segments de la classe moyenne identifiés dans la première étape, et ce pour chacun des pays. L'objectif de cet échantillonnage par quotas est de choisir une population enquêtée couvrant l'ensemble des différents types de classes moyennes "révélés » par l'analyse quantitative. A cet effet, les entretiens qualitatifs ont été menés dans les quatre pays en deux lieux distincts : une grande métropole du pays (Rio de Janeiro, Abidjan, Istanbul et Hanoi) et une ville secondaire distante de celle-ci (Fortaleza, Soubré, Gaziantep et Nam Dinh) afin de percevoir d'éventuelles variations entre capitale et provinces.

En plus de produire une information riche sur la dynamique de la stratification sociale (mobilité intra et intergénérationnelle), ces entretiens qualitatifs ont permis d'identifier précisément en quoi l'appartenance aux différents segments des classes moyennes nationales induit des comportements et des aspirations économiques, sociales, environnementales et politiques spécifiques. Ces données ont été collectées à partir d'un certain nombre de contenus standardisés afin d'assurer la comparabilité des informations recueillies, les grilles d'entretien ayant été systématiquement adaptées aux spécificités des différents terrains observés (vocabulaire spécifique, particularités locales, etc.). Les dimensions suivantes ont fait l'objet d'une attention particulière au cours de l'enquête : (i) les aspirations économiques des classes moyennes (mobilité professionnelle, mobilité intergénérationnelle, accès au capital, accès à des services d'éducation et de santé de qualité, accès aux biens publics et collectifs, conceptions de la justice sociale (fiscalité, redistribution), accès aux opportunités, etc.) ; (ii) la participation à la vie sociale (insertion aux réseaux sociaux, implication dans la vie associative, participation aux cérémonies, participation à des activités syndicales, etc.) ; (iii) les demandes de protection sociale (existence d'une couverture maladie, d'une assurance retraite, couverture publique ou privée, etc.) ; (iv) les aspirations en termes de qualité de vie (attitude vis à vis de l'arbitrage croissance/environnement, consommation d'énergie, connexion aux infrastructures en réseaux, modes de déplacement, recherche d'aménités environnementales, etc.) ; (v) les préférences et aspirations politiques (accès aux médias, vote, participation politique, préférences politiques, confiance dans les institutions, distance à la tradition, etc.).

La troisième étape de la méthodologie porte sur la perception institutionnelle des classes moyennes et sur les implications de leur développement sur le design des politiques publiques. Elle revient à poser la question de la gouvernance de ces sociétés marquées par des classes sociales moyennes émergentes. Dans cette perspective, il s'agit entre autre choses de savoir si les classes moyennes sont explicitement ciblées comme objectifs de politiques publiques spécifiques ou si elles n'en sont que des enjeux collatéraux.

L'analyse des liens entre classes moyennes et politiques publiques qui est proposée dans ce document est également historique et politique. Elle passe d'abord par la mise en perspective 
historique des liens entre les trajectoires de développement économique et les configurations de stratification sociale dans chaque pays, puis par l'interprétation systématique des documents produits par les autorités politiques (les plans émergences ou les documents stratégiques le plus souvent) pour comprendre quelles catégories de population sont visées et quelles sont les priorités arrêtées. Nous nous intéressons également de très près à l'ensemble des acteurs impactant ou étant impactés par l'émergence des classes intermédiaires de revenus (grands employeurs privés, banques, assurances, pourvoyeurs de services marchands d'éducation, de santé, de communication, associations de consommateurs, partis politiques, syndicats, etc.) et à leur rôle dans l'émergence de la perception politique et sociale du problème des classes moyennes dans les pays en développement. Pour cette raison, un second volet d'enquête qualitative a été consacré à des entretiens semi-directifs dits institutionnels avec les différents groupes d'acteurs intervenant soit dans la formulation, soit dans la mise en œuvre (en tant qu'offreurs de biens, de services ou de crédits) de politiques publiques sectorielles consacrées au moins pour partie aux classes moyennes. L'objectif de ces entretiens a été de voir si et comment l'expansion des classes moyennes affecte les agendas politiques et les grandes orientations des entreprises et des banques répondant à leurs attentes. Nous interrogeons, à travers ces entretiens institutionnels, l'influence des classes moyennes sur la définition des politiques publiques et le ciblage réel ou opportuniste des classes moyennes par les politiques publiques.

La confrontation des informations obtenues auprès des acteurs des politiques publiques et opérateurs des stratégies privées et auprès des ménages de la classe moyenne permet de vérifier la cohérence entre les aspirations des cibles de l'action publique et les offres politiques qui leur sont proposées.

\section{Les classes moyennes dans les économies en développement : problèmes de définition et d'identification}

\subsection{La définition des classes moyennes dans les pays en développement : un écart entre discours et réalité}

L'expression "classe moyenne" pose partout, au Nord comme au Sud, des problèmes méthodologiques considérables matérialisés par le jeu de mots "middle class, muddle class". La notion est en fait indissociable des trajectoires historiques nationales qui modèlent leur composition et leur dynamique particulières. Néanmoins, la notion de classe moyenne persiste à être utilisée car elle est englobante et permet de décrire et résumer "par évocation » certains enjeux économiques et politiques auxquels de nombreux pays en développement sont confrontés aujourd'hui. Le phénomène de transformation des stratifications sociales, de même que la somme des enjeux socio-politiques associés à ce changement économique, peuvent être vus comme un moment historique de la trajectoire de développement de nombreux pays en développement qui, depuis le début des années 1990, sont parvenus à réduire durablement l'incidence de la pauvreté. Dans ce contexte, le risque est grand que l'expression classes moyennes ait d'abord une vocation performative. La référence à cette notion suffit pour objectiver par le discours des changements 
complexes et difficiles à rapprocher, et à les faire accepter sous ce nom comme une réalité partagée par un grand nombre de pays en développement. Ce discours sur les classes moyennes se diffuse aux sphères expertes qui légitiment la notion et ses effets hypothétiques de sorte qu'il est ensuite repris systématiquement dans diverses littératures sans véritable effort de vérification.

Toutefois, la présente analyse comparative montre que le discours des "classes moyennes" ne va pas nécessairement de soi dans le contexte spécifiquement national des pays en développement, puisqu'il fait l'objet de vifs débats dans chacun des quatre pays étudiés. La tonalité, les positions et les objectifs de ces débats sont d'ailleurs très différents d'un pays à l'autre.

Au Brésil et en Turquie, par exemple, la mise en avant de l'expansion de la classe moyenne est un élément important du discours des autorités, même si les objectifs sont différents dans les deux pays.

Au Brésil, la notion de classe moyenne est largement portée par le discours officiel qui a contribué à sa médiatisation durant les années 2000, et ce jusqu'à la crise de 2014-2015, pour justifier l'efficacité des politiques mises en œuvre depuis la présidence de Lula ainsi que la position du Brésil au sein du club des BRICS. C'est dans cette atmosphère assez euphorique que le gouvernement, qui avait créé en juillet 2008 un Secrétariat des Affaires Stratégiques (Secretaria de Assuntos Estratégicos - $S A E$ ) avec rang de ministère, chargé d'aider le gouvernement à formuler des politiques publiques de long terme, l'a par la suite plus particulièrement orienté vers la définition et le suivi des actions en vue de consolider la classe moyenne. La « classe $\mathrm{C}$ » (sur un spectre de 5 « classes » allant de A à E), présentée comme composée désormais de près de $54 \%$ de la population brésilienne et disposant d'un pouvoir de consommation de 1000 milliards de Reais (SAE, 2012; 2013), représentait, dans l'esprit des gouvernants, le visage d'un nouveau Brésil, fier de ses progrès et démontrant l'efficacité des politiques sociales conduites depuis la présidence de Lula, à partir de 2003. Le Centre des Politiques Sociales de la Fondation Getúlio Vargas (CPS-FGV) a également joué un rôle éminent, sinon pionnier, dans les études portant sur la stratification de la société brésilienne.

En Turquie, l'AKP (Parti de la Justice et du Développement) au pouvoir soutient depuis presque dix ans, à la fois par ses politiques et ses discours, la formation d'une nouvelle classe moyenne anatolienne, religieuse et entrepreneuse au dépend de la vieille classe moyenne laïque issue du kémalisme. Les enjeux politiques relatifs à l'expansion de la taille de la classe moyenne apparaissent clairement dans le changement enregistré dans la définition des groupes à partir des indicateurs de statut socio-économique en 2012. En fondant sur le seul revenu de la personne qui assure le revenu principal (et non plus comme avant sur une combinaison d'un niveau de revenu, d'éducation et de détention de biens), le critère de classification des ménages, une augmentation artificielle de la taille des groupes intermédiaires a été obtenue permettant au pouvoir d'annoncer "l'enrichissement du peuple turc » entre 2006 et 2012. Dans le nouveau tableau des statuts socio-économiques, une personne qui gagne 5000 TL par mois (environ 2800 USD) est considérée comme relevant de ces groupes, même si elle est illettrée. Autrement dit, le pouvoir 
d'achat est pratiquement devenu le seul critère pris en compte, les critères relatifs à l'éducation ou au mode de vie ont perdu toute valeur.

Par contraste, la notion de classe moyenne est quasi absente des débats du quotidien ou des documents officiels en Côte d'Ivoire et au Vietnam, alors même qu'elle est au contraire très présente dans la presse économique et la presse généraliste ${ }^{11}$. Un buzz médiatique sur les classes moyennes est systématiquement entretenu par les acteurs économiques des marchés de la consommation et de la publicité dans ces deux pays. Dans la réalité, cependant, les changements sont beaucoup moins nets et font désormais ouvertement douter de la pertinence de l'utilisation du terme classe moyenne dans ces contextes, notamment en Afrique (Melber, 2016). Tout d'abord, ce qui, dans un pays comme la Côte d'Ivoire, est qualifié d'émergence des classes moyennes correspond à deux réalités différentes : (i) pour l'essentiel à un enrichissement régulier des catégories les plus aisées, de sorte que ce qui est qualifié de "classe moyenne" se polarise sur les ménages les plus riches de la population et (ii) des trajectoires de sortie de pauvreté, instables et variables dans leur durée et leur fréquence, sans toutefois que ces dynamiques soient suffisantes pour accroitre et stabiliser la base de la classe moyenne et confirmer son existence. L'identification à la classe moyenne dans ces deux pays n'apparaît guère non plus dans nos enquêtes qualitatives, les enquêtés au sein des ménages ne se décrivant pas machinalement comme familiers de cette catégorie mais se caractérisant comme étant "ni riches ni pauvres". Quant aux interlocuteurs rencontrés dans le cadre des entretiens institutionnels en Côte d'Ivoire, ces derniers définissent un périmètre de la classe moyenne en termes de revenu qui correspond au seuil d'entrée dans les 5\% les plus riches, alors qu'au Vietnam ils mobilisent des critères plus flous liés à la consommation ou au statut d'emploi. En fait, l'un des problèmes de la classe moyenne dans les pays encore globalement pauvres comme la Côte d'Ivoire et le Vietnam est précisément sa juxtaposition avec des taux élevés de pauvreté et une classe flottante très vulnérable qui exprime plus l'expansion de marchés du type Bottom of the Pyramid (Prahalad, 2009) que celle des classes moyennes « traditionnelles».

Plus globalement, la notion de classe correspond à une forme de stratification sociale bien spécifiée qui ne s'ajuste pas aisément à ce qui s'observe en République de Côte d'Ivoire, au Vietnam, et en partie seulement en Turquie et au Brésil. Dans aucun des quatre pays étudiés, la notion de classe moyenne ne semble pouvoir se ramener à une stratification sociale issue des conditions de production, comme ce fut le cas en Europe et aux Etats-Unis à partir de la seconde partie du 19ème siècle. Au Brésil, la classe moyenne est le produit des politiques de redistribution engagées depuis le début des années 2000 qui ont été facilitées par une configuration de croissance régulière à partir de la seconde moitié des années 1990. En Turquie, les conditions de fabrication d'une classe moyenne duale mettent également en œuvre d'autres principes de structuration que le positionnement objectif et subjectif dans les rapports de production. Au Vietnam, la classe moyenne est le produit à la fois des politiques anti-pauvreté, de deux décennies

11 Les documents officiels de planification stratégique comme le PND (2016-2020) en Côte d'Ivoire ou le SEDS (2011-2020) au Vietnam ne mentionnent pas les classes moyennes comme un objectif explicite des politiques publiques 
de croissance soutenue et du rejet des classes moyennes bourgeoises traditionnelles. Ses membres sont donc à la fois des employés, des travailleurs et de petits entrepreneurs indépendants, souvent du secteur informel, ou des agriculteurs ayant bénéficié des réformes agraires engagées dès la fin des années 1980. En outre, dans aucun des quatre pays, la mobilisation politique de ces classes moyennes ne s'est clairement manifestée à ce jour, ni sous forme de revendications directes organisées, ni sous celle de l'expression électorale. Il y a certes des différences significatives entre les quatre pays mais, mises à part des formes d'expression ponctuelles et qui ne se sont pas structurées politiquement au Brésil et en Turquie dans la période récente, rien ne se dévoile clairement ailleurs.

L'objectif de cette recherche est cohérent avec les réserves qui viennent d'être exposées puisqu'il vise à appréhender le phénomène "classes moyennes" dans les pays en développement, sans préalable idéologique ou théorique, notamment à travers l'identification de leurs caractéristiques socio-économiques, de leur dynamique de formation et de structuration et de leurs relations avec les politiques publiques. Cette approche permet d'éviter d'avoir à considérer les classes moyennes comme un idéaltype, ou un concept transféré de contextes historiques et socio-économiques différents. Elle permet plutôt de les concevoir comme un ensemble de caractéristiques constitutives d'un groupe hétérogène d'individus et de ménages toujours affectés par des formes de fluidité sociale et qui ne sont plus pauvres sans pour autant être riches.

\subsection{Identification monétaire et caractérisation statistique des classes moyennes}

\subsubsection{La délimitation des classes moyennes de revenu}

Dans la littérature économique, l'identification statistique de la classe moyenne repose la plupart du temps sur la définition d'un intervalle de revenu dans lequel se situent les individus ou ménages qui la composent. L'examen de la littérature récente sur les classes moyennes souligne l'absence de consensus dans le choix des intervalles de revenu adoptés. L'approche relative consiste à définir cette classe comme la population qui se situe au milieu de la distribution du revenu. Elle a le mérite d'adapter le critère d'identification au niveau de développement. Au sein de cette approche, la démarche la plus courante consiste à construire les intervalles à partir de fractions du revenu médian ou du revenu moyen : 75\%-125\% du revenu médian (Birdsall et al., 2000; Pressman, 2007), 50\%-150\% du revenu médian (Castellani et al., 2014), 60\%-200\% du revenu médian (Peichl et al., 2010) ou 100\%-250\% du revenu moyen (Song et al., 2015). Il est également possible d'adopter une définition basée sur les quintiles en définissant la classe moyenne comme les ménages ou individus appartenant aux trois quintiles du milieu de la distribution (Easterly, 2001 ; Castellani et Parent, 2011) ou au 3ème et 4ème quintiles (Alesina et Perotti, 1996).

L'approche absolue à partir de seuils internationaux exprimés en dollars PPA (parité des pouvoirs d'achat) est privilégiée lorsqu'il s'agit de définir des critères qui permettent la comparaison internationale. Alors que Milanovic et Yitzhaki (2002) proposaient de définir cette classe comme l'ensemble des ménages dont le revenu par tête se situe entre les revenus par tête moyens du 
Brésil et de l'Italie, soit entre 10 et 20 dollars par jour en PPA 2005, le seuil international de pauvreté de $2 \$$ s'est ensuite progressivement imposé comme borne inférieure de l'intervalle de revenu : $2 \$-10 \$$ pour Banerjee et Duflo (2008), $2 \$-13 \$$ pour Ravallion (2010) ou encore $2 \$-20 \$$ pour l'ADB (2010). La classe moyenne est alors supposée commencer là où la pauvreté se termine. Cette approche dominante suppose qu'il est suffisant d'être non pauvre pour être membre de la classe moyenne. Cependant, les ménages échappant à la pauvreté grâce à un revenu par tête situé entre $2 \$$ et $4 \$$ par jour restent fortement vulnérables à un retour dans la pauvreté en cas de chocs socio-économiques (ADB, 2010 ; Birdsall et al., 2014). Pour cette raison, ils peuvent être difficilement assimilés à une classe moyenne en stock, c'est-à-dire à des ménages stabilisés et au sens occidental du terme, c'est à dire comme des ménages capables de se projeter dans une trajectoire intergénérationnelle de promotion socio-économique. En réaction, une limite inférieure de $10 \$$, plus respectueuse de la définition précédente, a été utilisée : $10 \$-50$ pour Birdsall (2010) et Ferreira et al. (2013) ou 10\$-100\$ pour Kharas (2010). Certaines études adoptent également un seuil de 4 (Clément et Rougier, 2015).

Une troisième approche, dite approche mixte, consiste à combiner une borne inférieure absolue et une borne supérieure relative. A titre d'exemple, Birdsall (2010) propose de retenir une limite inférieure fixée à 10\$ (en PPA 2005) et une limite supérieure correspondant au 95ème percentile de la distribution du revenu. Selon Birdsall (2010), cette limite relative et donc spécifique aux pays permet d'isoler la part de la population dont le revenu est essentiellement composé de revenus du patrimoine et se différenciant donc du reste de la population par sa faible dépendance au travail. Cette approche mixte a été également été adoptée par Sosa Escudero et Petralia (2010) ou Bonnefond et al. (2015).

Le Tableau 1 compare les estimations de la taille de la classe moyenne pour les quatre pays telle qu'elle peut être estimée à partir de plusieurs intervalles monétaires différents appliqués aux données d'enquêtes ménages représentatives les plus récentes (voir page 7 pour le détail des sources utilisées). On obtient des estimations très disparates selon les critères de bornage, mais également entre les pays, puisque la taille de la classe moyenne de revenu varie entre $20-22 \%$ et $73-76 \%$ de la population totale pour le Brésil et la Turquie, contre un intervalle plus grand de $22,6 \%$ à $90 \%$ (pour le critère $2 \$-95$ ème percentile) de la population totale pour le Vietnam. Il est à noter que l'amplitude est également grande pour la Côte d'Ivoire où la part du groupe de revenu intermédiaire varie de 1,4\% (pour le critère 10\$-90ème percentile) à près de 46\%. Toutefois, l'utilisation de critères similaires aux trois autres pays définit une taille de la classe moyenne ivoirienne systématiquement plus faible. 
Tableau 1. Identification des classes moyennes de revenu.

\begin{tabular}{|c|c|c|c|c|c|c|}
\hline Pays & \multicolumn{2}{|c|}{$\begin{array}{l}\text { Proportions minimum et } \\
\text { maximum de la classe } \\
\text { moyenne avec différents } \\
\text { intervalles de revenu (\%) }\end{array}$} & $\begin{array}{l}\text { Intervalle de } \\
\text { revenu } \\
\text { sélectionné* }\end{array}$ & $\begin{array}{c}\text { Proportion } \\
\text { des pauvres } \\
(\%)\end{array}$ & \multirow[t]{2}{*}{$\begin{array}{c}\text { Proportion } \\
\text { de la classe } \\
\text { moyenne } \\
(\%) \\
614\end{array}$} & $\begin{array}{l}\text { Proportion } \\
\text { des riches } \\
(\%)\end{array}$ \\
\hline Brésil & 20,0 & 73,1 & [10\$; P95] & 33,6 & & 5,0 \\
\hline Côte & 1,4 & 45,9 & {$[4 \$ ; \mathrm{P} 95]$} & 68,6 & 26,4 & 5,0 \\
\hline d'Ivoire & & & & & & \\
\hline Turquie & 21,3 & 76,8 & [10\$ ; P95] & 21,6 & 75,4 & 5,0 \\
\hline Vietnam & 22,6 & 90,0 & {$[4 \$ ; P 95]$} & 22,5 & 72,5 & 5,0 \\
\hline
\end{tabular}

Source : auteurs.

* Les intervalles testés sont les suivants : $[2 \$ ; 10 \$] ;[2 \$ ; 20 \$] ;[\$ ; 20 \$] ;[10 \$ ; 20 \$ ;[10 \$ ; 50 \$] ;[10 \$$; $100 \$] ;[75 \% ; 125 \%$ revenu médian $] ;[50 \% ; 150 \%$ revenu médian $] ;[100 \% ; 250 \%$ revenu moyen $] ;[10 \$$; P90]; [10\$ ; P95].

Comme les distributions de revenus des ménages sont très différentes d'un groupe de pays considérés à l'autre (Brésil et Turquie vs Côte d'Ivoire et Vietnam), nous avons choisi de retenir dans la suite de l'analyse les critères mixtes (combinant une borne inférieure absolue et une borne supérieure relative) reportés dans le Tableau 1 (colonne 3) parmi l'ensemble des intervalles testés.

Comme nous l'indique le Tableau 2, alors que moins d'un demi-pourcent des ménages turcs vivent avec moins de 2 dollars par jour et par habitant, près de la moitié des ménages ivoiriens se trouvent dans cette situation. De même, alors qu'environ un tiers des ménages brésiliens vivent avec moins de 10 dollars par jour et par habitant, cette situation concerne plus des deux-tiers des ménages vietnamiens. On voit bien qu'en matière d'identification de la pauvreté, ici selon un critère absolu, les réalités locales sont difficilement conciliables et comparables.

Tableau 2. Caractéristiques des distributions de revenu et seuils de pauvreté par pays.

\begin{tabular}{lcccccc}
\hline Pays & $\begin{array}{c}\text { Ménages au revenu par tête } \\
\text { quotidien en dollars PPA } \\
(\%)\end{array}$ & $\begin{array}{c}\text { Revenu par } \\
\text { tête moyen en } \\
\text { dollars PPA } \\
\text { par jour }\end{array}$ & $\begin{array}{c}\text { Revenu par tête } \\
\text { médian en } \\
\text { dollars PPA par } \\
\text { jour }\end{array}$ & $\begin{array}{c}\text { Seuil de pauvreté } \\
\text { relative à } 60 \% \mathrm{du} \\
\text { revenu médian en } \\
\text { dollars PPA par } \\
\text { jour }\end{array}$ \\
\hline Brésil & 3,1 & 8,4 & 33,6 & 23,5 & 13,9 & 8,3 \\
Côte & 49,1 & 68,6 & 88,3 & 5,2 & 2,1 & 1,3 \\
d'Ivoire & & & & & & 18,6 \\
Turquie & 0,2 & 2,7 & 21,6 & 25,1 & 7,3 & 11,2 \\
Vietnam & 5,0 & 22,5 & 67,4 & 9,6 & 7,3 & 4,4 \\
\hline
\end{tabular}

Source : auteurs. 
Dans une perspective comparative, il nous faut pourtant établir des intervalles de revenu au sein desquels se situent les classes intermédiaires faisant l'objet de notre étude. Le choix de la borne droite des intervalles retenus ne pose pas de problème du fait de sa relativité ; nous excluons dans chaque pays les $5 \%$ de ménages les plus riches qui n'appartiennent pas à la classe de revenu intermédiaire. Par contre, dans le cadre de ce travail, il est impossible de choisir une borne gauche, borne absolue, commune à l'ensemble des pays étudiés. En effet, choisir par exemple une limite inférieure de la classe intermédiaire de revenu de 4 dollars PPA par jour et par tête permet bien d'exclure les ménages les plus pauvres de la distribution des revenus au Vietnam par exemple, mais inclut alors dans les « revenus intermédiaires » des ménages brésiliens ou turcs qui avec un revenu compris entre 4 et 10 dollars sont en situation de pauvreté au regard de leurs standards locaux. Le choix d'une borne inférieure à 10 dollars serait ainsi plus approprié pour la Turquie ou le Brésil, mais il exclurait une grande part de ménages faisant localement partie des ménages à revenu intermédiaire au Vietnam et a fortiori en Côte d'Ivoire. Choisir une borne inférieure relative commune à tous les pays ne permet en aucun cas de résoudre ce problème de comparabilité entre les pays. Si l'on choisit par exemple d'exclure des classes intermédiaires de revenu les 10\% de ménages aux revenus les plus faibles, on voit bien dans le Tableau 2 qu'il restera alors énormément de ménages (très) pauvres dans l'intervalle considéré en Côte d'Ivoire ou encore au Vietnam. Si l'on élève ce seuil à $25 \%$ par exemple, en écartant le quart des ménages les plus pauvres, on aura encore énormément de ménages pauvres dans la classe intermédiaire ivoirienne et on aura simultanément perdu un nombre considérable de ménages turcs à revenu intermédiaire ( $>10$ dollars), dorénavant considérés comme pauvres.

A notre sens, le groupe à revenu intermédiaire est composé de ménages qui ne sont ni pauvres, ni parmi les 5\% les plus riches. Ce «ni pauvres » décrit toutefois une réalité différente selon les pays considérés et nécessite donc des seuils différents selon les cas. Partant des seuils bas communément utilisés dans la littérature sur les classes moyennes (4 et 10 dollars PPA), nous avons pour chaque pays retenu celui qui s'approchait le plus d'une approche relative et donc locale de la pauvreté, correspondant à $60 \%$ du revenu médian (voir Tableau 2). Ainsi, c'est le seuil de 10 dollars qui a été choisi pour la Turquie et le Brésil dont les seuils de pauvreté relative, tels que décrits précédemment, s'établissent respectivement à 11,2 et 8,3 dollars PPA. Pour la Côte d'Ivoire et le Vietnam, nous avons retenu le seuil de 4 dollars PPA, pour des seuils de pauvreté relative respectifs de 1,3 et 4,4 dollars PPA. On notera que la Côte d'Ivoire fait ici vraiment office de cas à part puisque le niveau de pauvreté y est tel qu'il est bien possible qu'un seuil à 4 dollars puisse exclure des classes de revenu intermédiaire un certain nombre de ménages pourtant sortis des formes les plus dures de la pauvreté au regard des standards locaux. C'est ici toute la question de la signification de la floating class (revenus situés entre 2 et 4 dollars PPA) dans la compréhension de la croissance des classes moyennes qui est posée. 


\section{Figure 1. Distributions des revenus des ménages appartenant à la classe de revenu intermédiaire (kennel density function).}

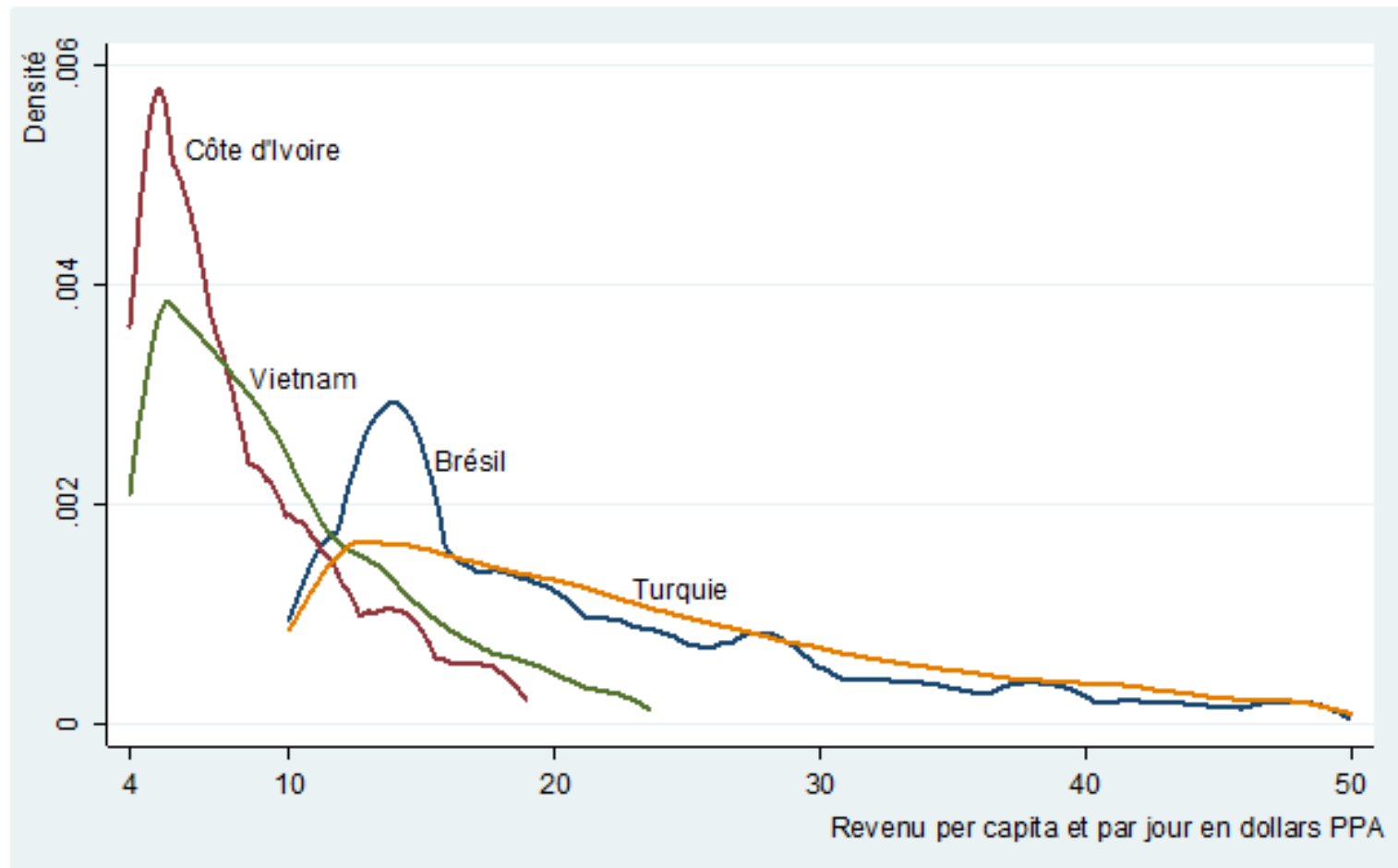

Source : auteurs.

Les classes intermédiaires de revenu ainsi définies comptent alors 26,4\% des ménages pour la Côte d'Ivoire, 61,4\% pour le Brésil, 72,5\% pour le Vietnam et 75,4\% pour la Turquie. Le « volume » de la classe intermédiaire de revenu est donc vraiment beaucoup plus faible en Côte d'Ivoire qu'au Vietnam et est plus important en Turquie qu'au Brésil. La Figure 1 présente la distribution des revenus par tête et par jour des ménages appartenant à la classe intermédiaire de revenu telle qu'établie précédemment. On constate que les ménages de cette classe se concentrent fortement au bas de l'intervalle de revenu en Côte d'Ivoire et dans une moindre mesure au Vietnam. Ceci pourrait signifier que l'essentiel des ménages considérés sont plus « tout juste sortis » de la pauvreté que réellement «installés » dans un statut de classe moyenne. Les distributions brésilienne et turque sont beaucoup moins asymétriques.

\subsubsection{L'bétérogénéité des classes moyennes nationales}

L'identification de la classe moyenne à partir d'un intervalle de revenu permet donc les comparaisons internationales. Pourtant, le choix de l'intervalle conditionne fortement la taille de cette strate intermédiaire de revenu. De plus, le groupe est identifié sur la base d'un critère de revenu qui gomme l'hétérogénéité propre à tout groupe social. Or, comme les pauvres ou les riches, les classes moyennes forment des groupes hétérogènes du point de vue des marqueurs plus sociologiques des classes sociales comme la consommation, le type d'emploi ou l'éducation. La prise en compte de cette hétérogénéité est le principal point d'ancrage de la littérature 
sociologique sur la stratification sociale, qui propose de définir la classe moyenne sur la base du statut professionnel et de l'éducation. La littérature économique sur les classes moyennes dans les pays en développement a toutefois commencé à articuler les critères économique et sociologique dans une approche intégrée qui permet, comme dans Bonnefond et al. (2015), Song et al. (2015) ou Andrianampiarivo (2016), d'identifier des groupes socio-économiques homogènes au sein de la strate de revenu intermédiaire.

Tableau 3. Synthèse des typologies des classes moyennes.

\begin{tabular}{|c|c|c|c|c|}
\hline & Côte d'Ivoire & Vietnam & $\begin{array}{c}\text { Brésil } \\
\end{array}$ & Turquie \\
\hline $\begin{array}{l}\text { Retraités et } \\
\text { inactifs }\end{array}$ & $\begin{array}{l}15 \% \\
\text { Moins éduqués, davantage } \\
\text { de femmes chefs de ménage, } \\
\text { revenus moyens, faible } \\
\text { équipement, faible } \\
\text { connectivité, faible demande } \\
\text { de loisirs }\end{array}$ & $\begin{array}{l}14 \% \\
\text { Urbains, moins éduqués, } \\
\text { revenus plus faibles }\end{array}$ & $\begin{array}{l}\text { 30\% } \\
\text { Ruraux, moins éduqués, } \\
\text { revenu plus faibles, faible } \\
\text { taux d'équipement, faible } \\
\text { connectivité } \\
2 \% \\
\text { Actifs (travailleurs non } \\
\text { rémunérés dans l'agriculture } \\
\text { de subsistance) }\end{array}$ & $\begin{array}{l}39 \% \\
\text { Moins éduqués, davantage } \\
\text { de femmes chefs de ménage, } \\
\text { revenus moyens, faible taux } \\
\text { d'équipement, faible } \\
\text { connectivité, faible demande } \\
\text { de loisirs }\end{array}$ \\
\hline Agriculteurs & $\begin{array}{c}\mathbf{2 5 \%} \\
\text { Agriculteurs ou salariés } \\
\text { agricoles informels, faibles } \\
\text { éducation et revenus, faible } \\
\text { équipement, faible } \\
\text { connectivitè, faible demande } \\
\text { de loisirs }\end{array}$ & $\begin{array}{l}\text { 27\% } \\
\text { Faibles éducation et revenus, } \\
\text { plus âgés, mono-activité, plus } \\
\text { âgés }\end{array}$ & Non pertinent & $\begin{array}{c}15 \% \\
\text { Agriculteurs ou salariés } \\
\text { agricoles informels, faibles } \\
\text { éducation et revenus, faible } \\
\text { taux d'équipement, faible } \\
\text { connectivité, faible demande } \\
\text { de loisirs }\end{array}$ \\
\hline $\begin{array}{l}\text { Travailleurs } \\
\text { informels }\end{array}$ & $\begin{array}{c}39 \% \\
\text { Tous secteurs d'activité, } \\
\text { faibles éducation et revenus, } \\
\text { jeunes, migrants }\end{array}$ & $\begin{array}{l}\text { 36\% } \\
\text { Urbains, industrie, secteur } \\
\text { privé, formels et informels, } \\
\text { travailleurs non rémunérés, } \\
\text { faibles éducation et revenus }\end{array}$ & $\begin{array}{c}7 \% \\
\text { Tous secteurs d'activité, } \\
\text { faibles éducation et revenus }\end{array}$ & Non pertinent \\
\hline $\begin{array}{l}\text { Travailleurs et } \\
\text { employés du } \\
\text { secteur formel }\end{array}$ & $\begin{array}{c}4 \% \\
\text { Urbains (Abidjan), éducation } \\
\text { et revenus élevés }\end{array}$ & $\begin{array}{l}16 \% \\
\text { Urbains, salariés, employeurs } \\
\text { et indépendants, formels et } \\
\text { informels, services, revenus } \\
\text { élevés }\end{array}$ & $\begin{array}{l}\text { 20\% } \\
\text { Urbains, services, éducation } \\
\text { élevée, faibles revenus } \\
\qquad \mathbf{2 4 \%} \\
\text { Agriculture et industrie, } \\
\text { faibles éducation et revenus }\end{array}$ & $\begin{array}{c}\text { 31\% } \\
\text { Tous secteurs d'activité } \\
\text { « traditionnels », emplois } \\
\text { salariés formels et petits } \\
\text { patrons urbains, éducation } \\
\text { secondaire, faibles revenus }\end{array}$ \\
\hline $\begin{array}{l}\text { Employeurs, } \\
\text { managers et } \\
\text { cadres et } \\
\text { professions } \\
\text { intermédiaires }\end{array}$ & $\begin{array}{c}1 \mathbf{1 7 \%} \\
\text { Urbains, secteur public, } \\
\text { éducation et revenus les plus } \\
\text { élevés }\end{array}$ & $\begin{array}{c}\mathbf{7 \%} \\
\text { Urbains, secteur public, } \\
\text { éducation et revenus les plus } \\
\text { élevês }\end{array}$ & $\begin{array}{c}\mathbf{1 7 \%} \\
\text { Urbains, secteurs privé et } \\
\text { public, éducation et revenus } \\
\text { les plus élevés, fort taux } \\
\text { d'équipement, pluriactivité }\end{array}$ & $\begin{array}{c}17 \% \\
\text { Services, secteurs privé et } \\
\text { public « modernes } \%, \\
\text { éducation et revenus les plus } \\
\text { élevés, forte demande de } \\
\text { loisirs, forte connectivité }\end{array}$ \\
\hline $\begin{array}{l}\% \text { de précaires } \\
\% \text { d'informels }\end{array}$ & $\begin{array}{c}79 \% \\
\text { Près de } 60 \%\end{array}$ & $\begin{array}{c}77 \% \\
\text { Au moins 50\% }\end{array}$ & $\begin{array}{c}\text { Entre } 39 \% \text { et } 83 \% \\
\text { Moins de } 10 \%\end{array}$ & $\begin{array}{c}44 \% \\
\text { Faible } \\
\end{array}$ \\
\hline
\end{tabular}

Source : auteurs

Pour les ménages appartenant à la catégorie intermédiaire de revenu délimitée dans l'étape précédente, la procédure de classification décrite dans l'Encadré 2 a été mise en œuvre pour chaque pays à partir des variables ayant trait à l'éducation (niveau d'éducation le plus élevé atteint par le chef de ménage) et l'emploi (catégorie socioprofessionnelle, statut dans l'emploi, type d'employeur ou secteur institutionnel et pluriactivité du chef de ménage) qui sont documentées dans les enquêtes ménages. Cette procédure a permis de mettre en évidence un degré important de fragmentation des classes moyennes nationales, puisque le nombre statistiquement robuste de sous-groupes cohérents identifié est de quatre pour la Turquie, cinq pour la Côte d'Ivoire, six 
pour le Vietnam et sept pour le Brésil. Le Tableau 3 récapitule et juxtapose les sous-groupes de la classe moyenne des quatre pays à des fins de comparaison. Il permet de mettre en lumière à la fois des spécificités nationales et des traits communs transnationaux dans la structuration de la classe moyenne.

Le Tableau 3 met en évidence que, dans les quatre pays de notre échantillon, la classe moyenne de revenu est très fortement fragmentée. Pour le Brésil, l'analyse quantitative met en évidence la forte fragmentation de la classe moyenne avec pas moins de sept sous-groupes identifiés : (i) les retraités et inactifs $(30 \%)$; (ii) les professions intermédiaires et fonctionnaires (7\%) ; (iii) les employeurs et cadres $(10 \%)$; (iv) les retraités "actifs" $(2 \%)$; (v) les travailleurs des secteurs primaire et secondaire (24\%); (vi) les travailleurs du secteur tertiaire formel (20\%) et (vii) la classe moyenne "informelle" (7\%). La structure socio-économique du groupe intermédiaire de revenu de la Turquie est plus homogène puisque seulement quatre sous-groupes ont pu être identifiés : (i) les retraités et inactifs (39\%); (ii) les exploitants agricoles $(13 \%)$; (iii) les travailleurs du secondaire et du tertiaire formel et les « petits patrons » $(31 \%)$ et (iv) les employeurs et cadres (17\%). En Côte d'Ivoire la classe moyenne est composée de cinq sous-groupes distincts : (i) les agriculteurs (25\%); (ii) les travailleurs de l'économie informelle (39\%); (iii) la classe moyenne "intermédiaire" du secteur privé formel (4\%); (iv) les dirigeants, cadres et professions intermédiaires du secteur public (17\%) et (v) les retraités et inactifs (15\%). Au Vietnam, la classe moyenne est composée de six sous-groupes : (i) la classe moyenne des retraités et inactifs (14\%); (ii) la classe moyenne des dirigeants, cadres et professions intermédiaires du secteur public $(7 \%)$; (iii) la classe moyenne des indépendants, employés et employeurs des services (16\%); (iv) la classe moyenne des employés de l'informel $(30 \%)$; (v) la classe moyenne des travailleurs non rémunérés $(7 \%)$ et (vi) la classe moyenne des exploitants agricoles $(27 \%)$.

Sur la base de la comparaison de ces classifications, un certain nombre de lignes de démarcation socio-économiques communes aux quatre pays analysés ont pu être identifiées. La Figure 2 cidessus en synthétise schématiquement quelques principaux aspects en illustrant les différences entre les groupes composant la classe moyenne des quatre pays considérés du point de vue de leur niveau moyen de revenu, de leur degré d'informalité dans l'emploi et du poids du salariat en leur sein. 
Figure 2. L'hétérogénéité des classes moyennes dans les 4 pays étudiés

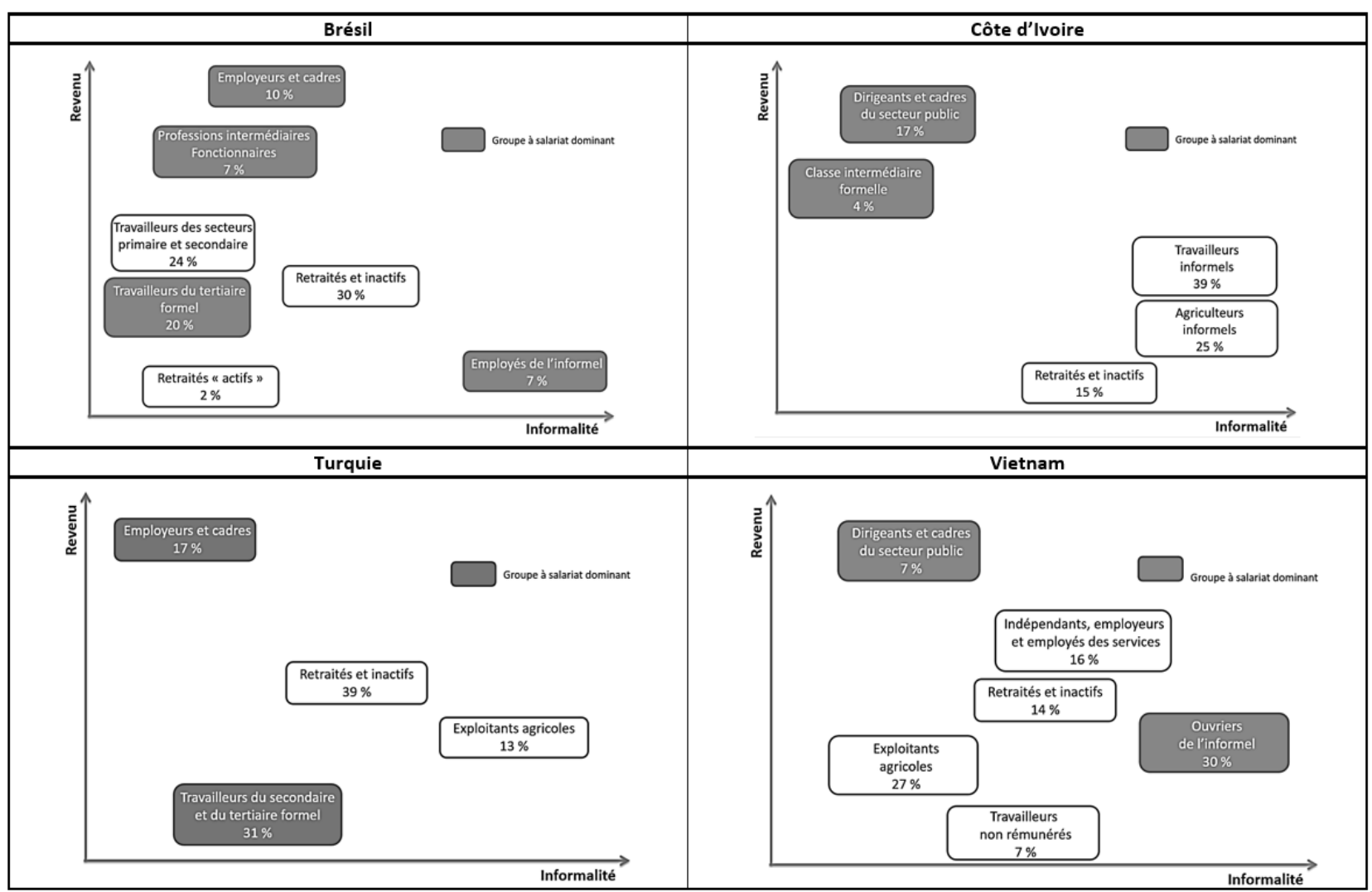

Source : auteurs

\subsection{Traits communs de la structuration socio-économique des classes moyennes nationales}

\subsubsection{L'absence de classe moyenne "moyenne"?}

Un premier trait commun est la polarisation de la classe moyenne sur une strate haute de taille limitée et une strate basse massive. La combinaison entre éducation élevée, stabilité de l'emploi et occupation (formelle) différencie le haut du groupe des revenus intermédiaires de sa strate basse dans les quatre pays. Bien que cette différenciation socio-économique semble commune aux quatre pays, la taille du groupe de revenu intermédiaire combinant des hauts niveaux d'éducation, une stabilité de l'emploi et accédant à des consommations modernes (loisirs, connectivité, biens durables) augmente avec le niveau de développement du pays.

Au Brésil, par exemple, les membres des sous-groupes des professions intermédiaires et fonctionnaires et des employeurs et cadres représentant 17\% du groupe de revenu intermédiaire se distinguent nettement des autres en présentant des niveaux d'éducation supérieurs, en occupant des emplois stables (salariat ou entreprenariat) dans des CSP élevées et en disposant de revenus nettement supérieurs. Ces deux sous-groupes composent probablement la classe 
moyenne "haute" et regroupent l'essentiel des fonctionnaires (même si des salariés du privé et des entrepreneurs composent également ces groupes). Les ménages composant cette frange supérieure de la classe moyenne se distinguent également des autres groupes par leur meilleur équipement en biens durables et par leur propension à davantage scolariser leurs enfants dans des établissements privés. Les cinq autres sous-groupes (représentant 83\% de la classe moyenne) renvoient à des réalités très diversifiées et regroupent des individus dont les revenus, les niveaux d'éducation et la qualité/stabilité de l'emploi (CSP inférieures, informel, agriculteurs) sont significativement plus faibles, et qui constituent les composantes basses et vulnérables de la classe moyenne. Si l'on ajoute un autre sous-groupe correspondant aux salariés intermédiaires du formel privé et public, dotés de niveaux de revenu et d'éducation intermédiaires et représentant $20 \%$ de la classe moyenne, on obtient respectivement un ensemble de $37 \%$ du groupe de revenu intermédiaire disposant de caractéristiques proches des "classes moyennes" au sens des pays industrialisés : relativement éduqués, fiscalisés et non vulnérables de par le statut de leur emploi et la régularité de leurs salaires ${ }^{12}$. Avec une classe moyenne haute représentant $48 \%$ du groupe de revenu intermédiaire, la Turquie présente des profils de structuration du groupe de revenu intermédiaire assez proches du Brésil, ce qui était prévisible compte tenu de la proximité de leur niveau de développement. Comme on pouvait également l'anticiper, dans ces deux pays le poids du segment supérieur de la classe moyenne (urbain, revenu moyen, éducation moyenne à supérieure, statuts d'emploi sécurisés) dans l'ensemble des ménages du groupe de revenu intermédiaire représente environ le double de celui observé en Côte d'Ivoire (21\%) et au Vietnam $(23 \%)$.

En Côte d'Ivoire, le dualisme de la classe moyenne est particulièrement marqué. La strate haute de la classe moyenne est constituée des dirigeants, cadres et professions intermédiaires du secteur public et de la classe moyenne intermédiaire du secteur privé formel. Cette strate représente 21\% de l'ensemble de la classe moyenne ivoirienne, et sa composante issue du secteur privé seulement 4\%. Elle semble constituée d'héritiers des groupes intermédiaires des années 1960-1970 qui, en dépit de 30 années de crises et d'instabilité, ont bénéficié d'un transfert intergénérationnel sur les différents types de capitaux nécessaires au succès (humain, économique et financier, relationnel et culturel). Ses modes de vies s'apparentent plus facilement à ceux des catégories aisées, à la fois sur le plan du confort matériel que sur le plan des pratiques de consommation (fréquentation plus régulière des supermarchés et malls) et d'investissement (dans l'immobilier ou les plantations, comme l'hévéa). A côté de cette strate haute, un processus d'émergence de "néo classes moyennes" par le bas (la petite prospérité) semble également être à l'œuvre. Dans ce processus, on va retrouver le groupe le plus important de la classe moyenne ivoirienne, celui des travailleurs de l'économie informelle urbaine (39\%), ainsi que le groupe des agriculteurs (25\%). Au-delà de leur revenu moyen bas, ces deux groupes ont en commun leur forte vulnérabilité (instabilité des revenus, insécurité foncière, etc.). Leurs conditions de vie restent assez précaires et confirment l'idée de ménages en flux incessants entre vulnérabilité et enrichissement tendanciel à la marge.

12 Le Brésil compte en outre $24 \%$ de travailleurs formels et informels dans l'agriculture et l'industrie qui pourraient constituer un groupe de réserve pour cette classe moyenne haute et intermédiaire, mais dont les potentialités de mobilité sociale ascendante restent limitées par des niveaux d'éducation relativement bas et des statuts d'emploi non sécurisants. 
$\mathrm{Au}$ Vietnam également, la classe moyenne est fortement marquée par le dualisme. Une strate haute, limitée en taille, est constituée de deux sous-groupes représentant respectivement 7 et $16 \%$ de la classe moyenne vietnamienne : les dirigeants, cadres et professions intermédiaires du secteur public (qui sont à plus de 80\% des employés du secteur public) très éduqués et dont les revenus sont les plus élevés de la classe moyenne, et les indépendants, employés et employeurs des services, qui sont essentiellement urbains, plutôt bien éduqués $(60 \%$ ont une éducation secondaire), et disposent de revenus relativement élevés par rapport aux autres. Ces deux sousgroupes sont les plus proches de la notion classique de la classe moyenne, même si une partie des ménages du second groupe est liée à l'informel et est plus vulnérable car ne bénéficiant pas de l'assurance sociale. Une large strate basse est composée des retraités et inactifs (14\%) au niveau d'éducation et de revenu relativement plus faible que celui des autres sous-groupes, et des employés de l'informel (30\%) majoritairement constitué d'ouvriers, disposant d'un niveau d'éducation moyen, que l'on retrouve dans le secteur manufacturier, de la construction et des transports où ils sont généralement employés par des unités de production privées exerçant au sein de l'économie informelle. Si l'on ajoute les travailleurs ruraux non rémunérés (7\%) qui rassemblent des chefs de ménage travailleurs non rémunérés exerçant dans leur grande majorité en milieu rural dans l'agriculture formelle, et le sous-groupe des exploitants agricoles $(27 \%)$ constitué d'agriculteurs (ainsi que des pêcheurs et des exploitants forestiers) indépendants formels assez peu éduqués et aux faibles revenus, la strate basse et vulnérable représente plus de $75 \%$ de l'ensemble de la classe moyenne vietnamienne. Une spécificité vietnamienne est que, quelle que soit la strate de la classe moyenne (haute ou basse), une partie des chefs de ménages combinent une activité agricole à leur activité salariée ou indépendante.

\subsubsection{Des classes moyennes de salariés plus que d'entrepreneurs?}

Une particularité commune aux quatre pays est qu'il n'existe pas de groupe de classe moyenne d'entrepreneurs, contrairement à certaines préconceptions de cette catégorie. Dans les quatre pays, les entrepreneurs se distribuent dans plusieurs groupes selon les caractéristiques de leurs entreprises ou du secteur d'activité. Ils ont donc des caractéristiques très différentes d'un groupe à l'autre et d'un pays à l'autre. En d'autres termes et contrairement aux hypothèses théoriques se basant sur les faits stylisés inspirés par les trajectoires de développement industriel européenne et nord-américaine et le rôle qu'y ont joué les entrepreneurs (Doepke and Zilibotti, 2008), l'entreprenariat n'est pas un réel facteur de différenciation au sein de la classe moyenne dans les pays en développement de notre échantillon.

Cela ne signifie évidemment pas qu'il n'existe pas d'entrepreneurs au sein des classes moyennes des économies en développement. Les capacités d'épargne et d'endettement peuvent être utilisées par une petite partie des groupes à revenu intermédiaire pour structurer des activités de marché, créer des entreprises formelles et investir dans la productivité. Mais, comme le montrent Banerjee et Duflo (2008), il s'agit d'un entreprenariat souvent informel, contraint financièrement, peu productif et peu pourvoyeur d'emploi formel. Pour la plupart des ménages de la classe moyenne qui possèdent une entreprise, cette dernière est juste une source de revenu supplémentaire et les investissements productifs sont moins importants que les investissements en capital humain. 
En Côte d'Ivoire comme au Vietnam, on retrouve ces entrepreneurs informels dans la strate basse de la classe moyenne (quand la strate haute est composée de salariés). Il est difficile de se prononcer catégoriquement sur la nature spécifique des dynamiques entrepreneuriales ici à l'œuvre, tant le secteur informel est hétérogène et recouvre des situations allant de l'entrepreneuriat de subsistance à l'entrepreneuriat d'opportunité. Si nos résultats témoignent d'une certaine précarité de leurs conditions de vie, ces indépendants et leurs microentreprises se situent à des niveaux de revenus qui les éloignent pourtant assez nettement du seuil de pauvreté. Il n'est donc pas impossible que ce sous-groupe des entrepreneurs informels recouvre pour partie celle des "constrained gazelles" identifiées par Grimm et al. (2012). On peut en effet aisément considérer que le sens de l'entrepreneuriat de cette catégorie des classes moyennes est bien présent, ne serait-ce que dans sa capacité à jouer de la pluriactivité dans une logique d'accumulation extensive, mais aussi dans les comportements d'épargne et d'investissement, dans la prise de risque, la recherche du profit et la volonté de transmission d'un patrimoine économique. Seulement, ce comportement entrepreneurial s'exprime dans un environnement particulièrement instable et contraint. Les entretiens qualitatifs menés en Côte d'Ivoire ont par ailleurs mis en évidence des aspirations réelles au devenir entrepreneurial pour les jeunes des milieux urbains comme ruraux (ce qui traduit bien sûr aussi la faiblesse des opportunités d'emplois formels).

En définitive, la Turquie est probablement le seul pays où un groupe concentre l'essentiel des entrepreneurs. Ce sous-groupe correspond à la nouvelle classe moyenne anatolienne et religieuse soutenant le régime de l'AKP. La croissance du secteur manufacturier axé sur les exportations a été un élément décisif de la croissance des petites et moyennes entreprises privées basées en Anatolie. Dans ce processus, des villes comme Kayseri, Konya, Denizli, Gaziantep, Kahramanmaraş, Malatya, Afyon, Çorum et Eskisehir sont apparues comme des « histoires de réussite » et la croissance et le succès des petites et moyennes entreprises sont allés de pair avec l'expansion des classes moyennes en Anatolie.

Banerjee et Duflo (2008) ont montré que, plus que le développement d'activités entrepreneuriales, c'est le fait d'occuper un emploi salarié stable et la régularité des revenus associés qui est un marqueur fort d'appartenance à la classe moyenne la moins vulnérable et qui distingue fondamentalement la classe moyenne des pauvres. Alors que les travailleurs temporaires ou précaires n'ont pas de sécurité sur les salaires, le temps de travail ou les contrats, les travailleurs permanents qui composent généralement la classe moyenne profitent d'une stabilité de leur revenu et peuvent accumuler des compétences dans l'emploi qui augmenteront leur productivité et leurs revenus futurs.

Au Brésil et en Turquie, cette thèse est largement vérifiée puisque les composantes hautes de la classe moyenne regroupent principalement des individus occupant des emplois stables (essentiellement salariés) dans des CSP supérieures ou intermédiaires. Au Brésil, l'enquête qualitative auprès des ménages a souligné que c'est plus par leur capacité à accéder à l'emploi formel, public ou privé, et à sécuriser cet accès pour leurs enfants, que par leur propension à prendre des risques et à créer des entreprises, que les ménages ont pu accéder à cette partie de la 
classe moyenne et s'y maintenir dans le temps. En Turquie, plus des deux tiers des chefs de ménages actifs de la classe moyenne exercent sous statut d'employé et/ou salarié. La relative sécurisation des revenus liée au salariat fonde largement la classe moyenne sur toute l'échelle des revenus considérés et dans tous les secteurs d'activité, aussi bien "traditionnels" (industrie, construction, transport, commerce et restauration) que plus "modernes" (information, communication, finance, immobilier). En Côte d'Ivoire et au Vietnam, la thèse de Banerjee et Duflo (2008) serait globalement confirmée si l'on réduisait la classe moyenne à ses composantes de la strate haute dont le revenu stable ou stabilisé reflète bien la situation de salariés du secteur officiel. Mais, comme l'avons vu plus haut, ce statut ne concerne qu'une minorité de la classe moyenne de revenu dans ces deux pays (autour de 12\% de la classe moyenne en Côte d'Ivoire et $15 \%$ au Vietnam) alors que 50\% et 90\% de l'emploi relève de l'informel. Dans ce large groupe, de nombreux chefs de ménages sont de petits entrepreneurs contraints du secteur des services et de l'agriculture, comme le montrent les données vietnamiennes.

\subsubsection{Informels, retraités et agriculteurs : des composantes atypiques de la classe moyenne?}

Dans chaque pays, l'analyse a permis de mettre en évidence l'existence d'une classe moyenne “informelle". Alors qu'au Brésil son poids reste très limité (seulement 7\% de l'ensemble de la classe moyenne), des parts beaucoup plus fortes sont observées pour la Côte d'Ivoire (39\%) et le Vietnam (30\%). En Turquie, le groupe des exploitants agricoles est à plus de $80 \%$ informel au sens du non enregistrement auprès des institutions de sécurité sociale. Le poids de ce groupe représente tout de même près de $13 \%$ du groupe de revenu intermédiaire. Ainsi, s'il est possible d'accéder aux catégories intermédiaires de revenu par le secteur informel, le poids du groupe des travailleurs ou entrepreneurs informels dans la classe moyenne nationale, c'est-à-dire de cette partie de la classe moyenne la plus vulnérable et largement en dehors des systèmes fiscaux, diminue avec le niveau de développement du pays.

Fait intéressant, dans chacun des quatre pays, un sous-groupe de retraités est identifié au sein de la classe moyenne. Sa taille, ses caractéristiques socio-économiques et le niveau moyen de revenu de ce groupe dépendent de nombreux facteurs comme le degré de maturité démographique du pays, la taille du secteur public ou l'existence de systèmes de retraite (généralement pour les anciens agents publics). Dans la plupart des pays de l'échantillon, ce sous-groupe correspond aux niveaux de revenus les plus bas de l'ensemble de la classe moyenne (sauf en Turquie où ils se situent au milieu de la distribution des revenus). Cependant, les caractéristiques de ce sous-groupe varient significativement d'un pays à l'autre. Son poids relatif dans le groupe de revenu intermédiaire est au moins deux fois plus faible dans les pays les moins développés (15\%) que dans les pays plus riches où il atteint 30\% au Brésil et 39\% en Turquie.

Au Brésil, la procédure de classification n'a pas permis d'identifier une classe moyenne spécifiquement composée d'agriculteurs. En revanche, une telle classe existe dans les trois autres pays, avec des tailles relatives deux fois plus grandes au Vietnam et en Côte d'Ivoire (près de $30 \%$ ) qu'en Turquie (13\%). En Côte d'Ivoire, l'importance du secteur agricole dans la formation de la classe moyenne ne doit pas surprendre, ce secteur représentant autour de $28 \%$ du PIB et 
constituant toujours un élément majeur du régime de croissance. Comme dans les deux autres pays, il est à noter qu'en plus des agriculteurs propriétaires de leurs terres, un certain nombre d'employés agricoles s'ajoutent à ces classes moyennes rurales, avec généralement des caractéristiques traduisant une très forte vulnérabilité (faibles revenus, faible éducation, pluriactivité).

Malgré l'apparence de caractéristiques communes dans les quatre pays, les différences en termes de revenus, de taille, de niveaux de vulnérabilité et de perspectives ou de capacités d'organisation collective, sont très marqués entre ces sous-groupes. Si certains auteurs voient ces sous-groupes de la strate basse de la classe moyenne (travailleurs de l'informel, agriculteurs, retraités et inactifs) comme une "catalyst class" ayant le plus d'intérêt en faveur de réformes significatives (Wietzke and Sumner, 2014), nos résultats soulignent leur grande hétérogénéité interne et leur faible capacité de mobilisation. En effet, il est important de noter que ces trois groupes ont, dans les quatre pays, des niveaux d'éducation plus bas que ceux des autres groupes, ce qui devrait limiter leur capacité à influencer les politiques publiques faute de capacités d'organisation et de coordination suffisantes ${ }^{13}$.

\subsection{Des trajectoires de stratification sociale fortement marquées par l'histoire}

L'analyse comparative des trajectoires de stratification sociale des quatre pays a mis en évidence l'historicité et la spécificité, dans chacun d'entre eux, de certains groupes sociaux, qui paraissent être très stables. Cette analyse souligne à la fois l'existence d'invariants dans leur structuration et la spécificité nationale de certains groupes, le tout étant le reflet du processus historique de stratification sociale associé aux transformations des régimes de croissance.

Le Brésil a longtemps été connu pour opposer une élite très réduite et aisée à une masse pauvre ou misérable et pour ne disposer que d'une classe intermédiaire restreinte (contrairement à l'Argentine). Néanmoins, une classe moyenne haute, urbaine et moderne, issue des sphères élevées de l'administration publique et des entreprises privées proches du pouvoir et composée de hauts cadres administratifs et commerciaux, d'ingénieurs ou de techniciens supérieurs s'est progressivement constituée et a survécu aux ajustements macroéconomiques des années 1990. La surreprésentation des fonctionnaires dans les strates supérieures de la classe moyenne mise en évidence par notre typologie témoigne de cet héritage historique. Cette classe moyenne historique se différencie désormais d'une nouvelle classe moyenne, vulnérable et très hétérogène du point de vue de ses caractéristiques socio-économiques, produite par le virage inclusif et redistributif des politiques publiques des années 2000. Ce n'est ainsi que relativement récemment que le poids de la classe intermédiaire de revenu s'est accru à un rythme soutenu comme corollaire de la

13 Le potentiel politique et économique d'une classe d'entrepreneurs agricoles peut toutefois être important si les conditions institutionnelles, économiques et technologiques sont propices à une progression rapide de la productivité et de la diversification agricole, comme l'a montré le précédent historique de Taiwan après les réformes foncières des années 1950 qui permirent à une classe de fermiers capitalistes de s'épanouir et de poser les bases de l'industrialisation qui allait suivre (The Economist, 2017). 
diminution de la pauvreté. Le contexte économique favorable (croissance économique soutenue, maitrise de l'inflation suite au plan Real de 1994 et maintien d'un système généralisé d'indexation du salaire minimum et de l'ensemble des allocations, pensions et bourses) a permis l'élévation des revenus réels. S'est ajoutée à cela la mise en œuvre de politiques sociales ambitieuses sous la présidence de Lula, notamment avec l'emblématique Bolsa Familia. Ce sont donc des millions de familles qui ont pu s'extraire de la pauvreté et sont venues gonfler les strates de revenu intermédiaire.

En Turquie, deux groupes de classes moyennes s'opposent dans leurs caractéristiques socioéconomiques ainsi que dans leurs orientations politiques. Les classes de retraités et inactifs et d'employeurs et cadres s'apparentent, au moins en grande partie, aux " anciennes classes moyennes» turques majoritairement issues des CSP supérieures. Elles disposent de revenus moyens plus élevés et d'un niveau d'éducation globalement supérieur, et occupent très majoritairement, pour leur part active hors-retraités, l'ensemble des secteurs d'activité "modernes" (information, communication, finance, assurance, immobilier, activités scientifiques et techniques) et des secteurs d'activité publics (administration publique, défense, éducation et santé). Par contraste, les classes d'agriculteurs, d'employés et de petits patrons s'identifient assez nettement aux nouvelles classes moyennes émergeant depuis le début des années 2000 en milieu urbain (travailleurs du secondaire et du tertiaire formel, "petits patrons ») comme en milieu rural (exploitants agricoles). Ces nouvelles strates ne correspondent pas forcément au standard des «anciennes» classes moyennes ; la partie urbaine, issue de CSP plus modestes, est globalement moins éduquée, dispose de revenus relativement faibles par rapport à la moyenne du groupe intermédiaire de revenu, et s'emploie très majoritairement dans les secteurs traditionnels de l'industrie, de la construction, du commerce, du transport ou encore de l'hôtellerie-restauration. La partie rurale de ces nouvelles classes moyennes est de son côté presque entièrement constituée d'exploitants agricoles très «traditionnels » (très peu éduqués, activité informelle, propriétaires de leur logement, peu de biens durables, pas de loisirs réguliers ni d'accès à internet), dont la promotion au sein des classes moyennes résulte probablement de l'ouverture de l'économie turque par les exportations agricoles et manufacturières. Les anciennes classes moyennes semblent plus installées dans le temps et dans la société urbaine, du moins dans les très grandes villes, mais elles sont en perte de vitesse dans le nouveau contexte politique de la Turquie. Un objectif explicite du régime de l'AKP est de faire monter en puissance la nouvelle classe moyenne anatolienne et religieuse dans la structuration socio-économique et politique du pays, aux dépens de la vieille classe moyenne. Historiquement, les classes moyennes turques furent d'abord urbaines, laïques, très éduquées et attachées aux idéaux du kémalisme. L'avènement du multipartisme dans les années 1950, la période de réformes libérales des années 1990 et l'arrivée au pouvoir de l'AKP ont cependant progressivement entraîné un changement dans la structure de classes. Les anciennes classes moyennes et les fonctionnaires ont peu à peu perdu du terrain au profit de nouveaux groupes sociaux qui ont profité des réformes économiques pour stabiliser leur situation et affirmer leur double volonté d'accumuler du capital et de perpétuer des valeurs plutôt traditionnelles. 
En Côte d'Ivoire, la formation des catégories intermédiaires au lendemain de l'indépendance s'est inscrite dans le sillage des processus d'enrichissement de la bourgeoisie d'Etat, en bénéficiant de l'expansion de l'appareil d'Etat et des activités économiques essentiellement liées à la rente agricole et à sa valorisation (Fauré et Médard, 1982 ; Contamin et Fauré, 1990). Dans cette république des "bons élèves", ces groupes intermédiaires ont appuyé leur enrichissement sur le rendement de leur investissement éducatif, en captant les emplois salariés les plus attractifs du public et du privé, mais aussi sur le rendement de leurs investissements fonciers. Jusqu'à la fin des années 1980, le groupe des agriculteurs (en particulier les planteurs) et celui des professions intermédiaires et des élites de l'administration publique (notamment dans l'éducation) forment ainsi l'essentiel des classes moyennes du pays. Les années 1990 marquées par un accroissement des inégalités consacrent la "séparation irréversible" entre ces classes moyennes et l'élite dominante (Vidal, 1997). La succession et l'accumulation de crises économiques, politiques et militaires qui s'ensuivirent jusqu'en 2010 a conduit à une paupérisation globale de la société ivoirienne avec un PIB par habitant retombant à un niveau proche de celui des années 1960 (Cogneau et al., 2016). Depuis le retour de la stabilité politique et de la croissance économique après la crise post-électorale de 2010-2011, les catégories moyennes peinent à retrouver une certaine prospérité comme en témoignent leur poids limité dans la population et la faiblesse de leur revenu moyen. La structure complexe de la classe moyenne ivoirienne traduit des continuités historiques avec des enjeux renouvelés dans le contexte des ambitions d'émergence de la Côte d'Ivoire. Les strates hautes composées des salariés du secteur moderne public et privé traduisent pour partie la permanence d'un stock qui se reproduit de manière intergénérationnelle (héritier des classes moyennes des années 1960-1980). La présence des agriculteurs s’inscrit dans la continuité historique d'une catégorie importante et protégée de la classe moyenne ivoirienne jusqu'à la fin des années 1980 qui subit aujourd'hui de plein fouet l'instabilité des prix internationaux des matières premières (cacao notamment) et l'insécurité foncière. Les entretiens ont révélé à quel point ces agriculteurs se sentent aujourd'hui abandonnés par les pouvoirs publics. Enfin, le poids considérable des informels urbains dans cette classe moyenne est à relier au redéploiement des actifs depuis les années 1980 dans les petites activités commerciales et industrielles pour échapper à la récession.

Au Vietnam, la trajectoire de formation de la strate de revenu intermédiaire est fortement marquée par l'histoire politique du pays des cinquante dernières années. Les classes moyennes et supérieures urbaines pré-socialistes, qui ont été privées de leurs possessions et de leur statut social par les politiques socialistes progressivement mises en place dans tout le pays entre les années 1950 et 1970, ont connu une mobilité sociale vers le bas justifiée politiquement par leur statut d'ennemis de classe du communisme. L'objectif du parti communiste Vietnamien était de mettre en place une société sans classe, et en particulier sans classe moyenne, assimilée par le régime à la petty bourgeoisie des systèmes capitalistes. Une première strate de classe moyenne a tout de même été reconstituée par la mise en place du système administratif et bureaucratique du régime socialiste qui a ouvert la possibilité de trajectoires de promotion sociale pour les enfants éduqués de nombreuses familles de rurales, comme en attestent les enquêtes qualitatives. À la suite des réformes de 1986 (Doi Moi) transférant progressivement le pouvoir économique en dehors de la bureaucratie du Parti (Beresford, 1993), cependant, des groupes sociaux nouveaux et diversifiés, y 
compris des propriétaires fonciers et une bourgeoisie urbaine naissante, ont commencé à réapparaittre dans les années 1990 sans encore complètement former une classe sociale (Pham Xuan Nam, 2002). Trois décennies après les réformes de rénovation du Doi Moi qui ont marqué le passage progressif de l'économie socialiste centralement planifiée à une économie mixte, de nouvelles couches moyennes de propriétaires fonciers, d'entrepreneurs et de travailleurs indépendants se sont agrégées aux groupes à revenu intermédiaire produits par le régime politique et économique et composé essentiellement de fonctionnaires actifs et retraités. Toutefois, ces différents groupes socio-professionnels ne constituent pas une classe moyenne partageant des valeurs communes. Elle n'est pas non plus identifiée comme telle par le pouvoir politique qui reste idéologiquement très frileux concernant ce groupe et qui continue à privilégier la réduction de la pauvreté et des inégalités sociales.

\subsection{Des classes moyennes vulnérables?}

Un premier résultat important de la première étape de classification et de caractérisation socioéconomique des différents groupes composant la classe moyenne du Brésil, de la Côte d'Ivoire, de la Turquie et du Vietnam est que ce qui correspond à la notion commune de "classe moyenne" peut être assez marginal dans les pays en développement, une grande partie de la classe moyenne étant très vulnérable du fait de la forte irrégularité et du faible niveau de ses revenus, de l'insécurité de son statut dans l'emploi et de son niveau d'éducation relativement faible.

Au regard de l'analyse comparative des typologies par pays, la part estimée des ménages vulnérables, travailleurs ou entrepreneurs informels, reste très élevée en Côte d'Ivoire (plus de 80\%), au Vietnam (plus de 50\%) et, de façon surprenante, en Turquie ${ }^{14}$ et au Brésil ${ }^{15}$. Cela confirme les hésitations constantes de la littérature à identifier les classes moyennes soit en termes de niveau de revenus ou de consommation, soit en termes de stabilité de la situation professionnelle et sociale. De fait, comme le montrent nombre de travaux (Banerjee et Duflo, 2008 ; Ravallion, 2010), une partie des catégories intermédiaires de revenu, la plus importante dans les pays émergents, est nourrie par des flux constants, ascendants et descendants, entre pauvreté et petite prospérité.

Nous pourrions ainsi utilement opposer : (i) un stock de classe moyenne, constitué des ménages aux statuts, rémunérations et niveaux d'éducation les plus élevés au sein de la classe moyenne, qui

14 Si l'on se réfère à l'approche du BIT définissant la vulnérabilité du travail sur la base des statuts dans l'emploi (travailleurs à compte propre et travailleurs familiaux), on peut considérer que plus de $16 \%$ des ménages de la classe moyenne sont vulnérables en Turquie.

15 Le pourcentage des ménages vulnérables est difficile à estimer au Brésil. Seuls 17\% des ménages de la classe moyenne (le groupe des cadres et employeurs et le groupe de professions intermédiaires) peuvent être considérés comme appartenant au segment supérieur de la classe moyenne. Les cinq autres composantes de la classe moyenne (donc $83 \%$ des ménages) présentent des niveaux de revenu moyen significativement plus faibles. Il est cependant important de préciser que parmi ces $83 \%$ de ménages, certains appartiennent à des groupes dont le statut occupationnel est relativement stable : travailleurs formels des secteurs primaire et secondaire (24\%) et travailleurs formels du secteur tertiaire $(20 \%)$. Si on considère que la stabilité de leur emploi les éloigne de la zone de fragilité, le poids des ménages vulnérables dans la classe moyenne se situerait à 39\%. 
n'en sortiront pas, sauf pour se hisser éventuellement au niveau supérieur de la distribution des revenus, et (ii) un flux constitué des ménages des parties basses de la classe moyenne qui ne cessent de lutter pour la conservation de leur mieux-être et ne peuvent stabiliser leur condition que difficilement et dans le long terme. Ces classes moyennes en flux sont instables dans leur composition, composées d'individus et de familles qui se sont extraits de la pauvreté mais ne présentent pas les caractéristiques socio-économiques (en termes d'emploi, d'éducation, de revenu, etc.) et de stabilité traditionnellement associées à la classe moyenne. Ces groupes que nous identifions dans les quatre pays sont proches de la notion de strugglers de Birdsall et al. (2014).

En Côte d'Ivoire, une partie significative de notre échantillon jouxte la catégorie de la classe dite "floating" par la Banque Africaine de Développement, ce qui suffit à souligner sa très grande vulnérabilité. La déstabilisation des catégories intermédiaires de revenu depuis les années 1980 et le caractère récent du retour de la croissance ne permettent pas de réduire une vulnérabilité extrême, ce qui se traduit par l'étroitesse de la classe moyenne dans le pays. L'essentiel de ces catégories basses de la classe moyenne sont donc en flux et ne possèdent pas les capitaux sociaux et économiques suffisant pour stabiliser leur situation et faire face aux chocs. Cela explique la quasi absence de référence à la classe moyenne dans nos entretiens qualitatifs. Au Brésil, de nombreux travaux ont souligné la vulnérabilité d'une partie importante de la classe moyenne qui a pu échapper à la pauvreté au cours des années 2000, grâce à la croissance économique, à l'augmentation du nombre d'emplois formels, aux politiques sociales mises en place sous l'ère Lula, et à l'élévation du revenu en termes réels, mais qui ne présentent pas les caractéristiques socio-économiques (en termes d'emploi, d'éducation, de revenu, etc.) et de stabilité traditionnellement associées à l'appartenance à la classe moyenne ${ }^{16}$. L'enquête qualitative a révélé que la fragilité de ces composantes de la classe moyenne risquait d'être exacerbée par la crise économique qui impacte le Brésil depuis 2014-2015 ${ }^{17}$. En Turquie, les « nouvelles classes moyennes » aux revenus relativement faibles apparaissent assez fragiles dans la mesure où, contraintes d'afficher leur appartenance à la classe moyenne par certaines formes de consommation ostentatoire, elles sont bien souvent identifiées comme les «classes endettées » ou comme les «classes moyennes à crédit». Une partie non négligeable des « anciennes classes moyennes » est également rendue vulnérable en cela qu'elle est régulièrement contestée sous le gouvernement de l'AKP depuis 2002 et que la promotion des «nouvelles classes moyennes » a tendance à se faire contre cette classe séculaire. Au Vietnam, enfin, un grand nombre d'entretiens de l'enquête qualitative souligne les contraintes de revenu auxquelles les ménages de la classe moyenne sont confrontés et la nécessité de la pluriactivité pour soutenir leur consommation. En

${ }^{16}$ Une étude de Porchmann (2012) montre que 94\% des emplois créés entre 2004 et 2010 correspondent à des postes faiblement rémunérés (jusqu'à 1,5 fois le salaire minimum), principalement créés dans les services, le commerce et dans les activités autonomes, sont de très faible qualification, assurés dans des conditions très précaires et soumis à une forte instabilité professionnelle.

${ }^{17}$ Le Brésil subit une crise économique sévère, marquée par un effondrement de la croissance économique (-3,8\% en 2015 et 2016) et dont le principal facteur déclencheur réside dans la baisse de la demande de la Chine (principal partenaire commercial du Brésil) pour les produits primaires et d'extraction. Cette crise s'est traduite par une recrudescence du chômage et de la pauvreté ainsi que par l'affirmation des problèmes de surendettement des ménages, autant d'évolutions qui pourraient impacter la classe moyenne brésilienne. 
revanche, l'endettement n'est pas signalé comme une stratégie d'accession à la classe moyenne, via la consommation ou les investissements, ni par les ménages, ni par les organisations interrogées dans les enquêtes qualitatives. Seul le motif de l'investissement productif est mentionné comme motivant l'endettement des entrepreneurs et indépendants financièrement contraints.

Au-delà de différences majeures de composition et de niveau de vulnérabilité, deux autres points communs ressortent de la comparaison des classes moyennes des quatre pays. Un premier point est que ces catégories se constituent pour la plupart sur la base d'une trajectoire intergénérationnelle qui permet de cumuler les capitaux de tous types (financier, culturel, social) et de stabiliser en partie dans le temps les effets de flux. Un second point est que ces catégories intermédiaires se constituent au moins en partie dans une relation conflictuelle avec l'Etat, leur émergence se faisant par des stratégies en marge des cadres étatiques, légaux et fiscaux. C'est particulièrement le cas des groupes de l'informel qui sont dans une relation complexe vis-à-vis du projet d'institutionnalisation de l'Etat.

D'après Birdsall et al. (2014), ce rapport conflictuel des composantes les plus vulnérables des catégories intermédiaires de revenu (i.e. les strugglers) s'exprime également à travers deux phénomènes. D’une part, au sein du système de redistribution, les strugglers sont des «payeurs nets ». En effet, ils bénéficient nettement moins des programmes de transferts publics que les plus pauvres et, dans le même temps, font face à une forte fiscalité indirecte. D'autre part, ne disposant pas du niveau de revenu des strates moyenne ou haute de la classe moyenne ou de la classe riche, les strugglers sont davantage dépendants des services publics de faible qualité, notamment dans le domaine de l'éducation, ce qui pourrait contraindre leurs perspectives de mobilité ascendante. Cette configuration est très forte en Amérique Latine, et elle a conduit à des troubles sociaux importants au Brésil et en Argentine ces dernières années. Elle pourrait également concerner un pays comme le Vietnam si le gouvernement réussit à formaliser une partie importante des entreprises et de l'emploi du secteur informel des services et de l'agriculture, c'est à dire s'il réussit à fiscaliser la partie de la classe moyenne qui repose assez largement sur les services publics d'éducation et de santé dont la qualité est faible, de l'aveu des ménages enquêtés. Pour l'heure, les contributeurs (et bien souvent les bénéficiaires à travers la protection sociale systématique) de la fiscalité vietnamienne correspondent aux $20 \%$ les plus riches, c'est à dire pour partie, le haut de la classe moyenne (OECD, 2014). En Côte d'Ivoire, les strugglers se situent pour l'heure assez largement en dehors des régimes fiscaux et du système formel de redistribution, mais ils restent néanmoins soumis à la fiscalité indirecte. Par ailleurs, nos résultats ont montré que ces derniers, et en particulier les informels urbains, sont ceux qui subissent le plus le poids de la pression redistributive communautaire (transferts de revenus entre ménages). Le positionnement de ces individus de la classe moyenne dans les réseaux de solidarité est en effet relativement asymétrique du fait de leur capacité à venir en aide aux membres les plus pauvres et de leur difficulté à accéder eux-mêmes à du soutien financier en cas de besoin (Kroeker, 2016). Au regard des défaillances actuelles des services publics qui leur sont potentiellement dédiés, leur intégration progressive aux systèmes formels de redistribution pourrait donc contribuer à renforcer leur sentiment d'être "corvéables à merci" pour reprendre 
l'expression d'un des interviewés et, de fait, conduire à des situations similaires à celles observées en Amérique Latine.

\section{Comportements et préférences des classes moyennes}

Nous proposons, sur la base des enquêtes qualitatives ménages, d'examiner les comportements, préférences et aspirations économiques, sociales et politiques révélés par les membres des classes moyennes dans les quatre pays de l'étude. L'objectif est double. Il s'agit d'une part d'identifier ces comportements et préférences, de les comparer entre classes moyennes nationales afin de savoir si ces groupes sociaux présentent dans ces domaines des spécificités. Sur la base de ces observations, il s'agit d'autre part de savoir s'il existe une communauté de comportements et de préférences homogènes qui permettrait d'attribuer une identité spécifique à ce groupe, susceptible notamment d'interpréter ses choix économiques et politiques.

\subsection{Consommation, éducation, santé, endettement et logement : une convergence des comportements socio-économiques des classes moyennes vers le mieux-être et la promotion sociale}

\subsubsection{Des hypothèses théoriques ...}

A la lumière d'un certain nombre de travaux récents, l'influence de l'expansion des classes moyennes semble opérer via deux canaux principaux : le niveau et la structure de la consommation et l'investissement dans le capital humain.

En théorie, la densification de la strate de population située à un niveau intermédiaire de l'échelle des revenus est un facteur d'industrialisation et de complexification de l'économie car ces ménages sont capables de consommer des biens de plus en plus diversifiés (Banerjee et Duflo, 2008) et d'occuper des postes de travail de plus en plus complexes et spécialisés. Du fait qu'elle présente un poids démographique supérieur à celui des groupes à revenu élevé, la classe moyenne peut notamment créer des opportunités d'économies d'échelle facilitant le développement d'activités industrielles (Murphy et al., 1989). De plus, l'accès des classes intermédiaires à la consommation de biens supérieurs (biens culturels, biens d'équipement) réduit progressivement leur prix, augmentant conséquemment l'accès des classes inférieures à ces consommations et libérant des ressources budgétaires pour les classes supérieures qui peuvent dès lors consommer de nouveaux produits et amorcer le développement de nouveaux secteurs (Matsuyama, 2002). Si la taille de la classe moyenne est trop limitée, ou si cette classe moyenne n'est pas suffisamment différenciée de la classe de revenu supérieur ou de la classe de revenu inférieur, alors le développement d'une production diversifiée est bloqué, et l'économie garde des caractéristiques dualistes, les plus riches consommant des biens de luxe importés, et les plus pauvres consommant des produits de première nécessité à faible productivité.

Le fait que des ménages pauvres accèdent en grand nombre à la classe moyenne devrait réduire l'épargne de précaution et augmenter la capacité d'épargne et d'investissement de l'économie. Les 
modèles de trappe de pauvreté ont montré que les inégalités d'accès au financement affectent les choix occupationnels et les investissements en capital humain des ménages des pays en développement, condamnant les plus pauvres au chômage ou à des emplois très peu productifs (Banerjee et Newman, 1993). Ce qui caractérise l'épargne des classes moyennes réside probablement davantage dans son utilisation que dans son niveau. L'émergence de la classe moyenne peut notamment durablement influencer les changements économiques à travers le canal de l'investissement dans l'éducation et la santé de la famille. En outre, Doepke et Zilibotti (2008) ont montré combien les classes moyennes, parce qu'elles participent de façon plus intensive à l'émergence de nouvelles activités que les autres groupes sociaux, tendent à investir plus dans le capital humain et à transmettre à leurs enfants des préférences pour l'éducation et l'effort, plutôt que des patrimoines financiers. L'émergence d'une classe moyenne éduquée et moins contrainte financièrement devrait, dès lors, favoriser le développement de l'emploi, de l'innovation et de l'entreprenariat, via les investissements réalisés dans des activités nouvelles.

\subsection{2. ... partiellement validées par les faits}

L'approche utilisée dans le cadre de ce projet permet de confronter ces prédictions théoriques à l'observation empirique des groupes de revenu intermédiaire de quatre pays aux niveaux de développement hétérogènes. Si les résultats obtenus dans le cadre des enquêtes qualitatives ne prétendent pas à être généralisables à l'ensemble des pays étudiés, et encore moins à l'ensemble des pays en développement, ils offrent néanmoins un riche matériau permettant de discuter les hypothèses précédentes. Si certaines dimensions caractéristiques des groupes à revenu intermédiaire sont communes aux quatre pays, comme la diversification de la consommation, l'investissement dans l'éducation privée et le recours au crédit, d'autres en revanche, comme les stratégies de résidence, sont spécifiques à un contexte national ou à un sous-groupe de la classe moyenne. De même, nous remarquons que si des comportements sont communs, leurs effets peuvent être très différents selon la structure particulière de chaque composante de la classe moyenne. Il est donc impossible de raisonner en globalité sur l'expansion de la classe moyenne et ses effets. Tout dépend de la structure de cet ensemble, des parties qui sont en expansion (les plus riches, les plus pauvres) et de l'intensification dans le temps de ces transformations.

\section{Un consumérisme soutenu par l'endettement}

Dans les quatre pays analysés, on observe une croissance rapide des consommations ostentatoires symboliques de la réussite et de la prospérité individuelle au sein des classes moyennes. Nouvelles technologies (smartphones, tablettes, etc.), loisirs (voyages, fréquentation accrue des parcs d'attraction, etc.) et achats d'équipements de transport individuel comme l'automobile en Turquie et au Brésil, ou les motocyclettes au Vietnam, sont devenus des marqueurs incontestables des modes de consommation de l'ensemble des classes moyennes et de leurs composantes. La Côte d'Ivoire reste un peu à l'écart de ce point de vue, du fait que ces consommations caractéristiques des classes moyennes ne concernent que le segment le plus élevé de la classe moyenne. 
Pour prétendre à ces consommations, une grande partie des classes moyennes des quatre pays doit se soumettre à des logiques d'endettement, ce qui entretient la vulnérabilité des ménages appartenant aux segments les plus bas du groupe intermédiaire. Au Brésil, par exemple, les prêts assortis de remboursements automatiques par prélèvements à la source sur les salaires (empréstimos consignados), rares jusque dans les années 2000, ont connu une forte expansion lors des mandats de la présidente Dilma Rousseff (2009-2016) qui furent caractérisés par l'explosion du crédit ${ }^{18}$. Pour la Turquie également, l'augmentation et la modification de la consommation des ménages des classes moyennes ont été rendues possibles par un endettement conséquent. La très forte corrélation entre l'utilisation des cartes de crédit et l'augmentation de la consommation des classes moyennes est la preuve que parallèlement à la progression des revenus, l'endettement a constitué un moteur essentiel du développement d'une certaine consommation ostentatoire de la classe moyenne turque au cours des années récentes. Au Vietnam, en revanche, la pluriactivité est préférée à l'endettement pour financer les nouvelles consommations du fait de la plus forte contrainte politique sur l'expansion du crédit privé que dans les autres pays de l'échantillon. En Côte d'Ivoire, dans un contexte de faible bancarisation des ménages et d'un faible financement des acteurs privés par le système bancaire, la diversification des activités est également le moyen d'un accroissement significatif des revenus pour soutenir la consommation.

Il convient de souligner que ces logiques d'endettement placent une bonne partie des classes moyennes des pays les plus développés, la Turquie et le Brésil, dans une situation de vulnérabilité particulière, formellement encouragée par les politiques publiques. Dans ces deux pays, les risques de mobilité sociale descendante sont importants pour une grande part du groupe de revenu intermédiaire en cas de resserrement du crédit. Au Brésil, l'emballement du crédit à la consommation et à l'équipement, ces dix dernières années, puis le retournement brutal de conjoncture, ont mis en grande difficulté de nombreuses familles. En 2005, seules 18,4\% de celles-ci étaient endettées. En septembre 2016, 58,2\% étaient dans ce cas. Un quart des familles endettées ont des difficultés de remboursement et, réglant leurs dettes avec retard, sont pénalisées par les taux d'intérêt pratiqués. Par ailleurs, en septembre 2016, 9,6\% des familles sont en situation d'insolvabilité (CNC, 2016). Il est également marquant de constater qu'en Turquie, les institutions interrogées s'accordent largement pour définir les classes moyennes comme des « classes endettées ». Pour des raisons différentes dans chacun des deux pays, le recours au crédit privé ne s'est pas généralisé en Côte d'Ivoire et au Vietnam où le risque de crise d'endettement est probablement moindre. Dans ces deux pays, le développement d'un secteur privé du crédit permettrait d'accroître l'accès aux consommations symboliques typiques des classes moyennes, mais pourrait également, à terme, superposer des problèmes de vulnérabilité financière à des problèmes de vulnérabilité économique pour l'importante strate basse de la classe moyenne.

18 La situation du Brésil est toutefois particulière et pas forcément représentative, car, si l'on se réfère à De la Torre et al. (2012), il apparaît que les pays disposant de caractéristiques proches de celles du Brésil ont un poids du crédit à la consommation deux fois plus faible que celui prévalant au Brésil, mais une part du crédit immobilier deux fois plus élevée. 
Notre approche qualitative permet tout de même de saisir un changement dans les objectifs assignés à la consommation selon les différentes composantes de la classe moyenne. A la suite de nos analyses quantitatives et qualitatives, trois types de ménages peuvent être distingués du point de vue du rapport aux consommations symboliques et à l'endettement. Les ménages les plus vulnérables du groupe de revenu intermédiaire, ceux qui sont peu différenciés des situations de précarité, témoignent de comportements consuméristes très contraints, essentiellement réalisés dans des espaces commerciaux locaux et consacrés principalement aux produits de première nécessité. L’accès au crédit est assuré via des circuits financiers de type microfinance, finance informelle ou plus récemment mobile money (cas de la Côte d'Ivoire notamment). Les ménages qui commencent à s'en sortir et s'éloignent du risque de retomber en pauvreté, dégagent des revenus disponibles plus importants et peuvent diversifier leurs consommations et les lieux de leur réalisation. Ils disposent d'une capacité nouvelle d'emprunt plus ou moins facilitée par les politiques de redistribution, comme c'est le cas au Brésil, en Turquie ou même au Vietnam grâce à des politiques très inclusives ou par le recours à la pluriactivité. Enfin, les ménages installés par leurs revenus ou par les capitaux constitués par leurs familles qui appartiennent depuis longtemps aux catégories de revenu intermédiaire et cherchent à maintenir leur statut en consommant les symboles et marqueurs de la classe moyenne soit par ostentation, soit par habitude. Pour ces catégories supérieures des classes moyennes, le crédit a une fonction différente puisqu'il est la condition de l'expansion économique via la constitution d'un patrimoine.

\section{L'expansion des dépenses privées d'éducation et de santé}

Comme Banerjee et Duflo (2008), nos résultats montrent que la classe moyenne poursuit par ses choix de dépenses et d'investissement une meilleure " qualité de vie ». Cette meilleure qualité de vie passe par une meilleure santé pour la famille et une éducation plus coûteuse, souvent dans le privé, pour les enfants. Ces dépenses sont des marqueurs importants de la classe moyenne dont elles traduisent les aspirations de promotion intergénérationnelle, comme en Turquie, au Vietnam et en Côte d'Ivoire, ou les efforts pour maintenir leur statut, comme au Brésil. Ces dépenses montrent aussi que les politiques publiques de ces pays sont focalisées sur les plus pauvres et laissent au marché le soin de s'occuper des autres composantes de la classe moyenne, quel que soit le pays, même ceux qui ont une histoire plus inclusive comme le Vietnam.

A travers notre analyse comparative, on retrouve ainsi un des faits stylisés majeurs présentés par les travaux d'économistes historiens (Doepke and Zilibotti, 2008) consacrés à l'émergence de nouveaux groupes sociaux au 19ème siècle, à savoir l'importance de l'éducation. Pour les ménages du groupe de revenu intermédiaire des quatre pays, investir dans la quantité et la qualité d'éducation pour soi et pour les enfants est présenté comme une stratégie prioritaire. Ceci se traduit par une forte propension des ménages de ce groupe à envoyer leurs enfants dans les écoles privées (Brésil, Turquie, Côte d'Ivoire) ou à investir dans des cours privés extrascolaires (Vietnam) pour améliorer la qualité des apprentissages. L'intérêt pour l'investissement dans le capital humain est clair dans les classes moyennes des quatre pays. Dans ces économies, l'aisance est traditionnellement identifiée à la détention d'un capital culturel sanctionné par un diplôme, chacun essayant d'obtenir le « graal » d'un emploi dans le secteur officiel public ou privé. 
Comme dans de nombreux pays à revenu intermédiaire, l'émergence d'une classe moyenne en Turquie s'est accompagnée d'un désengagement non négligeable de l'Etat qui a facilité l'expansion rapide de l'offre de marché à destination des classes moyennes et incité à la marchandisation de la demande des classes moyennes en matière d'éducation, mais également de santé. De surcroît, les politiques de l'AKP visant à favoriser l'accès à l'éducation privée des nouvelles classes moyennes, dirigées contre le segment des anciennes classes moyennes perçues comme privilégiées par les anciens régimes, ont été très bien reçues du fait de leurs visées explicitement "égalisatrices".

La situation au Brésil, au Vietnam et en Côte d'Ivoire est assez différente, mais les conséquences sont similaires sur les dépenses d'éducation. Au Brésil comme au Vietnam, les défaillances du système d'enseignement primaire et secondaire public sont largement reconnues, même si des efforts très importants ont été faits sur les dernières décennies pour améliorer l'accès de tous à l'éducation. Au Brésil, les établissements privés et payants sont de meilleure qualité pour les deux niveaux de scolarisation. Les familles de la classe moyenne, et notamment de la classe moyenne haute, privilégient la scolarisation de leurs enfants dans le secteur privé. Il s'agit indéniablement d'un marqueur fort d'appartenance à la classe moyenne au Brésil (voir plus haut, synthèse des résultats quantitatifs). La logique est la même dans le secteur de la santé avec un recours important aux plans de prévoyance privés. Au Vietnam, la faible qualité des écoles publiques à tous les niveaux est due à l'insuffisance de moyens humains d'un système scolaire qui a connu un développement rapide durant la dernière décennie. Les ménages de la classe moyenne sont dans l'ensemble conscients de ce défaut de qualité qu'ils cherchent à compenser en engageant des dépenses d'éducation privée sous la forme de cours particuliers après les journées de cours et sous la forme indirecte d'une augmentation du temps passé par les parents pour aider leurs enfants dans leurs devoirs. En Côte d'ivoire, les entretiens révèlent aussi un effort partagé par l'ensemble des catégories intermédiaires de revenus pour l'éducation des enfants qui apparait comme une de leur préoccupation majeure (conduisant à des sacrifices importants pour les composantes les plus vulnérables). Face au constat unanime des errements du système public d'éducation, les mêmes stratégies qu'au Vietnam sont mises en place et renforcées par l'expansion rapide de l'offre privée d'éducation secondaire et supérieure, via la création d'écoles et d'universités privées, qui viennent pallier la mauvaise qualité de l'enseignement public.

\section{Des stratégies spatiales et résidentielles de différenciation}

Le logement est une dimension des dépenses des ménages de la classe moyenne qui est importante car elle marque une différence avec les comportements des pauvres en la matière, notamment en Turquie et au Brésil. Dans ces deux pays, une conscience de classe s'exprime dans la recherche d'entre-soi qui caractérise les investissements immobiliers des ménages des classes moyennes.

En Turquie, les choix résidentiels ont toujours été l'un des principaux repères reflétant l'écart entre les différentes classes de la société. La périurbanisation et les gated communities sont devenues ces vingt dernières années l'un des caractères majeurs de l'urbanisation et de l'implantation 
géographique des classes moyennes. Parallèlement à la libéralisation de l'économie, cette frange de la société a connu à partir des années 1980 une évolution économique et sociale importante et décidé alors de s'installer dans des cités sécurisées en marge de la ville. Ce choix peut s'expliquer par une volonté de démontrer un certain statut, mais aussi par un désir de vivre loin du bruit et des embouteillages du centre-ville. La question du logement au Brésil est également directement reliée aux graves problèmes d'insécurité publique prévalant dans les villes brésiliennes. Dans ce contexte, les familles aisées et celles issues du haut de la classe moyenne ont tendance à se réfugier dans des ensembles immobiliers «bunkerisés » et gardiennés en permanence (condominios) entourés de murs et barbelés, et disposant de tous les conforts (piscines, salles de sport, salles de jeux et de fêtes, garages, etc.).

La situation est assez différente dans les deux autres pays de l'échantillon compte-tenu de la moindre qualité générale des logements et de la faiblesse des politiques publiques d'urbanisme et de logement. En Côte d'Ivoire, la question du logement du point de vue des ménages est marquée par la spécificité de la situation à Abidjan caractérisée par de fortes pressions inflationnistes dans un contexte de croissance de la demande et d'une offre qui peine à suivre. Ainsi, les ménages les plus vulnérables de la capitale ont plus de difficultés à satisfaire leurs besoins de confort et résident dans des quartiers excentrés pour bénéficier de loyers abordables quand les strates les plus hautes investissent dans l'immobilier pour diversifier leur revenu. En milieu rural, le prix du logement étant moins élevés, les revenus des classes moyennes agricoles permettent d'accéder plus facilement à la propriété. Au Vietnam, la plupart des ménages du groupe de revenu intermédiaire se plaignent de la vétusté de leur logement et souhaitent pouvoir investir plus pour améliorer la qualité et pour l'agrandissement de ce dernier. Dans ces deux pays, les stratégies immobilières sont moins guidées par la recherche d'entre-soi.

\subsection{Valeurs et engagement politiques des classes moyennes}

\subsubsection{Classes moyennes et démocratisation : une relation incertaine d'un point de vue théorique et empirique}

Nombre d'études antérieures transforment, par un simple procédé de requalification linguistique, un groupe de revenu en une classe sociale, c'est-à-dire en un acteur collectif produisant des actions collectives. Ce glissement sémantique conduit logiquement à attribuer aux classes moyennes des caractéristiques politiques hypothétiques fondées sur de simples postulats idéologiques, ce qui explique la grande incertitude des résultats obtenus. Or, les incertitudes quant à l'impact de la classe moyenne sur les évolutions économique et politique sont considérables.

Une partie de la théorie et certaines études empiriques prédisent que la classe moyenne serait un facteur important de changement politique car elle serait porteuse des valeurs qui sont essentielles à la promotion et à la consolidation démocratiques et parce qu'elle favoriserait la diminution de la conflictualité sociale. Les classes moyennes sont globalement associées à des performances économiques supérieures et permettraient la promotion d'une meilleure gouvernance et la diffusion des idées démocratiques (Easterly, 2001; ADB, 2010; Loayza et al. 2012). Elles 
joueraient un rôle de catalyseur au cours du processus de démocratisation (Lipset, 1959; Moore, 1966), réduisant l'intensité des conflits opposant une large classe de pauvres et une élite restreinte et pouvant faire obstacle au développement économique (Huntington, 1991). L'émergence d'un groupe intermédiaire permettrait d'atteindre des niveaux de taxation et de redistribution plus favorables à l'investissement et à la croissance économique, ce qui stabiliserait la démocratie en augmentant le coût d'opportunité de la répression politique pour les élites (Acemoglu et Robinson, 2006).

Inversement, d'autres études doutent de ces effets «logiques» et montrent que les classes moyennes ne sont pas mécaniquement porteuses de progrès économique et social. L'expansion des classes moyennes peut par exemple participer à l'arrivée au pouvoir des régimes non démocratiques, autoritaires voire totalitaires et contribuer à leur maintien tout en favorisant des modes de gestion fondés sur la corruption et le népotisme (Thompson, 2004; Chen et Lu, 2011 ; Villegas, 2012 ; Wiemann, 2015 ; Schotte, 2017). Certaines analyses critiques rappellent que les ménages appartenant à la catégorie de revenu intermédiaire sont hétérogènes du point de vue de leur situation sociale et économique, de leurs préférences socio-politiques et de leur acceptation de l'impôt, de sorte que leur mobilisation en tant que groupe sera quasi inexistante, et que leurs votes pas plus que leur préférence pour des styles de gouvernance, ne seront convergents. De plus, le rapport des classes moyennes au pouvoir politique est moins dicté par la nature du régime que par les garanties que donne celui-ci du maintien des conditions permettant l'enrichissement et l'accès à la promotion sociale. Tout régime assurant cette garantie, quelle que soit sa nature, obtient tendanciellement le soutien de ces classes intermédiaires et notamment des groupes en émergence sur une ou deux générations. L’histoire contemporaine présente des exemples répétés de ces configurations. Il existe notamment une littérature importante sur l'Asie qui montre que, en cohérence avec les valeurs traditionnelles asiatiques (les valeurs confucéennes par exemple) prônant le respect de l'ordre et de la hiérarchie, le travail et la discipline et souvent mises en avant par les autorités en place, les classes moyennes sont plutôt conservatrices et à l'arrière-garde de l'engagement politique (Hattori et Funatsu, 2003 ; Thompson, 2004). Enfin, ces groupes "classes moyennes", et notamment les plus influents, auront tendance, comme le rappellent Huntington (1968) ou Resnik (2015) à protéger avant tout leurs intérêts et à bloquer toute négociation favorable aux autres classes et notamment aux pauvres, quitte à entraîner la génération de troubles provoquant à leur tour une fermeture plus autoritaire du pouvoir.

L'influence réelle sur les changements institutionnels de ces classes moyennes va donc dépendre de leur capacité de mobilisation et de leur place dans les équilibres sociopolitiques structurant l'économie politique d'une nation (Acemoglu et Robinson, 2006). Or dans nos cas étudiés, leur capacité de mobilisation est faible (faute de cohérence interne) et leur poids global modeste (soit en termes démographiques par rapport aux pauvres, soit en termes qualitatif par rapport aux riches de la bourgeoisie d'état). 


\subsubsection{Un engagement politique très limité des classes moyennes nationales}

De manière générale, notre étude semble confirmer le faible degré d'engagement politique des classes moyennes. Les groupes de revenu intermédiaires sont très loin de constituer une classe sociale rassemblée autour d'une identité politique forte de classe ou d'intérêts agrégés. Cette tendance générale, qui s'exprime notamment par l'émiettement des aspirations et des formes de comportements politiques connait cependant des spécificités nationales claires, particulièrement marquées pour le Brésil contemporain.

En Turquie, notre étude met en évidence un mécanisme d'engagement politique très clientéliste. On peut ainsi affirmer que les nouvelles classes moyennes provinciales et métropolitaines et le segment inférieur des classes moyennes peuvent être placés au centre-droit du spectre idéologique, votant en majorité pour l'AKP qui subventionne ou construit des réseaux de patronage et ouvre des universités, des écoles privées et des marchés de consommation à l'adresse des ménages concernés. Les groupes de la classe moyenne et de la classe moyenne supérieure dotés d'un capital culturel élevé et laïques ont tendance à s'identifier au centre-gauche et à soutenir le CHP (Parti Républicain du Peuple). Alors que l'AKP bénéficie d'un large soutien en Anatolie, il est également fortement présent dans les grandes villes. Le CHP, pour sa part, est plus présent dans les grandes villes et les régions côtières. En somme, il semble que les classes moyennes, plutôt que d'instituer ou de porter le changement politique, votent de manière globale pour les organisations politiques qui garantissent et protègent au mieux leurs intérêts et ce quelles que soient leurs orientations politiques.

Les récents événements en Turquie ont montré la faible audience des aspirations des composantes les plus anciennes et des nouvelles classes moyennes stambouliotes sur les choix de politique publique de l'AKP orientés vers la diffusion d'un modèle consumériste à crédit et conservateur. La reprise en main autoritaire du régime et la répression de toute forme de contestation des politiques menées par le gouvernement ne devrait pas faciliter l'émergence d'une parole collective et unifiée des classes moyennes.

Au Vietnam, les ménages des classes moyennes sont accompagnés à titre individuel, puisque le gouvernement cherche à sécuriser les gains de bien-être provoqués par la croissance et les politiques d'inclusion sociale des plus pauvres à travers des politiques sectorielles dans l'éducation, les transports ou la libéralisation commerciale, mais pas à titre de collectif, c'est à dire en qualité de groupe social. Les classes moyennes Vietnamiennes ne semblent pas prêtes à se mobiliser collectivement contre cet état de fait, même si un embryon de contestation politique se fait entendre grâce à la diffusion des nouvelles technologies. En revanche, la corruption du système politico-économique est pointée du doigt par plusieurs membres des classes moyennes vietnamiennes interrogés dans l'enquête, mais cette critique reste diffuse et n'est pas relayée par des actions ou organisations structurées. L'engagement politique des membres de la classe moyenne reste donc limité et les préférences pro-démocratiques sont peu exprimées. En conséquence, l'impact politique de la classe moyenne sur les politiques publiques est aussi limité. Les couches moyennes de la population ne participent pas en tant que groupe aux intérêts 
clairement identifiés à la définition des politiques socio-économiques et ne sont pas non plus ciblées par des actions publiques sectorielles. Dans les enquêtes, les ménages s'accordent autour du fait que le gouvernement devrait consacrer plus d'investissements dans des domaines comme les infrastructures, l'éducation et la formation professionnelle qui renforceraient l'efficacité économique et la justice sociale et environnementale, tout en renforçant la communauté nationale. Enfin, les ménages manifestent une intolérance croissante à la corruption et au manque de transparence de la politique et de la bureaucratie. Pourtant, ils sont en même temps fortement réticents, pour des raisons qui ne sont pas clairement énoncées, à s'engager dans des activités collectives qui pourraient catalyser et exprimer leur mécontentement et revendications. La réponse politique à l'essor des classes moyennes s'avère insuffisante car elle reste centrée sur l'objectif d'inclusion des plus pauvres et ne perçoit pas clairement tous les enjeux de ce changement massif.

La même situation se retrouve en Côte d'Ivoire où elle s'illustre essentiellement par la quasiabsence de référence à ce thème au niveau des autorités publiques ou dans le discours des groupes de ménages. La forte hétérogénéité des groupes de revenu intermédiaires ne fait que confirmer que ce qu'on appelle classes moyennes correspond à une apposition de sous-groupes aux intérêts et configurations radicalement différents, poursuivant chacun des trajectoires différentes d'enrichissement et de sortie de pauvreté. Leur forte hétérogénéité interne et leur faible expérience de mobilisation collective réduisent leur capacité à faire pression sur le politique, sauf exceptions, notamment dans le cas de revendications de groupes intervenant alors plus sur la base de leur identité socioprofessionnelle (fonctionnaires et salariés du secteur privé formel par exemple, cas récent des militaires). L'engagement politique des classes moyennes ivoiriennes reflète ainsi très largement le constat global fait par l'OCDE sur l'ensemble de la population en 2013 : «en moyenne, 65\% des personnes interrogées se sentent indifférentes à la vie publique, un taux nettement supérieur à la moyenne des pays africains »(OCDE, 2016, p.148). Les résultats de nos entretiens ménages montrent aussi que ces classes moyennes sont essentiellement mues par la recherche d'intérêts personnels et assez matériels (conditions de vie, logement, sécurité, etc.) et la protection de leurs stratégies de promotion personnelle et familiale. Dans ce contexte, ces groupes hétéroclites et hétérogènes sont rarement en position de se mobiliser de manière unie et active pour faire pression sur le politique. Se font plutôt entendre des groupes qui interviennent plus sur la base de leur identité socio professionnelle (revendications salariales des fonctionnaires et notamment récemment des militaires par exemple). Pour autant, nos interviewés dénoncent de manière quasi unanime le creusement des inégalités sociales et la corruption. Ainsi, les mobilisations sociales qu'a connue la Côte d'Ivoire suite à l'augmentation du prix de l'électricité en 2015-2016 sont révélatrices de mouvements de contestations portés, notamment via les réseaux sociaux comme le "mouvement des 200", par des catégories de la population assimilables aux strates hautes de la classe moyenne ${ }^{19}$. La classe moyenne ivoirienne a la particularité d'avoir

19 En avril 2016 est créé le "mowvement des 200 », via une page Facebook et l'hashtag \#les200, porté notamment par Daouda Coulibaly, un journaliste et blogueur indépendant. Ce mouvement est né en réaction à une déclaration malheureuse du Ministre de l'économie numérique, qui avait assuré lors d'une interview que les revendications des internautes mécontents ne comptaient que 100 ou 200 personnes, car selon le Ministre, "seule une minorité pense que tout va mal en Côte d'Ivoire ». Ces "cyberactivistes», tels qu'ils se définissent, créent alors «la page des 200 personnes qui pensent que le pouvoir 
été particulièrement échaudée par les coûts de la crise politique et militaire de la décennie 2000, ce qui devrait favoriser dans l'ensemble une tendance au statu quo dès lors que le pouvoir garantit le retour à la stabilité et l'accès à la prospérité.

Parmi les quatre pays de l'étude, c'est probablement au Brésil que l'engagement politique des classes moyennes est le plus marqué, notamment sur la période récente. Le positionnement et l'engagement politique des classes moyennes sont fortement marqués par l'instabilité et la crise de confiance liée à la répétition des affaires de corruption d'Etat des derniers trimestres. Comme le reste de la population, la classe moyenne brésilienne exprime son rejet du système politique, quelle que soit l'échelle du pouvoir (fédéral, estadual ou municipal), et souligne son incapacité à œuvrer pour l'intérêt général. Elle porte en particulier un jugement très sévère sur la corruption endémique qui prévaut dans le pays. Dans le cadre de l'enquête qualitative ménages, les personnes interrogées ont ainsi mobilisé des qualificatifs très explicites pour décrire le monde politique ("horrible", "terrible", "corrompu", "immonde", etc.) et ont fait part de leur sentiment de révolte à l'encontre de ce système. Elles ont également souligné la réalité des petites corruptions quotidiennes dans les démarches administratives. Sur la période récente, les membres de la classe moyenne brésilienne, et notamment les plus vulnérables, ont pourtant témoigné de leur capacité à se mobiliser et manifester, comme ce fut notamment le cas lors des rassemblements massifs de mars à juin 2013. Le mouvement social a débuté par des manifestations de jeunes étudiants qui ont été rapidement rejoints par de nombreux enseignants, fonctionnaires, professionnels de santé, techniciens, cadres moyens et supérieurs du secteur privé et leurs familles, catégories socioprofessionnelles composant le cœur de la classe moyenne brésilienne. Si le message premier était une opposition aux dépenses faramineuses associées à l'organisation de la Coupe du Monde de football en 2014 et des Jeux Olympiques de Rio en 2016, les manifestations qui se sont déroulées au Brésil ont porté des revendications bien plus larges comme la dénonciation de la corruption et de l'insécurité publique ou des défaillances en matière de services publics.

Il convient d'ajouter que la classe moyenne est potentiellement menacée à court et moyen termes par les mesures actuelles du gouvernement pour faire face à la crise. Mais, les manifestations de 2013 ont montré que la classe moyenne a désormais le courage de revendiquer et que les classes moyennes peuvent se mobiliser collectivement autour de revendications partagées. Elles pourront peut-être infléchir des politiques publiques qu'elles jugent trop défavorables. De plus, bien qu'hétérogène, la classe moyenne brésilienne constitue un groupe de plus en plus informé et éduqué, capable de mieux spécifier, articuler et faire prévaloir ses demandes. Cela souligne le rôle clé de l'éducation dans le renforcement du rôle de la classe moyenne. Dans ce contexte, les classes moyennes brésiliennes portent à la fois des revendications de réformes universelles, comme les politiques de sécurité publique, de lutte contre la corruption et d'amélioration de la

d'achat des habitants de la Côte d'Ivoire devient de plus en plus faibles » et qui estiment que la population ne doit pas payer le lourd tribut des dettes accumulées et investissements de l'Etat. Ils affichent des slogans tels que : "Le pays est devenu joli mais \#les200 ont le ventre vide » ou "\#les200 ne veulent plus du cosmétique. Ils veulent $d u$ concret $»$. Très vite, le mouvement dépasse le simple enjeu de l'électricité pour embrasser d'autres revendications sociales (santé, matières premières, corruption, chômage, enjeux fonciers, etc.). 
qualité des services publics, et des réformes catégorielles comme le rééquilibrage la fiscalité et de la qualité des services publics à leur profit.

En définitive, autant les comportements économiques comme la consommation, l'endettement et l'investissement dans l'éducation semblent rapprocher les différentes classes moyennes nationales, autant les valeurs et les comportements politiques semblent les éloigner. Les rapports entre groupes sociaux sont fortement marqués par l'histoire politique de chaque pays. De plus, l'absence de valeurs politiques homogènes, et le faible engagement politique sont des caractéristiques partagées par les quatre pays qui tendent à remettre en cause la notion archétypale de classe moyenne actrice du changement politique et social. L'hétérogénéité du groupe, au cœur de cette situation, est matérialisée par l'opposition entre bas et haut de la classe moyenne, ou entre nouvelle et ancienne classe moyenne et se traduit comme en Turquie et en Côte d'Ivoire par des rapports au politique et au pouvoir très différents, les parties nouvelles étant plus prodémocratie et plus favorables à la protection des droits que les nouvelles. Reste que les classes moyennes soutiennent par leur inertie positive ou négative les régimes et gouvernements qui assurent leur prospérité et leur bien-être.

\section{Les classes moyennes et les politiques publiques}

Analyser comment les politiques publiques sont affectées par la densification des groupes de revenu intermédiaires revient à identifier (1) quelles sont les demandes politiques, économiques et sociales exprimées par la classe moyenne ou certaines de ses composantes, et (2) comment les gouvernements des pays en développement anticipent, ignorent ou, au contraire, répondent à ces demandes différentes. Cette cinquième section met d'abord en évidence que les demandes et aspirations exprimées par le groupe de revenu intermédiaire trouvent finalement peu de réponses en termes de politiques publiques spécifiquement dédiées à cette partie de la population. Elle montre ensuite que si du côté des gouvernements des quatre pays de notre échantillon, il n'y a pas de stratégie globale de promotion des classes moyennes, ces dernières, en dépit de leurs faibles capacités de mobilisation collective et de politisation, peuvent être instrumentalisées par le politique. Pour établir ces résultats, nous utiliserons les éléments tirés de la revue de littérature et des enquêtes qualitatives ménages et institutions conduites dans chacun des pays de l'échantillon.

\subsection{Des aspirations globalement insatisfaites par les politiques publiques}

La littérature économique et sociologique a identifié un certain nombre de marqueurs comportementaux propres aux classes sociales intermédiaires. Certains de ces marqueurs comportementaux ont été décrits dans la section précédente et convergent vers la volonté d'investir dans le capital humain, notamment l'éducation et la santé des enfants, afin d'échapper aux mécanismes intergénérationnels de transmission de la pauvreté. D'autres marqueurs sont propres à l'amélioration des conditions de vie et de l'environnement professionnel, notamment via la demande de logement et de transport. Ces comportements constatés traduisent autant d'aspirations et d'attentes de la part des classes moyennes sur différents secteurs de politiques 
publiques pour lesquelles les réponses apportées par les gouvernements en place semblent souvent inadaptées, partielles, inefficaces voire absentes.

\subsubsection{Des politiques d'accompagnement en matière de santé et d'éducation}

La santé et l'éducation sont deux secteurs sur lesquels les classes moyennes ont exprimé un certain nombre d'attentes dans les enquêtes. Ceci est assez conforme à la capacité des classes moyennes à se projeter dans l'avenir et à revendiquer une amélioration qualitative des conditions de vie.

Au Brésil, l'enquête qualitative “ménages” a révélé que c’est dans le domaine de la santé que les attentes sont les plus importantes. Pourtant, cette aspiration se heurte à l'absence de politique ambitieuse du côté de l'offre de politiques publiques, ce qui conduit les ménages de la classe moyenne à avoir massivement recours à des plans de prévoyance privés. Au Vietnam, les ménages du groupe de revenu intermédiaire aspirent à une meilleure qualité des soins et à une couverture plus universelle des risques de santé, notamment pour les travailleurs informels qui représentent au moins la moitié du groupe. Le gouvernement vietnamien a mis en place à la fin des années 2000 un plan ambitieux de protection sociale pour les individus les plus vulnérables, notamment les enfants, qui est venu compléter un régime préexistant s'adressant aux fonctionnaires, sans toutefois ouvrir ce droit aux très nombreux travailleurs du secteur privé qui ne disposent que d'un dispositif volontaire dont le taux de couverture est très faible. On observe une dynamique similaire en Côte d'Ivoire. La plupart des personnes interrogées dénoncent la qualité des structures de santé publiques (accueil, temps d'attente) et se tournent vers des structures privées lorsqu'elles en ont les moyens pour souscrire à une assurance maladie. Si les ménages interrogés estiment que le projet de Couverture Maladie Universelle du Président Ouattara est en soi un très bon projet, et se disent globalement prêts à contribuer à hauteur de 1000 FCFA mensuels par tête, ils demeurent circonspects sur sa mise en œuvre effective qui tarde à venir. En Turquie, l'un des principaux projets de l'AKP qui a directement affecté les classes moyennes lors de ces quinze dernières années a été la réforme du domaine de la santé. Le modèle de l'accès aux services de santé était très hiérarchisé jusqu'à la mise en place du «Programme de Transformation en Santé » (Sağlıkta Dönüşüm Programı) en 2003. En bref, avec cette réforme, il s'agissait d'abolir la distinction entre l'assurance maladie privilégiée des fonctionnaires et l'assurance générale des « autres », pour établir une " Assurance de santé générale ». Après la réforme, les hôpitaux publics « de qualité » se sont ouverts à toutes les catégories, ce qui a inévitablement perturbé les "privilégiés" de l'ancien système qui ont alors massivement recouru aux hôpitaux privés, comme pour se sentir privilégiés à nouveau. La réforme a ainsi provoqué une forte privatisation des services de santé et une libéralisation des conditions de travail des personnels de santé qui ont fortement affecté les classes moyennes.

En ce qui concerne l'éducation, les mêmes logiques s'observent, avec une aspiration forte à une éducation de qualité qui se transforme en dépenses d'éducation privée. 
En Turquie, l'éducation est un des postes de dépenses les plus différenciés entre les groupes sociaux. L'impératif éducatif - reposant sur la croyance encore très ancrée qu'un diplôme de l'enseignement supérieur est la meilleure garantie contre le chômage et pour l'ascension sociale oriente les stratégies démographiques (question de la taille idéale de la famille) et résidentielles des familles, quelles que soient les affiliations idéologiques. L'éducation est le domaine auquel les classes moyennes sont les plus sensibles, mais également celui qu'elles influencent le plus. Cause nationale, l'éducation est un chantier auquel les grands groupes qui dominent l'économie sont sommés de participer de multiples manières. Parallèlement, l'Etat a multiplié les initiatives pour que le secteur privé prenne en charge directement une partie de l'offre scolaire, de l'école primaire à l'université. De plus, les dépenses des familles pour l'éducation primaire de leurs enfants ont considérablement augmenté. Les évolutions récentes ont entrainé une certaine «chute » de statut des classes moyennes anciennes, celui-ci étant principalement fondé sur le capital symbolique lié au niveau d'éducation. Avec la dynamique récente de l'investissement public dans le secteur de l'éducation, la classe moyenne, qui avait en grande partie préservé son statut à travers celle-ci, s'est retrouvée déclassée et a subi un rapprochement avec la classe moyenne-inférieure. Simultanément à cela, en raison de la baisse de qualité des écoles publiques, les classes moyennes laïques s'orientent désormais vers les établissements privés et on observe également une prolifération des écoles à tendance islamique visant les nouveaux riches conservateurs. Le capital culturel, en particulier sous forme d'éducation et de maitrise de l'anglais, reste essentiel pour participer aux nouvelles formes de vie plus cosmopolites. Par-là, des formes de mimétisme/convergence se font jour entre les fractions de la classe moyenne.

Comme cela a déjà été mentionné plus haut, la piètre qualité de l'enseignement public primaire et secondaire au Brésil contraint les familles de la classe moyenne à s'orienter vers les établissements privés. L'amélioration de la qualité de l'enseignement public est donc cruciale, notamment pour les familles du bas de la classe moyenne qui n’ont pas nécessairement les ressources financières pour assurer une scolarisation dans le secteur privé à leurs enfants. La logique est très différente concernant l'enseignement supérieur. Dans les filières des universités publiques (surtout les universités fédérales et à un moindre degré les universités estaduales), les places étant peu nombreuses et les concours d'entrée rigoureux, ce sont les jeunes issus des établissements privés qui ont les plus fortes chances de suivre un enseignement supérieur public de qualité où les professeurs perçoivent des rémunérations de bon niveau selon les comparaisons internationales. Le strict numerus clausus en vigueur à l'entrée des universités publiques a pour conséquence de refouler une grande partie des étudiants vers les facultés privées constituant un marché lucratif et dont les évaluations officielles montrent qu'elles sont de moindre qualité. Là aussi, les familles de la classe moyenne expriment des attentes en matière d'élargissement de l'accès aux universités publiques.

Au Vietnam, le gouvernement a fait des efforts importants d'investissement dans l'éducation de base pour tous, avec des résultats impressionnants sur la scolarisation primaire et secondaire inférieure. Toutefois, la « socialisation de l'éducation » a conduit à ce qu'une part importante des dépenses scolaires (transports, manuels, uniformes, contributions volontaires) a été dévolue aux parents. La mauvaise qualité des écoles publiques et les coûts élevés de l'éducation que les 
ménages pauvres doivent supporter conduisent à de faibles taux de scolarisation aux niveaux non obligatoires (lycées et supérieur). Enfin, le management des compétences est défaillant, les enseignants gagnant de bas salaires par rapport au secteur privé manquant de motivation pour améliorer leurs compétences. Au lieu de cela, ils organisent des cours privés pour les enfants dont les familles peuvent se permettre ces dépenses supplémentaires. Les parents de tous les groupes de revenu considèrent que des cours supplémentaires sont essentielles à la réussite scolaire, les familles à revenu moyen et élevé allouant plus d'un tiers de leurs dépenses d'éducation au tutorat privé au niveau secondaire. La faible qualité de l'école publique combinée aux stratégies de contournement mises en place par les ménages les moins vulnérables pourrait accroître à terme l'inégalité entre les familles pauvres et les familles bien nanties (OECD, 2014). Or, le système éducatif tend à être fortement inclusif et largement équitable, notamment au stade de l'enseignement secondaire inférieur. Il ne devient exclusif, de qualité moyenne et inéquitable seulement au niveau supérieur et les rendements de l'éducation restent remarquablement faibles par rapport aux normes internationales (OECD, 2014). Cela signifie qu'aucun effort spécifique n'a jusqu'ici été fourni par le gouvernement pour doter les classes moyennes en croissance d'un système d'enseignement supérieur de qualité tout aussi inclusif. Cependant, de profondes réformes de l'enseignement supérieur et de la formation professionnelle visant à améliorer la qualité de l'enseignement et de l'apprentissage, ainsi que les installations scolaires, sont essentielles pour que le système éducatif vietnamien puisse répondre aux exigences croissantes d'une économie en mutation rapide

En Côte d'Ivoire, la période d'insécurité politique et de guerre civile a fait singulièrement reculer les progrès précédemment enregistrés en éducation. Le système éducatif fonctionne mal, les indicateurs sont aujourd'hui au rouge : au niveau quantitatif, l'espérance de vie scolaire n'est que de 7,7 ans, comparativement à une moyenne africaine de 9,7 ans, et de 12 ans pour les pays à revenus intermédiaires en 2015. Au niveau qualitatif, une étude réalisée en $3^{\text {ème }}$ année du primaire en 2012 montrait que $87 \%$ des élèves ont un niveau faible ou très faible en français et $73 \%$ en mathématiques, avec une forte détérioration entre 1996 et 2009 (Banque Mondiale, 2017 ; OCDE, 2016). La situation contemporaine se caractérise ainsi par une configuration singulière opposant un système éducatif fragilisé, y compris dans le secteur urbain, et une forte demande sociale pour l'éducation, en particulier chez les classes moyennes. Si la Côte d'Ivoire souhaite devenir un pays émergent, la Banque mondiale (2017) estime qu'elle devra doubler son budget lié à l'éducation d'ici 2025 et passer à la vitesse supérieure pour rattraper le retard accumulé. Dans ce contexte, les classes moyennes ne semblent pas constituer une cible particulière des politiques publiques. Dans un contexte de rareté des ressources, la priorité est clairement à l'accès à l'éducation pour les plus pauvres. Ceci rapproche la Côte d'Ivoire du Vietnam. Comme le souligne l'un de nos interlocuteurs au Ministère de l'Education Nationale dans le cadre d'un entretien institutionnel: "J'aurai tort de cibler ces catégories. Parce qu'elles se débrouillent suffisamment déjà. Ce que je veux dire c'est qu'elles tirent le meilleur de ce qui existe déjà. Les établissements privés constituent alors pour le gouvernement une opportunité pour pallier le déficit de l'offre éducative. L'offre privée s'oriente de facto vers ces ménages capables de subvenir aux frais de scolarité, souvent en sacrifiant une partie de leur bien-être ou de leurs projets d'investissement. 
Dans les quatre pays de notre étude, les classes moyennes expriment une aspiration à l'amélioration de la qualité des services de santé et d'éducation. La demande diffuse est celle d'une ambition plus grande de l'Etat pour accompagner et soutenir les désirs d'investissement dans le capital humain des groupes de revenu intermédiaire. Ceci peut passer par un accroissement de l'accès aux services de soin publics et gratuits pour les non-pauvres, ou bien par une amélioration de la qualité du service des écoles publiques. Face à ces demandes, la réponse de l'Etat est bien souvent de favoriser le développement d'une offre privée, structurée et officielle comme c'est le cas dans le secteur de l'éducation au Brésil et en Turquie, ou bien plus spontanée et informelle, comme c'est le cas au Vietnam et en Côte d'Ivoire où les enseignants augmentent leurs salaires en offrant des cours privés très recherchés par les familles de la classe moyenne.

\subsubsection{Des politiques limitées en matière de logement et de mobilité}

Le logement a été au cœur des revendications exprimées dans les mouvements sociaux de ces dernières années au Brésil. L’enquête qualitative a montré qu’un tiers des ménages interrogés souhaitaient déménager mais que des obstacles d'ordre financier rendaient difficile la réalisation de ce vœu. Dans le passif social accumulé au long des décennies par le pays, le logement occupe, par son déficit, une place centrale. Une étude récente a montré que les carences en matière de logement se concentrent dans les familles percevant les revenus les plus modestes : 83,9\% dans les familles disposant d'au plus trois salaires minimums (FJP, MC, BID, PNUD, 2016). En outre, cette étude évalue à $9 \%$ le déficit en logements urbains pour l'ensemble du Brésil. Il résulte de cette situation que les logements disponibles voient leurs loyers augmenter régulièrement. Les loyers jugés excessifs, c'est-à-dire ceux dépassant 30\% des revenus familiaux, représentaient 7,5\% des logements en 2004 et 12,7\% en 2014 (IBGE, 2015). Ainsi, la croissance économique des années 2000 n'a fait qu'exacerber la crise du logement. Dans le même temps où elle a permis l'expansion de l'emploi formel, elle a rendu l'accès à un domicile plus difficile et plus coûteux, et les composantes les plus récentes et les plus fragiles de la classe moyenne ont évidemment pâti de cette situation. Au Brésil, les choix en matière de lieu de résidence et de logement sont reliés aux problèmes d'insécurité publique (voir ci-dessous). Au-delà du logement, les récriminations de la classe moyenne brésilienne portent sur la qualité des transports collectifs ainsi que sur les problèmes de densité du trafic urbain.

En Turquie, les choix résidentiels ont toujours été l'un des principaux repères reflétant l'écart entre les différentes classes de la société. Alors que vivre dans un appartement signifie faire partie de la classe moyenne, menant une vie moderne et urbaine, vivre dans un " gecekondu » (habitation construite sans permis) indique l'appartenance à une catégorie inférieure, d'origine paysanne et rétrograde. Depuis 1990, on assiste à la multiplication des gated communities (cités fermées et sécurisées), avec une accélération dans les années 2000. Ces cités offrent une privatisation quasi-totale des institutions et des services - écoles, crèches, restaurants, hôpitaux, sécurité, etc. Les cités fermées situées à l'extérieur de la ville sont ainsi devenues la nouvelle tendance des classes moyennes en matière de logement. La mobilité urbaine ou inter-urbaine est également une aspiration forte des classes moyennes turques, aspiration qui va de pair avec celle de l'accès à un logement moderne et personnel. A l'échelle intra-urbaine, dans les agglomérations 
principales, la discrimination sociale est forte entre les utilisateurs d'automobiles et les autres, malgré un développement notable des transports publics en site propre. L'installation dans des complexes résidentiels aux périphéries des agglomérations n'est rendue possible que grâce à la possession d'au moins deux véhicules par ménage. En cela, à l'échelle urbaine, on est autorisé à parler d'un accès de plus en plus inégalitaire à la mobilité, les politiques de transports en commun peinant à compenser les inégalités liées à l'accès à l'automobile. Face à ces aspirations, la réponse politique a été volontariste. Depuis le début des années 2000, l'Etat poursuit son entreprise de promotion par l'accès au logement «moderne », entre autre par des transferts massifs de terrains publics, avec des moyens accrus par rapport aux décennies précédentes, et dans un souci d'ingénierie sociale de plus en plus prononcé. Entre 2003 et 2016, l'Etat turc a construit directement ou fait construire plus de 500000 logements et permis, grâce à des taux d'emprunt relativement bas, à des centaines de milliers de familles de s'installer dans des immeubles collectifs qui restent des symboles puissants de la modernité résidentielle et même culturelle. On a ainsi pu dire qu'avec ces programmes, les gouvernements AKP successifs avaient pour objectif de promouvoir "leurs" classes moyennes, "politiquement correctes". L'accès au crédit immobilier, qui demeure toutefois très limité, peut donc être perçu comme un des indices révélateurs d'appartenance à la classe moyenne.

En raison des restrictions sur les mobilités internes, le taux d'urbanisation reste inférieur à son potentiel au Vietnam. Pourtant, les autorités Vietnamiennes nourrissent, à travers les plans stratégiques à horizon 2025 et 2050, des ambitions importantes en termes de développement urbain. Des investissements dans les infrastructures de transports sont programmés pour compenser les faiblesses actuelles en termes d'infrastructures urbaines et interurbaines. L'amélioration des conditions d'habitat et des conditions de mobilité est aussi une revendication forte de la part du groupe de revenu intermédiaire vietnamien. L'investissement dans l'amélioration du logement familial est une priorité qui est apparue dans les entretiens qualitatifs. Comme dans tout régime socialiste, la politique de logement social occupe une place importante au Vietnam. La priorité des autorités a été de cibler les ménages ruraux et urbains pauvres. Concernant les classes moyennes urbaines, leurs attentes en matière d'amélioration de leur logement ou de localisation sont uniquement ciblées par une politique d'amélioration de la capacité de financement pour accéder au marché immobilier privé. En parallèle, les politiques publiques ont cherché à favoriser le développement d'un secteur privé de promotion immobilière pouvant répondre aux attentes des classes moyennes. Alors que les segments de revenu les plus élevés de la classe moyenne vietnamienne ont largement bénéficié de cette politique en termes d'amélioration des conditions de logement, la classe moyenne flottante, à la fois exclue du marché immobilier et du ciblage des politiques de logement social, est contrainte à un habitat informel. $\mathrm{Au}$ Vietnam, les transports sont importants pour travailler et pour la vie sociale et la possession d'un moyen de transport privé est un signe de réussite, même s'il se limite encore pour de nombreux ménages de la classe moyenne à la possession d'une ou plusieurs motocyclettes par famille. La croissance rapide des centres urbains a conduit à une demande forte de transports qui a été satisfaite avant tout par une expansion des modes de transport privés. Même si des plans de développement de modes de transport urbain existent dans les grandes villes, leur mise en place 
est trop lente. En conséquence, les transports collectifs ne sont pas forcément mis en avant par les ménages comme une solution à leurs problèmes de transports.

En Côte d'Ivoire, la question du logement est au cœur du programme d'Alassane Ouattara qui dans le cadre de son second mandat a fixé un objectif de 150000 logements d'ici 2020. Ces logements doivent être répartis entre des logements économiques et sociaux $(60 \%)$ et de haut et moyen standing (40\%). Comme le souligne un interlocuteur au Ministère de la Construction, du Logement, de l'Assainissement et de l'Urbanisme : «(...) aujourd'bui le Programme présidentiel de logements sociaux est exactement dédié à la classe moyenne ». Les attentes sont en effet importantes au regard des difficultés d'accès à des logements de qualité, en particulier à Abidjan. Pour autant, les critères définis pour bénéficier de ce programme laissent songeur quant à l'efficacité du ciblage "classe moyenne": les indépendants de l'informel urbain et rural en sont largement exclus faute de garanties bancaires. De plus, les souscripteurs doivent apporter un montant initial de $10 \%$ du prix total des logements, soit un montant minimum d'environ 1,5 millions de FCFA (l'équivalent, en moyenne, de dix années d'épargne d'un individu de la classe moyenne). A côté de cela, le transport est également un enjeu central pour des classes moyennes qui habitent des quartiers excentrés du centre d'Abidjan afin de bénéficier d'un loyer abordable. Les déplacements sont problématiques : l'autoroute unique reliant le centre d'Abidjan à Yopougon, commune typiquement classe moyenne de deux millions d'habitants est saturée pendant deux à quatre heures, matins et soirs. Pour l'heure, les nouvelles infrastructures (troisième pont à péage, nouvelles voies rapides de la Riviera Golfe, route de l'aéroport, Marina sur la lagune) sont perçues comme une vitrine de l'émergence, concentrées sur les quartiers riches. L'acquisition d'un véhicule personnel n'est généralement pas considérée comme une aspiration première, en particulier pour les strates basses, ce dernier étant hors de leur portée, les usagers faisant alors avec la diversité extrême et éparpillée d'offres de transports collectifs, essentiellement privés.

\subsubsection{Des aspirations fortement marquées par les contextes nationaux}

Certaines attentes spécifiques ont pu être identifiées pour certains pays. Ces attentes sont pour partie liées au contexte particulier du pays et pour partie liées aux caractéristiques des classes moyennes. Pour autant, la plupart d'entre-elles ne trouve pas de réponses politiques appropriées.

Au Brésil, la sécurité publique et la fiscalité sont des domaines pour lesquels la classe moyenne exprime des attentes fortes. Une partie de la déception de la population vis-à-vis des institutions et des hommes politiques provient des graves problèmes de sécurité publique dont souffre le pays et qui, à côté de la puissance croissante des réseaux et trafics de drogues, signe l'incapacité des pouvoirs publics quels que soient les gouvernements en place. En 2014, près de 60000 homicides ont été recensés, soit un taux de 29,1 homicides par 100000 habitants, plaçant le Brésil parmi les dix pays les plus dangereux du monde. Si ces données quantitatives témoignent des graves défaillances des pouvoirs publics au cours du temps, la violence et l'insécurité publique n'affectent pas indifféremment les diverses strates sociales. Les familles riches ou appartenant au segment supérieur de la classe moyenne y sont moins exposées car elles ont les moyens de se réfugier dans des ensembles immobiliers «bunkérisés » et gardiennés en permanence (condominios 
entourés de murs et barbelés, hauts immeubles disposant de tous les conforts - piscines, salles de sport, salles de jeux et de fêtes, garages, etc.) et circulent souvent dans des automobiles blindées. Les familles aux revenus plus modestes sont plus souvent victimes de cette insécurité permanente et généralisée.

La classe moyenne brésilienne exprime également de nombreuses critiques à l'encontre de la fiscalité. Le poids des prélèvements fiscaux des trois sphères de gouvernement (fédéral, estadual et municipal) atteint ces dernières années 37\% du PIB (OCDE, 2015). C'est un niveau comparable à celui des économies développées mais supérieur aux autres pays latino-américains. Dans son ensemble, cette fiscalité, du fait qu'elle ponctionne principalement les biens et les services, fait peser un poids jugé excessif sur les catégories sociales modestes et intermédiaires (IPEA, 2011). En 2013, sur le total de la collecte des impôts des trois sphères de gouvernement, 51,3\% portaient sur les biens et les services, 18,1\% sur les revenus et 3,9\% sur la propriété. C'est une matrice fiscale bien différente des pays du Nord, et même de pays sud-américains comme le Chili, le Pérou et la Colombie. De plus, alors que dans les pays de l'OCDE l'impôt sur le revenu correspond en moyenne à 8,5\% du produit fiscal, au Brésil il n'en représente que 2,7\%. Les taux marginaux sont peu élevés, la dernière tranche étant de 27,5\% au Brésil (contre 35\% en Turquie). L'iniquité ne se limite pas à une absence de progressivité des impôts, mais à leur franche dégressivité. Afonso et Castro (2012) mentionnent des travaux récents réalisés au sein de l'Université de São Paulo montrant que 49\% du revenu des familles appartenant au premier décile de revenus sont prélevés par l'autorité fiscale contre à peine $26 \%$ du revenu des familles relevant du dernier décile. Les ménages les plus riches sont également ceux qui bénéficient le plus des nombreuses déductions fiscales. Comme l'enquête qualitative a pu le montrer, ceci explique le sentiment très répandu au sein de la classe moyenne de devoir payer pour les autres catégories sociales : au profit des pauvres dans le cadre des transferts sociaux et en substitution des riches, qui sont formellement et notoirement sous-fiscalisés et qui parfois adoptent des comportements illégaux de sous-déclarations ou d'évasion fiscale pour réduire encore leur exposition à l'impôt.

L'enquête qualitative a révélé qu'au Vietnam, les membres du groupe intermédiaire de revenu ayant migré vers les grandes villes sont attachés à trouver une harmonie entre modernité et traditions, entre la vie urbaine et des retours fréquents dans leur village d'origine. Ils aspirent à des déplacements plus faciles et à une harmonie entre leur vie urbaine et la vie rurale de leurs parents ou ancêtres. La disponibilité pour la famille, et notamment pour les enfants, est un élément de qualité de vie revendiqué par certains chefs de ménage des classes moyennes. La sécurité n'est pas un problème pour les ménages des classes moyennes vietnamiennes, à l'exception des plus vieux. En revanche, beaucoup regrettent l'évolution des mentalités urbaines vers plus d'individualisme et moins de solidarité, plus de consumérisme et moins de spiritualité. La vocation culturelle de la classe moyenne, vue comme le vecteur de diffusion de nouveaux modes de consommation ou de comportement, est également soulignée dans les entretiens institutionnels. Les attentes autour de l'expansion de la classe moyenne sont donc importantes dans toute la société, mais les Vietnamiens attendent que la classe moyenne adapte les comportements qui la définissent (consommation, éducation, entreprise) à la culture et aux valeurs héritées de l'histoire du Vietnam. 
Dans le même sens, en Turquie, il faut souligner que la mobilité sociale ascendante vers la classe moyenne, lorsqu'elle entraîne un passage du rural à l'urbain, n’implique pas toujours une totale rupture avec le premier. Ceci est spécifiquement vérifié pour certaines agglomérations dont l'arrière-pays se caractérise par une activité agricole dense. Là, les classes moyennes urbaines n'ont pas rompu avec les villages et les petites villes dont elles proviennent. Certaines personnes, qui résident en ville, continuent en fait à se livrer à l'agriculture dans leur village d'origine. En dépit du discours récurrent sur la mort de l'agriculture, les nouveaux urbains n'ont pas abandonné l'agriculture et s'assurent même parfois l'essentiel de leurs revenus en poursuivant une activité agricole souvent destinée à la consommation de leur famille. La conservation d'un lien avec le village d'origine assure des ressources encore importantes pour faire face aux difficultés économiques inhérentes à la vie en ville. Et même les ménages qui ont un revenu plus élevé sont encore marqués, dans leur mode de vie, leurs valeurs et leurs systèmes de normes par l'univers rural dont ils sont issus et qu'ils tendent à perpétuer en ville. Plus avant, l'urbanisation et la mobilité sociale signifient d'une façon ou d'une autre l'adaptation à la vie dans les grandes villes et à la culture nationale dominante. La promotion sociale souvent liée à une migration vers les grandes villes du pays, à commencer par Istanbul, s'est bien souvent accompagnée de formes d'assimilation volontaire vécues par les parents de diverses origines ethniques. Leur mobilité sociale s'est ainsi accompagnée d'un processus de «turquification », très prégnant entre autre dans la fonction publique d'Etat, qui semble avoir joué un fort rôle intégrateur en entraînant une relativisation des allégeances ethniques et culturelles propres au milieu familial.

La Côte d'Ivoire présente la particularité, comparativement aux trois autres pays, de renouer avec un cycle de croissance forte après trente années d'instabilités socioéconomiques et une décennie 2000 marquée par un conflit militaro politique fort et la séparation en deux du pays. Cette situation se matérialise du point de vue des aspirations des classes moyennes par un soutien assez partagé à tout processus de retour à la paix qui permettrait d'arrêter le déclin économique et le délitement des structures de l'ordre public dans le pays. A cet égard, il faut noter qu'une grande avancée du gouvernement Ouattara a surement été de réorienter en quelques années l'image de la Côte d'Ivoire d'une rhétorique de l'humanitaire post-conflit vers une rhétorique de l'émergence. Pour autant, nos entretiens qualitatifs montrent que le traitement de la résolution du conflit et le sentiment d'une «justice des vainqueurs » laissent couver un sentiment de rancœur, bien que la peur d'une nouvelle crise politique tende à favoriser la position de statu quo. Dans ce contexte, l'apparition du phénomène " microbes », des enfants et adolescents (8-25 ans) qui agressent en bandes organisées, inquiète la plupart des ménages interrogés. Ce phénomène est né au moment de la crise post-électorale à Abobo, un quartier pauvre d'Abidjan, et tend à gagner les quartiers occupés par les classes moyennes (Yopougoun, Angré), où ils sévissent de plus en plus. Les populations des classes moyennes perçoivent à travers ce phénomène une forme de démission de l'Etat, et un sentiment d'insécurité renforcé par une faible confiance dans la police. 


\subsection{Des politiques publiques hésitantes : entre promotion différenciée des classes moyennes et instrumentalisation}

Les politiques publiques en faveur des «classes moyennes» apparaissent beaucoup moins significatives dans les quatre pays que ce que l'émergence de ce groupe laissait prévoir. En réalité ces politiques se différencient selon trois grands types (1), qui toutes visent à assurer la gestion politique d'une partie de la population potentiellement déterminante pour la gouvernance (2).

\subsubsection{Trois modèles de politiques publiques}

Si dans les quatre pays analysés, les classes moyennes formulent des attentes de politiques qui les cibleraient mieux, notamment en matière d'éducation et de protection sociale (cf. supra), il reste difficile d'identifier des politiques publiques qui leur soient explicitement dédiées. Ainsi, certaines politiques sectorielles potentiellement orientées vers ces catégories ne touchent en réalité que certains segments des classes moyennes alors que d'autres qui ne ciblent pas spécifiquement ces catégories ont des effets directs ou indirects sur elles.

\section{Des politiques publiques par défaut: " Pas de politique est une politique »}

L'une des évidences les plus claires de cette étude est que les autorités publiques gèrent en bonne partie les aspirations des groupes de revenu intermédiaires par l'absence d'intervention directe. Cette absence de politique est une politique en soi. Elle consiste à reporter sur le marché la gestion des demandes et aspirations de ces catégories qui expriment souvent leur souhait de ne pas voir l'état intervenir. En matière de santé, d'éducation ou de logement, les politiques incitatives ne ciblant que certains besoins et reportant sur les ménages «moyens» les choix décisifs se multiplient.

Si l'expression "instrumentalisation économique" est à prendre avec des précautions elle traduit surtout le constat commun aux quatre pays de politiques qui semblent plutôt chercher à favoriser, consciemment ou par défaut de moyens, la création d'une classe moyenne par le marché, par l'identité économique plus que par l'identité politique, et cela en particulier à travers la marchandisation de la consommation et des aspirations typiques de ces classes moyennes (éducation santé, logement).

La classe moyenne apparait alors comme une classe collatérale et à crédit, collatérale car elle est le produit des politiques anti-pauvreté (cf. infra), et à crédit car elle est la cible des politiques publiques de promotion et transformation de la consommation par le développement de nouveaux marchés (crédit, logement, éducation et santé) qui sont autant de domaines dans lesquels l'Etat ne s'engage pas pour favoriser le développement du secteur privé et l'attractivité des investissements étrangers. Avant d'être perçus comme des classes moyennes, ces groupes sociaux sont surtout vus comme des groupes de revenu dont la principale vertu est de soutenir l'économie par leur consommation immédiate et de soutenir ainsi le projet d'émergence, comme en Côte d'Ivoire ou au Vietnam. Ainsi le ministre du commerce ivoirien exprime clairement cette 
vision en déclarant le 24 mai lors d'une visite sur le chantier du nouvel hypermarché huppé Playce: "Te suis heureux de l'avancement des travaux qui se font à PlaYce Palmeraie Cocody (...) Tout ceci vient stimuler en fait la concurrence dans le secteur des distributeurs de produits alimentaires, des distributeurs de divers produits de consommation, qui sont destinés soit à la classe moyenne, soit à la majorité des ivoiriens »; ainsi, "la Côte d'Ivoire continue sa marche dans une dynamique d'aller de l'avant par rapport aux ambitions que le Chef de l'Etat s'est fixé pour faire de la Côte d'Ivoire un pays émergent" ${ }^{\prime 2}$. L'élargissement du périmètre de la classe moyenne à la « majorité des ivoiriens » traduit bien ici la volonté d'orienter les investisseurs vers des marchés nouveaux en cours de solvabilisation, constitués encore aujourd'hui de ménages aux revenus modestes mais qui commencent à aspirer à des formes de consommation primaire plus élaborée. Les “classes moyennes" sont donc encore en bonne partie, et de manière d'autant plus marquée que la société est plus pauvre et moins structurée socialement comme en Côte d'Ivoire, un mot performatif, permettant de créer des représentations de la réalité sociale qui ne sont que partiellement vraies mais qui orientent réellement les pratiques (investisseurs étrangers, bailleurs internationaux, etc.). Au Vietnam, le schéma de mobilisation de la classe moyenne est moins officialisé bien que présent dans les esprits, la plupart des acteurs institutionnels interviewés insistant sur le rôle, et sur l'exemplarité, des classes moyennes dans la poursuite du processus collectif de développement entamé depuis les réformes Doi Moi. Leurs comportements de consommation et leurs efforts doivent encourager les autres groupes sociaux à agir dans le sens du développement économique.

Les quatre cas étudiés nous semblent valider cette interprétation générale, bien qu’à des degrés différents. La classe moyenne n'est donc pas fabriquée politiquement, mais est le résultat, et en même temps la source, de l'expansion des marchés. Le développement des activités privées de services collectifs est soutenu par la demande de certaines composantes des classes moyennes des quatre pays en même temps qu'elle offre des opportunités professionnelles d'accès aux classes moyennes. Toutefois, la Turquie constitue une exception possible car une partie de la classe moyenne y est soutenue politiquement par un ensemble de politiques destinées à renforcer son patrimoine ou son niveau de capital humain afin qu'elle puisse rivaliser avec l'ancienne classe moyenne héritée de l'histoire.

\section{Des politiques qui s'adressent à des segments spécifiques des classes moyennes}

Certaines politiques sectorielles sont en réalité destinées à des segments des classes moyennes et non à l'ensemble de la catégorie comme telle.

Le Brésil fournit plusieurs exemples de ciblage différencié des classes moyennes. En matière de logement, le programme Minha Casa, Minha Vida (Ma Maison, Ma vie), financé par le gouvernement fédéral, vise à faciliter l'accès des familles à revenus modestes et moyens à la propriété immobilière par des crédits subventionnés. Si le programme vise avant tout les plus pauvres, les tranches inférieures de revenu de la classe moyenne en bénéficient également. En

${ }^{20}$ https://cnlvc.ci/2017/05/31/visite-ministre-commerce-souleymane-diarrassouba-a-playce-palmeraieouverture-prochaine/, page consultée le 13/11/2017. 
matière de protection sociale, ensuite, la création en 2013 d'une institution de prévoyance complémentaire des fonctionnaires et agents publics de l'Etat de Rio est une mesure pouvant directement concerner une partie de la classe moyenne puisqu'il était attendu qu'environ 30\% des près de 500000 fonctionnaires de cet Etat fédéré, soit la proportion de ceux jugés appartenir à la classe moyenne, cotisent à ce nouvel organisme de protection sociale cofinancé par l'Etat et par les cotisations ${ }^{21}$.

La Côte d'Ivoire témoigne également de politiques publiques présentées comme spécifiquement classe moyenne mais ne touchant réellement qu'un segment limité de celle-ci. Le programme de logements sociaux en est un bon exemple puisque ses critères d'éligibilité (garantie bancaire, montant de l'avance) conduisent de facto à un ciblage sur les strates hautes de la classe moyenne. De même jusqu'à présent les politiques sociales (assurance sociale) ont été ciblées sur les travailleurs formels du secteur public et privé bénéficiant d'un régime d'assurance obligatoire et systématique. Néanmoins, le projet de Couverture Maladie Universelle en cours vise à étendre la couverture sociale aux catégories vulnérables et pauvres. Pour l'heure ce projet tarde à se concrétiser. A l'inverse, les programmes de soutien à l'endettement pour accéder au marché immobilier privé au Vietnam ne ciblent que les classes moyennes supérieures.

Dans le même sens, plusieurs des pays étudiés ont adopté une réglementation du salaire minimum (plus ou moins contraignant selon son niveau par rapport au prix d'équilibre du marché du travail $)^{22}$. Si ce salaire minimum peut être considéré comme une mesure a priori destinée aux classes moyennes, cette politique ne couvre dans les faits qu'une partie de la classe moyenne, celle occupant un emploi formel. Pour certains pays comme la Côte d'Ivoire et le Vietnam, le poids de l'emploi informel dans la classe moyenne réduit largement la portée de cette mesure, même si un effet de signal (lighthouse effect) peut en partie jouer sur les revenus informels.

En Turquie, comme nous l'avons vu précédemment, une partie non négligeable des politiques de soutien ou d'accompagnement de la classe moyenne émergente (santé, logement, éducation) cible ouvertement la clientèle politique du pouvoir en place.

On voit ainsi que les politiques de soutien à la classe moyenne peuvent ne concerner que des fragments de celle-ci, très probablement en fonction des intérêts des dirigeants mais aussi des capacités de mobilisation des sous-groupes composant la classe moyenne. Cette diversité des politiques répond trait pour trait à la diversité des ménages constitutifs des classes moyennes.

21 Il s'agit d'un régime complémentaire dont l'adhésion est fondée sur le volontariat et dont les ressources proviennent à parts égales des cotisations des participants et de transferts de l'Etat. Ce dispositif vise à compenser la diminution du montant réel des pensions des fonctionnaires suite à des mesures d'alignement de leur régime de base au régime général. En dépit des campagnes d'information et d'adhésion, seuls $70 \%$ des individus ciblés ( $21 \%$ du nombre total de fonctionnaires) ont adhéré à ce régime complémentaire.

22 Il est très peu contraignant dans le cas du Vietnam par exemple. 
Quoique non explicitement destinées à la classe moyenne, certaines politiques "pro-pauvres" participent d'une consolidation par le bas de la classe moyenne, quand d'autres, adressées aux plus aisées, bénéficient également à celle-ci par effet de ruissellement.

En premier lieu, les politiques de sortie de pauvreté ont gonflé le bas de la distribution des niveaux de revenu intermédiaire et ont permis à un grand nombre de pauvres d'accéder à la classe flottante du bas de la classe moyenne. L'emblème de ces politiques pro-pauvres qui ont eu un impact sur la taille et la forme du groupe de revenus intermédiaires est la Bolsa Família au Brésil ${ }^{23}$. En accordant par individu une bourse mensuelle, elle a largement participé, avec l'augmentation simultanée du salaire réel, au mieux-être social de bien des familles brésiliennes pauvres et très pauvres. Toutefois, une fois les ménages sortis de la pauvreté, ils ne disposent pas nécessairement de programme social équivalent à la Bolsa Família leur permettant de maintenir ou d'intensifier leurs efforts d'investissement dans le capital humain. Des politiques similaires ont eu des effets positifs importants sur les dynamiques de sortie de pauvreté et d'accès aux classes moyennes au Vietnam.

De la même manière, certaines politiques d'appui aux indépendants et aux micro- et petites entreprises ne sont pas explicitement pensées comme relevant d'une stratégie de consolidation des classes moyennes mais souvent bien plus comme des politiques de lutte contre la pauvreté. Ceci est assez paradoxal puisqu'au regard de nos résultats ces politiques participent probablement à la consolidation de la classe moyenne compte tenu de la place qu'occupent ces activités productives dans les revenus de certains segments de la catégorie des revenus intermédiaires. Il en est de même des dispositifs d'investissement dans les infrastructures de l'information et de la télécommunication qui, bien que s'adressant théoriquement à tous, augmente en réalité les opportunités d'inclusion économique et sociale des classes moyennes de façon plus significative que les autres groupes, notamment les pauvres, du fait des niveaux plus élevés d'équipement et de compétence. C'est par exemple le cas au Vietnam, où de l'aveu de nombreux ménages interrogés dans le cadre de notre enquête, les NTIC ont permis de concilier mobilité et migrations professionnelles vers les villes et maintien des liens sociaux familiaux garantissant l'équilibre individuel et la prise en charge des enfants.

Au Brésil, compte tenu de la crise économique qui impacte le pays depuis 2014-2015, les différents niveaux de gouvernement ont procédé à des ajustements de réglementation visant les

23 Il s'agit d'un mécanisme de transferts directs de revenus sous condition de fréquentation scolaire et de vaccination des enfants des familles bénéficiaires. Fin 2013, il bénéficiait à 14 millions de familles, soit environ 50 millions d'individus pour un montant de 24 milliards de Reais correspondant approximativement à $2 \%$ des dépenses budgétaires fédérales (Lustosa et Fauré, 2013). Il vise à éradiquer l'extrême pauvreté (revenu mensuel familial par tête jusqu'à 70 Reais) et la pauvreté (revenu mensuel familial par tête de 70 à 140 Reais). 
classes moyennes impactées par le chômage qui tendent à opter pour le statut de microentrepreneur individuel (Micro Empreendedores Individuais). Un représentant d'une institution nationale d'appui aux micro et petites entreprises qui a été interrogé dans le cadre de l'enquête qualitative « institutions » confirme que les personnes qui appartenaient à la classe moyenne et qui se retrouvent au chômage (ou même celles qui ont encore un emploi devenu très incertain) expriment également de fortes et nombreuses demandes pour monter leur affaire sous ce statut, que ce soit dans le secteur du commerce alimentaire ou dans le domaine des prestations de services (consultance, comptabilité, assistance technique, alimentation rapide, etc.). En Côte d'Ivoire, les entretiens institutionnels ont également permis d'identifier des initiatives en cours qui visent spécifiquement ces indépendants de l'informel. Par exemple, l'amnistie fiscale décrétée par les autorités ivoiriennes au cours de l'année 2016 pour inciter les entreprises informelles à s'enregistrer. D'autres initiatives, relatives notamment au développement de produits de couverture sociale pour les travailleurs indépendants du secteur informel, tardent à se concrétiser. Deux régimes de prévoyance et de retraite à destination des travailleurs indépendants (avec un régime de base incluant des prestations de retraites et d'indemnités journalières et un régime de retraite complémentaire par capitalisation pour les indépendants dont les capacités contributives sont plus élevées) sont en effet à l'étude.

En second lieu, un certain nombre de politiques sectorielles répondant en priorité aux attentes des plus riches bénéficient aussi aux classes moyennes. C'est particulièrement le cas en Turquie, par exemple via le déploiement par le gouvernement turc de politiques de promotion du tourisme national et des loisirs. Le temps des vacances est un temps social qui fait et dit la différence sociale encore fortement en Turquie. Il y a ceux qui partent en vacances et les autres. L'Etat turc a une politique d'incitation aux vacances par la banalisation discursive du moment des vacances, l'organisation du calendrier des vacances, mais plus encore par une politique très favorable à la construction des résidences secondaires. Si le tourisme national constitue un marqueur clé de l'accession à la classe moyenne, la mobilité internationale demeure un indicateur distinctif de l'appartenance à la classe moyenne supérieure. Par ailleurs les loisirs, largement pratiqués dans les centres commerciaux, lieux de loisirs et de divertissement indissociables des pratiques de consommation des classes moyennes, constituent un autre secteur que l'Etat a contribué à organiser ces dernières décennies, dans un double souci (porteur de contradictions) de contrôle moral, d'une part, et de délégation au privé, d'autre part.

Les politiques de sécurité en Turquie témoignent également de cette logique (et ce n'est pas forcément une spécificité turque). Elles sont prises en charge par l'Etat Turc depuis les années 1990, parallèlement à une privatisation des services de sécurité, les deux dynamiques n'étant pas incompatibles. Au cours des années 2000, différentes lois ont été adoptées qui ont permis la structuration d'un secteur de la sécurité, sous le contrôle des institutions publiques chargées de cette mission (au premier chef desquelles le ministère de l'Intérieur). La plus importante de ces lois adoptées en juin 2004 (loi 4188) institue ce secteur qui va devenir ensuite à la fois pourvoyeur d'emplois formels permettant d'accéder à la classe moyenne, et de services collectifs privés aux classes moyennes et supérieures, en lieu et place de l'état. C'est en fait l'Etat au sens large qui est devenu le plus gros client de services privés de sécurité. 
Une dernière illustration de politique sectorielle ayant un impact sur les pratiques et attentes des classes moyennes, sans être spécifiquement dédiée aux classes moyennes, est la priorité accordée par l'Etat turc depuis les années 1950 à l'intensification des mobilités à l'intérieur du pays dans le transport terrestre, d'abord, puis, plus récemment, par le transport aérien. Ce n'est que plus récemment (après 2004) que le transport ferroviaire a fait l'objet d'une politique de relance, sans que pour autant le retard soit rattrapé et que la connotation sociale négative attachée à ce mode de transport soit encore effacée. La mise en place de lignes à grande vitesse et à prix élevés, qui s'opère progressivement, devrait changer la donne quant au profil social des utilisateurs du train. $\mathrm{Au}$ Brésil, c'est avant tout autour de la promotion du transport aérien que les politiques de transport impactent les comportements de classes moyennes.

Les classes moyennes de nos quatre pays ne sont pas la cible spécifique de politiques publiques qui leur seraient explicitement dédiées, à la différence des pauvres qui bénéficient depuis quelques années de politiques fortes destinées à améliorer leur sort. En revanche, certaines strates ou types de ménages peuvent devenir la cible de politiques particulières, cela permettant de pacifier des revendications des sous-catégories à forte capacité de mobilisation ou de booster des parties plus pauvres pour conforter la légitimité du régime. Enfin, les catégories intermédiaires de revenu peuvent bénéficier des effets de politiques sectorielles non ciblées (transport, sécurité, etc.).

\subsubsection{Des politiques ... politiques (policies make politics) : l'instrumentalisation politique des classes moyennes}

Certaines politiques ont pu être présentées comme des réactions aux demandes des classes moyennes. On pense notamment aux programmes anti-corruption au Brésil ou en Turquie, qui sont présentés comme des réponses aux revendications des classes moyennes les plus exposées aux injustices et aux coûts de la corruption, mais qui, bien souvent, répondent à des objectifs politiques de nettoyage des oppositions, comme on a pu également le voir récemment en Chine. Dans les pays de notre échantillon, la mobilisation par le gouvernement du concept de classe moyenne est motivée par des objectifs politiques de nature assez différente.

Si les autorités brésiliennes se sont résolument emparées du thème de la classe moyenne, par exemple, c'est qu'elles ont vu en lui un moyen simple et efficace, à usage interne, de montrer la légitimité et l'efficacité des orientations impulsées depuis une dizaine d'années. Au plan extérieur, ces actions permettaient de prouver l'appartenance pleine et entière du Brésil au club des pays émergents et de pouvoir ainsi revendiquer, avec eux, l'établissement d'un nouvel ordre dans les relations internationales. La sensibilité de ces enjeux explique que le gouvernement fédéral ait multiplié les discours, au Brésil et dans les arènes internationales, pour vanter cette réussite brésilienne comme a pu le faire l'ex-présidente Dilma Roussef au Forum économique mondial de Davos en janvier 2014.

De façon similaire, les retournements du régime d'Erdogan au sujet des classes moyennes sont une bonne illustration des tentations des gouvernants des pays en développement d'instrumentaliser à des fins politiques la classe moyenne ou certaines de ses composantes. Même 
si dans son discours la politique économique kémaliste repose sur la fiction d'une société sans classe, fusionnelle, organique et solidaire, dans les faits cette politique n'en a pas moins fait sienne l'objectif d'instituer une bourgeoisie nationale indépendante. Plus tard, dans la Turquie qui avait réalisé son entrée accélérée dans l'économie de marché au cours des années 1980, le luxe symbolisé par les biens importés et la consommation ostentatoire ont été analysés comme un effort pour acquérir un statut de «Turcs blancs », manière pour ces derniers de se distinguer des «foules noires ». Très récemment, la popularisation par les médias du concept de classe moyenne s'est faite à l'occasion des événements de Gezi (ou du soulèvement de Gazi) en 2013. Comme si la question soulevée par Gezi était celle de la constitution des classes moyennes en acteur politique à part entière. Aussi, à ce moment, la question des « potentialités politiques » des classes moyennes fut abondamment abordée. Comme dans d'autres pays, l'émergence d'une classe moyenne en Turquie s'accompagne d'un désengagement non négligeable de l'Etat qui facilite l'offre de marché à destination des classes moyennes et incite à la marchandisation de la demande des classes moyennes, en matière d'éducation par exemple (cf. supra). Par ailleurs, les politiques de santé et d'éducation de l'AKP, dirigées contre un segment privilégié des classes moyennes, ont été très bien reçues du fait de leurs visées apparemment égalisatrices. Ces politiques ont puissamment contribué aux succès électoraux répétés de l'AKP. Tout est en effet mis en œuvre par le pouvoir pour capter les voix des classes moyennes émergentes : offre de logements abordables et réservés, accès à la santé, éducation privée soutenue publiquement, etc.

Rien ne permet de mener ce genre d'analyse en Côte d'Ivoire. Dans la sphère politique ivoirienne, la notion de classe moyenne est peu présente. Elle n'apparaît qu'une seule fois dans le Plan National de Développement 2016-2020, à la différence du concept omniprésent d'émergence économique, certes intimement corrélée à celui de classes moyennes, mais sans que ce lien soit clairement défini dans les discours politiques officiels. Il est donc difficile de parler d'instrumentalisation politique des classes moyennes même si, certaines politiques sectorielles commencent à afficher un ciblage "classe moyenne" (cas de la politique du logement), et si la politique générale vise à sécuriser au mieux les opportunités dont se nourrit l'expansion de cet ensemble de population.

Le Vietnam pourrait apparaitre comme une exception car les classes moyennes n'y sont ni explicitement, ni implicitement instrumentalisées par le pouvoir. Elles sont au contraire contournées ou ignorées par le gouvernement communiste du fait de la différenciation sociale qu'elles véhiculent et qui est fondamentalement contradictoire avec l'idéologie d'une société harmonieuse et sans classe. Toutefois, cette indifférence n'est qu'apparente car les politiques économiques et sociales sont orientées, sans le dire explicitement, vers la densification de ce groupe de revenu, notamment à travers la croissance économique. Si la classe moyenne est instrumentalisée, c'est, comme en Chine, afin de justifier implicitement la légitimité des réformes engagées vers l'économie de marché, qui sont profondément contradictoires avec les valeurs politiques portées par le parti communiste vietnamien.

Une interprétation possible de ces différentes stratégies politiques des politiques publiques relève du risque potentiel que représente la formation d'une classe moyenne cohérente. La constitution 
d'un groupe classe moyenne trop homogène et trop autonome peut signifier une capacité de contestation et de mobilisation susceptible de défier le pouvoir en place, voire de s'y opposer et de le déstabiliser en raison de son poids démographique considérable. Ceci pourrait ainsi expliquer la frilosité des pouvoirs politiques de certains des pays étudiés (Vietnam, Turquie) à conférer une identité politique à ces groupes, ou à certaines de ses composantes, en les institutionnalisant comme des cibles de politique publique. L'absence de politique globale vis-àvis des classes moyennes dans nos quatre cas pourrait alors traduire une méfiance du pouvoir à l'encontre d'une institutionnalisation des classes moyennes ou de parties substantielles et notamment les plus fortes d'entre elles. Au Brésil et en Turquie, les gouvernements cherchent à se constituer des soutiens parmi la population, y compris parmi la classe moyenne, pour consolider leur assise de pouvoir. Pour autant, la constitution d'une classe moyenne n'est pas en soi une priorité car elle risque de provoquer des remises en cause si elle dispose d'une unité de représentation, comme le notait Huntington (1968; Villegas, 2012) ${ }^{24}$. Les gouvernements peuvent favoriser l'enrichissement des gens des classes moyennes car cela leur rapporte des soutiens individuels mais cumulables. En revanche, ils ne favorisent pas la création d'une identité de classe des «classes moyennes » car ce serait générer un pouvoir de mobilisation intermédiaire pouvant saper leur propre pouvoir.

Pour conclure, notre analyse comparative montre donc que dans les quatre pays, les aspirations des classes moyennes sont essentiellement instrumentalisées, c'est à dire transformées en opportunités politiques ou économiques, plutôt que traduites en politiques publiques ciblées. Autrement dit, si il existe peu de politiques publiques spécifiquement dédiées aux demandes des groupes de ménages à revenu intermédiaire, ces groupes ne sont pas pour autant négligés par les gouvernements des quatre pays qui les intègrent à leur stratégie politique, et parfois économique, afin (i) de limiter les tensions par rapport aux autres groupes sociaux, notamment les pauvres, (ii) de légitimer certaines des options idéologiques sous-tendant leur action publique et (iii) de rallier, ne serait-ce que par inertie, leur soutien. On oscille ainsi entre l'instrumentalisation clientéliste des classes moyennes en Turquie et l'instrumentalisation à des fins de légitimation des options de politique économique au Brésil, voire même du régime en Côte d'Ivoire et au Vietnam.

\section{Conclusion}

Cette étude avait pour objectif de proposer un examen approfondi des classes moyennes, de leurs caractéristiques, comportements, aspirations et attentes, ainsi que des implications de leur essor en matière de design des politiques publiques. L'étude portait sur quatre pays appartenant aux quatre grandes régions en développement (Asie, Afrique, Moyen-Orient et Amérique Latine) et présentant des niveaux et trajectoires de développement différentes ${ }^{25}$. Des traits communs comme des enjeux spécifiques à chacun des quatre pays étudiés ont pu être identifiés. Ces

24 Ces rapports politiques ambivalents peuvent expliquer par exemple les hésitations interprétatives de Huntington (1968 et 1991) sur l'impact politique des classes moyennes.

25 L'analyse approfondie des classes moyennes dans chacun des pays considérés a donné lieu à la publication d'une monographie spécifique. 
éléments de convergence et de divergence peuvent se résumer en six résultats qui viennent, de notre point de vue, enrichir la littérature sur les classes moyennes dans les pays en développement.

Premièrement, l'étude a révélé que la délimitation de classes moyennes de revenu est délicate, particulièrement dans une optique de comparaison. Compte tenu des différences de niveau de développement économique entre les quatre pays, il s'est révélé impossible de définir un intervalle monétaire unique applicable aux quatre contextes. Si la définition de la borne supérieure a pu être harmonisée en recourant au 95ème percentile de la distribution du revenu, il n’en a pas été de même pour la borne inférieure puisqu'être «non-pauvre » renvoie à des réalités clairement différenciées dans les quatre pays. Après un examen approfondi de la distribution des revenus dans les quatre pays, le choix a été fait de distinguer deux groupes de pays : la Turquie et le Brésil pour qui la limite inférieure a été fixée à $10 \$$ par jour et par personne en PPA et le Vietnam et la Côte d'Ivoire pour qui cette limite a été fixée à $4 \$$. Sur cette base, l'identification des classes moyennes de revenu a permis de mettre en lumière des réalités distinctes puisque le poids des ménages appartenant au groupe de revenu intermédiaire atteint son maximum sur l'échantillon en Turquie $75,4 \%$ des chefs de ménage couverts par l'enquête représentative nationale) et un minimum de 26,5\% en Côte d'Ivoire. Le Brésil (61,4\%) et le Vietnam (72,5\%) sont dans une zone intermédiaire. Si la comparaison de ces pourcentages est risquée du fait d'intervalles différents, le faible poids de la classe moyenne de revenu observée pour la Côte d'Ivoire suggère l'existence d'une exception ivoirienne, probablement généralisable au continent africain.

Deuxièmement, l'analyse comparative des quatre pays a permis d'identifier plusieurs éléments communs à ces catégories intermédiaires de revenu nationales. Tout d'abord, en dépit de différences considérables de revenu, les classes moyennes s'apparentent bien à des classes de consommation ou de consommateurs, ce qui se traduit par des dépenses de consommation élevées qui se déplacent et se diversifient vers l'éducation, le logement, la santé ou encore les loisirs. Cette dynamique quantitative et qualitative de consommation traduit un certain nombre de comportements et d'aspirations communes à toutes ces catégories. En effet, une partie de ces classes moyennes, plus ou moins importante selon les pays, a sécurisé une capacité de consommation lui assurant la satisfaction directe de ses besoins immédiats. En résulte une capacité renforcée à se projeter dans le futur, à penser un futur différent du présent qu'il convient de maîtriser par des actions de renforcement des capacités du ménage. Ainsi, l'insistance sur le mérite, la promotion de soi et les efforts dans la réalisation de ce projet se traduit partout par une part élevée des dépenses privées d'éducation, par une appétence nette pour la formation des jeunes et enfin par des stratégies généralisées de diversification des revenus (investissements dans le foncier, l'immobilier, etc.), mais également par une volonté d'amélioration de l'habitat. Tous ces traits confirment en parallèle une forte tendance individualiste, qui se matérialise dans les trajectoires personnelles des membres de la classe moyenne passant généralement par une coupure avec les familles élargies, l'insertion dans des voisinages marqués par une faiblesse des variables identitaires ou communautaires et une relation 
assez ambigüe à l'Etat, prenant la forme d'un soutien par inertie de certains groupes de la classe moyenne ou bien d'une contestation désorganisée.

Troisièmement, au-delà de ces éléments de proximité, l'étude comparative souligne la forte hétérogénéité interne de chaque classe moyenne nationale, notamment au regard des différences significatives qui existent entre elles en termes de statut dans l'emploi, de niveau d'éducation ou de revenu. Chaque ensemble national se caractérise par l'existence de quatre à sept groupes bien spécifiques du point de vue de l'articulation des caractéristiques socio-économiques, l'importance et les trajectoires de ces différents groupes ne pouvant s'expliquer que par l'historicité des trajectoires propres à chaque société. Ainsi, les groupes des agriculteurs-planteurs et de l'informel en Côte d'Ivoire, des retraités au Brésil, des entrepreneurs anatoliens en Turquie ou les gros agriculteurs spécialisés au Vietnam ne trouvent pas d'équivalents dans les autres pays. Où que ce soit, au Brésil, en Côte d'Ivoire, en Turquie ou au Vietnam, les "gens du milieu" sont des "gens des milieux" dans le sens où ils appartiennent à des "milieux", conçus comme des espaces sociaux de solidarité, différents et présentant des écarts considérables en termes de vulnérabilité économique et sociale ou de prestige et sécurité de leur rang social. Vont s'y côtoyer éventuellement ruraux et urbains (Côte d'Ivoire, Turquie, Vietnam), gens de culture moderne et occidentalisée et populations plus conservatrices (Turquie), gens du monde officiel et de l'informel (Côte d'Ivoire, Vietnam), quasi-pauvres, petite aisance économique et quasi-riches. L'ensemble est fortement fragmenté, ce qui, combiné à un fort individualisme, pourrait expliquer la diversité des comportements sociaux et politiques et les difficultés à organiser la mobilisation collective. Ces situations conduisent à des rapports très spécifiques selon les contextes nationaux entre catégories intermédiaires de revenu et État, rapports bien éloignés de la collusion généralement proclamée des classes moyennes et du pouvoir démocratique.

Quatrièmement, l'hétérogénéité des classes moyennes se matérialise également par des situations et trajectoires différentes au regard notamment de leur stabilité et de leur vulnérabilité. Même si elles présentent des spécificités nationales issues de l'histoire de la stratification sociale de chaque pays, deux profils communs aux quatre pays peuvent être identifiés. Partout, des classes moyennes en flux, c'est à dire des néo-classes moyennes directement sorties de la pauvreté et pouvant y retourner en cas de choc sont juxtaposées à des classes moyennes en stock, héritières et accédant à ce niveau social par transmission de capitaux (social, financier, culturel), dont le statut est sécurisé. Les groupes de classes moyennes en flux sont constitués de ménages installés dans le groupe revenu intermédiaire, mais pas encore stabilisés, le plus souvent de première génération, typiques de ces classes en transition que Bourdieu caractérisait par "ce qu'elles ne sont plus et ce qu'elles ne sont pas encore". Ces néo-classes moyennes se rapprochent à certains égards du groupe souvent qualifié de floating class ou de strugglers dans la littérature et caractérisant des ménages oscillant en permanence entre pauvreté et petite prospérité. Les groupes de classes moyennes "en stock" sont des ménages stabilisés disposant d'une protection contre les risques et les aléas et d'une plus grande aisance pour saisir les opportunités dans un milieu qui est déjà le leur. Il est cependant utile de souligner que dans les quatre pays, certains groupes suggèrent la possibilité de trajectoires d'ascension socio-économique par l'informel qui 
peuvent se transformer en classe moyenne en stock grâce à des mécanismes de reproduction intergénérationnelle contraints tout de même par un niveau assez faible de revenu et par une activité en marge des circuits officiels, de l'assurance sociale, ou de la bancarisation.

\section{Cinquièmement, les différentes classes moyennes nationales entretiennent des rapports ambigus et complexes avec l'Etat, le pouvoir et les politiques publiques.}

D'un côté, l'étude constate l'absence de politique officielle de promotion de la classe moyenne en tant que groupe social dont l'influence pourrait être reconnue stratégique dans la trajectoire présente et future de développement économique. En dépit de la forte présence rhétorique des classes moyennes dans les discours officiels des pays étudiés (à l'exception de la Côte d'Ivoire), peu de cas de politique publique ciblant effectivement les classes moyennes dans leur ensemble ont été identifiés. S’il n’y a pas de politique s'adressant globalement à la "classe moyenne", il y a bien des politiques sectorielles ciblant certains segments de la classe moyenne, et notamment les groupes les plus vulnérables. Il y a aussi des politiques sectorielles visant d'autres groupes qui, de manière indirecte, bénéficient à certaines composantes des classes moyennes. Certaines politiques "pro-pauvres" participent ainsi d'une promotion par le bas de la classe moyenne quand d'autres politiques, souvent adressées aux plus aisées, offrent des opportunités de consolidation du statut par le haut de la classe moyenne.

D’un autre côté, la très forte hétérogénéité socio-économique des classes moyennes nationales des quatre pays limite leur capacité d'organisation collective et d'influence politique. Les intérêts des sous-groupes qui composent la classe moyenne peuvent ainsi être en concurrence ou être mis en concurrence par des enjeux et des acteurs politiques. Une telle situation peut expliquer l'incapacité de tous ces groupes "du milieu” à s'unir pour défendre de manière structurelle et stabilisée dans le temps des intérêts communs. De grands mouvements peuvent exister, mais ils se lisent plus comme des mobilisations ponctuelles de groupes opportunément apposés que comme une fusion stabilisée d'intérêts.

Tout se passe comme si le pouvoir ne cherchait pas à générer une classe moyenne mais à accompagner l'émergence, par le marché, d'une diversité de groupes qui soient en position de le soutenir tout en n'ayant pas suffisamment de proximités pour risquer de s'unir contre lui. Dans les quatre pays, l'analyse des politiques publiques et des entretiens qualitatifs suggère que l'Etat y promeut la construction par le marché d'une classe moyenne de consommateurs, tout en se réservant la possibilité d'instrumentaliser ce groupe de revenu afin d'en dégager des avantages politiques ou économiques. Dans ces conditions, les classes moyennes semblent naitre essentiellement des opportunités offertes par un environnement économique porteur. L'Etat ne contribue qu'indirectement à leur émergence via l'ensemble de ses politiques, mais il ne fait le plus souvent (et très clairement dans les pays analysés) qu'accompagner ponctuellement une reconfiguration des groupes « du milieu » au profit de ses soutiens politiques du moment.

Sixièmement, les résultats obtenus dans le cadre de cette étude comparative permettent d'opérer un retour réflexif sur la notion de classe moyenne ainsi que sur les précautions que nous 
avions prises dans l'introduction pour aborder de manière non normative cette question dans le cadre des pays en développement. La notion de classe moyenne est tout aussi floue et discutable dans nos quatre pays qu'elle l'est dans la littérature qui a tenté de saisir cette réalité dans les pays industrialisés. Les gens du milieu sont d'abord des gens de différents milieux, que les niveaux et types de revenus, les modes de vie, les formes d'activité, les liens intergénérationnels, les représentations sociales et les affinités ne cessent de différencier et de scinder.

Deux grandes erreurs fondamentales dans les débats contemporains sur la définition et le rôle de la classe moyenne ont ainsi été identifiées dans cette étude. Premièrement, il semble que nombre d'études antérieures ont procédé à une transmutation du terme classe moyenne consistant à transformer, par un simple procédé de requalification linguistique, un groupe de revenu, construit de manière purement logique, quantitative et mécanique, en un groupe social très particulier pouvant aller jusqu'à s'identifier à une classe sociale. Il y a là une erreur méthodologique considérable. La notion de classes moyennes qui est mobilisée par les acteurs du développement économique est essentiellement fondée sur la transposition des expériences historiques des sociétés occidentales. Or, l'émergence des classes moyennes est un processus historique particulier à chaque société au point de déboucher sur des types différenciés de classes moyennes tant au Nord que désormais dans le monde en développement comme le confirment nos quatre études de cas. De la même manière que la notion de classe moyenne se décline différemment en Allemagne, en France, en Grande Bretagne et aux Etats-Unis, elle prend des configurations différentes dans nos quatre sociétés. Vouloir transposer une telle définition ou configuration sociale d'un contexte à un autre ailleurs expose donc les chercheurs à des erreurs d'interprétation considérables, comme le montrent les limites évidentes de la transposition de la notion de classe moyenne aux ménages africains dont l'enrichissement reste encore très relatif. Deuxièmement, comme nous l'avons montré dans ce travail, les classes moyennes sont non seulement très hétérogènes entre les différents pays en développement, mais elles le sont également au sein de chaque pays. Les sous-groupes qui en sont constitutifs ne forment pas un groupe cohérent rassemblé autour d'une conscience sociale ou politique spécifique. Ils ne s'identifient pas forcément à la notion implicite de la classe moyenne occidentale parce que leurs conditions de production sociale et économique est spécifique à chaque société. Postuler que les gens du milieu, parce qu'ils sont au milieu, sont des classes moyennes est déjà un pari hautement discutable et que nous remettons en cause pour ces quatre cas. Affirmer que parce qu'ils sont au milieu, ces groupes s'érigent automatiquement en défenseurs de la modernité universelle, de la démocratie et de la justice sociale est une prétention encore plus discutable. 


\section{Bibliographie}

Acemoglu, D., Robinson, J.-A. 2006, Economic Origins of Dictatorship and Democracy. Cambridge, Cambridge University Press.

ADB 2010, The rise of Asia's middle class, in ADB (ed.), Key indicators for Asia and the Pacific 2010, Metro Manila (Philippines), Asian Development Bank, 1-57.

AfDB 2011, The middle of the pyramid: Dynamics of the middle class in Africa, Report by the Chief Economist Complex, African Development Bank.

Adelman, I., Morris, C. 1967, Society, politics and economic development: a quantitative approach, The Economic Journal, 79(313): 160-163.

Afonso J.R.R., Castro K.P. 2012, Tributação: insuficiente reformar, necessário novo sistema, Controle, X(2): 11-31.

Alesina, A., Perotti, R. 1996, Income distribution, political instability and investment, European Economic Review, 40(6): 1203-1228.

Andrianampiarivo, T. 2016, Moderate prosperity, an adaptation of the middle class concept to a Malagasy rural area: The case of Itasy, Review of Social Economy, 75(1): 26-48.

Andrianjaka, R.R. 2017, Middle-class composition and growth in middle-income-countries, ADBI Working Paper, $\mathrm{n}^{\circ}$ 753, Asian Development Bank Institute.

Banerjee, A., Duflo, E. 2008, What is middle class about the middle classes around the world?, Journal of Economic Perspectives, 22(2): 3-28.

Banerjee A., Newman A.F. 1993, Occupational choice and the process of development, Journal of Political Economy, 101(2): 274-98.

Banque Mondiale (2017), «Le défi des compétences. Pourquoi la Côte d'Ivoire doit réformer son système éducatif ? », Rapport Situation Économique en Côte d'Ivoire, 4ème édition, Banque Mondiale.

Beresford, M. 1993, The political economy of dismantling the "bureaucratic centralism and subsidy system" in Vietnam, in K. Hewison, R. Robison, G. Rodan (eds.), Southeast Asia in the 1990s: Authoritarianism, Democracy and Capitalism, St Leonards: Allen and Unwin.

Birdsall N. 2010, The (indispensable) middle class in developing countries, in R. Kanbur, M. Spence (eds.), Equity and growth in a globalizing world, World Bank: Washington DC.

Birdsall, N., Graham, C., Pettinato, S. 2000, Stuck in tunnel: Is globalization muddling the middle?, Brookings Institution Working Paper, $\mathrm{n}^{\circ} 14$.

Birdsall, N., Lustig, N., Meyer, C.J. 2014, The strugglers: The new poor in Latin America?, World Development, 60(1): 132-146.

Bonnefond, C., Clément, M., Combarnous, F. 2015, In search of the elusive Chinese urban middle class: An exploratory analysis, Post-Communist Economies, 27(1): 41-59.

Bosc, S. 2008, Sociologie des classes moyennes, La Découverte, collection repères. 
Bourdieu, P. 1979, La distinction, critique sociale du jugement, Paris, Minuit.

Brunke, B., Van Dongen, B., \& Downey, W. 2013, Global topics 8 billion consumers. How to reach emerging market consumers with new strategies, Munich: Roland Berger Strategy Consultants.

Castellani, F., Parent, G. 2011, Being 'middle class' in Latin America, OECD Development Centre Working Paper, $n^{\circ} 305$.

Castellani, F., Parent, G., Zentero, J. 2014, The Latin American middle class. Fragile after all?, IDB Working Paper, $n^{\circ}$ 557, Inter-American Development Bank.

CFAO 2015, «Les classes moyennes en Afrique, quelle réalité ? quels enjeux ? », White Paper, CFAO-IPSOS-Bearing Point.

Chen J., Lu, C. 2011, Democratization and the Middle Class in China, the Middle Class's Attitude towards Democracy, Political Research Quarterly, 64(3): 705-719.

Chun, N. 2010, Middle class size in the past, present and future: A description of trends in Asia, ADB Working Paper, $\mathrm{n}^{\circ} 217$, Asian Development Bank.

Clément, M., Rougier, E. 2015, Classes moyennes et émergence en Asie de l'Est : Mesures et enjeux, Mondes en Développement, 43: 31-45.

CNC 2016, Pesquisa Nacional de Endividamento e Inadimplência do Consumidor (PEIC), Brasília.

Cogneau, D., Houngbedji, K., Mesplé-Somps, S. 2016, The Fall of the Elephant: two decades of poverty increase in Côte d'Ivoire (1988-2008), in Arndt, C., A. McKay, F. Tarp. (eds.) Growth and Poverty in Sub-Saharan Africa, New York, Oxford University Press

Contamin, B. et Fauré, Y.-A. 1990, La bataille des entreprises publiques en Côte d'Ivoire. L'bistoire d'un ajustement interne, Paris, Karthala.

Court, D., Narasimhan, L. 2010, Capturing the world's emerging middle class, McKinsey Quarterly, July.

Crédit Suisse Research Institute 2017, Emerging consumer survey 2017, Crédit Suisse.

ESCWA 2014, Arab middle class: Measurement and role in driving change, Economic and Social Commision for Western Asia, United Nations.

Darbon. D., Toulabor, C. (dir.) 2014, L'invention des classes moyennes africaines, Paris, Karthala.

De La Calle, L., Rubio, L. 2012, Mexico, a middle class society: Poor no more, developed not yet, Washington DC, Woodrow Wilson Center for Scholars.

De la Torre, A., Ize, A., Schmukler, S.L. 2012, Financial development in Latin America and the Carribean: The road ahead, Washington DC, Latin American and Caribbean Studies, World Bank.

Dobry, M. 1986, Sociologie des crises, Paris, FNSP.

Doepke, M., Zilibotti, F. 2008, Occupational choice and the spirit of capitalism, The Quarterly Journal of Economics, 123(2): 747-793. 
Easterly, W. 2001, The middle class consensus and economic development, Journal of Economic Growth, 6(4): 317-335.

Fauré, Y.-A. et Médard, J.-F. (dir.) 1982, Etat et bourgeoisie en Côte d’Ivoire, Paris, Karthala.

Ferreira, F.H.G., Messina, J., Rigolini, Lopez-Calva, L.F., Lugo, M.A., Vakis, R. 2013, Economic mobility and the rise of the Latin American middle class, Washington DC, World Bank.

FJP, MC, BID, PNUD, 2016, Déficit habitacional no Brasil. Banco de dados, www.cbicdados.com.br.

Freeman, R. 2007, The great doubling: The challenge of the new global labor market, in J. Edwards, M. Crain, A. Kalleberg (eds.), Ending poverty in America: How to restore the American dream, New York, The New Press.

Goldthorpe, J. 1980, Social mobility and class structure in modern Britain, Oxford, Clarendon.

Grimm, M., Knorringa, P., Lay, J. 2012 Informal Entrepreneurs in Western Africa: Constrained gazelles in the lower tier, World Development, 40(7):. 1352-1368.

Halbwachs, M. 1939, Les caractéristiques des classes moyennes, in R. Aron et al. (eds), Inventaires III. Classes moyennes, Paris, Alcan.

Hattori, T., Funatsu, T. 2003, The emergence of the Asian middle class and their characteristics, The Developing Economies, 41(2): 140-160.

Hoefel, F., Kiulhitzan, D., Broide, J., Mazzarolo, M. 2015, Mapping the mindset of Brazil's notso-new middle class consumers, McKinsey Quarterly, February.

Huntington, S. 1968, Political order in changing societies, Yale University Press (réédition 2006).

Huntington, S.P. 1991, The third wave: Democratization in the late twentieth century, Norman (OK), University of Oklahoma Press.

IBGE, 2015, Sintese de Indicadores Sociais 2015, Rio de Janeiro.

IPEA, 2011, Equidade Fiscal no Brasil : Impactos Distributivos da Tributaşão e do Gasto Social, Brasília, Comunicados do IPEA, $\mathrm{n}^{\circ} 92$.

Jaffrelot, C. 2008, Why should we vote? The Indian middle class and the functioning of the world's largest democracy, in C. Jaffrelot, P. Van Der Veer (eds.), Patterns of middle class consumption in India and China, New Dehli, Sage.

Kharas, H. 2010, The emerging middle class in developing countries, OECD Development Centre Working Paper, $n^{\circ} 285$.

Kharas, H. 2017, The unprecedented expansion of the global middle class. An update, Global Economy \& Development Working Paper, $n^{\circ} 100$, Brookings.

Kroeker, L. 2016, The Kenyan middle class and responses to social security, in A. BoanadaFuchs, Y. Gez, J.M. Waldmüller (eds), (Re)searching Nairobi's middle class, Geneva, Kompreno Research Report, K0001.

Landes, D. 1998, The Wealth and Poverty of Nations, New York, Norton. 
Lipset, S.M. 1959, Some social requisites of democracy: Economic development and political legitimacy, American Political Science Review, 53(1): 69-105.

Loayza, N., Rigolini, J., Llorente, G. 2012, Do middle classes bring institutional reforms?, Economics Letters, 116(3): 440-444.

Lustosa M.C. et Fauré Y.-A. 2013, Changements institutionnels et politiques publiques au Brésil Aux sources de la réduction des inégalités sociales, de la pawreté et des disparités régionales, Cahiers du GREThA, $\mathrm{n}^{\circ} 25$, Université de Bordeaux.

Marx, K. 1887, Capital. Progress Publishers, Moscow, USSR, Translated: Samuel Moore and Edward Aveling, edited by Fredrick Engels.

Matsuyama, K. 2002, The rise of mass consumption societies, Journal of Political Economy, 110(5): 1035-1070.

McKinsey Global Institute 2006, From 'made in China' to 'sold in China': The rise of the Chinese urban consumer, McKinsey \& Company.

McKinsey Global Institute 2012, Urban world: Cities and the rise of the consuming class, McKinsey \& Company.

McKinsey Global Institute 2017, Lions (still) on the move, McKinsey \& Company.

Melber, H. 2016, The rise of an African middle class: Myths, realities and critical engagements, London, Zed Books.

Merrill Lynch Global Research 2016, Theme watch: Rise of bottom billions.

Milanovic, B., Yitzhaki, S., 2002, Decomposing the world income distribution: Does the world have a middle class?, Review of Income and Wealth, 48(2): 155-178.

Mills, C.W. 1951, White collar: the American middle classes, New York, Oxford University Press.

Moore, B. 1966, Social Origins of Dictatorship and Democracy: Lord and Peasant in the Making of the Modern World. Boston, MA: Beacon Press.

Murphy, K.M., Shleifer, A., Vishny R. 1989, Income distribution, market size and industrialization, The Quarterly Journal of Economics, 104(3): 537-564.

Ncube M., Lufumpa, C. (eds.) 2015, The emerging middle class in Africa, London, Routledge.

OCDE 2016, Examen multidimensionnel de la Côte d'Ivoire. Volume 1 : Évaluation initiale, Série Les voies de développement, Paris, OCDE.

OCDE, 2015, Etudes économiques de l'OCDE : Brésil, Paris, OCDE.

OECD 2014, Social cohesion policy review of Viet Nam, Paris, OECD.

Peichl, A., Schaefer, T., Scheicher, C. 2010, Measuring richness and poverty: A micro data application to Europe and Germany, Review of Income and Wealth, 56(3): 597-619. 
Pham Xuan Nam 2002, Some preliminary ideas on the change of social structure and classes in Vietnam during the transition to a socialist-oriented market economy, Vietnam Social Sciences, 6: $31-42$.

Porchmann M. 2012, Nova Classe Média? O Trabalho na Base da Pirâmide Social Brasileira, São Paulo, Editora Boitempo.

Prahalad, C.K. 2009, The fortune at the bottom of the pyramid: Eradicating poverty through profits, New Dehli, FT Press.

Pressman, S. 2007, The decline of the middle class: An international perspective, Journal of Economic Issues, 41(1): 181-200.

Ravallion, M. 2010, The developing world's bulging (but vulnerable) middle class, World Development, 38(4): 445-454.

Resnik, D. 2015, The political economy of Africa's emergent middle class: Retrospect and prospects, Journal of international development, 27(5): 573-587.

SAE, 2012, Commissão para Definição da Classe Média no Brasil (Relatório), Brasília.

SAE 2013, Perguntas e Respostas sobre a Definição da Classe Média, Brasília.

Schotte, S. 2017, The anxious and the climbers: Ambivalent attitudes towards democracy among South Africa's middle class, GIGA Working Paper, n³04.

Sick, K.P. 1993, Le concept de classe moyenne : notion sociologique ou slogan politique?, Vingtième siècle, 37 : 13-41.

Skocpol T. 1984, Visions and methods in historical sociology, Cambridge and New York, Cambridge University Press.

Skocpol T., Somers, M. 1980, The uses of comparative history in macrosocial inquiry, Comparative Studies in Society and History, 22(2): 174-194.

Song, J., Cavusgil, E., Li, J., Luo, R. 2015, Social stratification and mobility among Chinese middle class households: An empirical investigation, International Business Review, 25(3): 646-656.

Sosa Escudero, W., Petralia, S. 2010, I can hear the grass grow: The anatomy of distributive changes in Argentina, CEDLAS Working Paper, $\mathrm{n}^{\circ}$ 0106, La Plata (Argentina).

Stampini, M., Robles, M., Saenz, M., Ibarraran, P., Medellin, N. 2015, Poverty, vulnerability and the middle class in Latin America, IDB Working Paper, n 591, Inter-American Development Bank.

The Economist 2017, Land to the tiller, 425(9062), 47.

Thiriot. C. 2004, Penser la politique comparée: un état des savoirs théoriques et méthodologiques, Paris, Karthala.

Thompson, M.R. 2004, Pacific Asia after 'Asian values': Authoritarianism, democracy and good governance, Third World Quarterly, 25(6): 1079-1095. 
Thurlow, J., Resnick, D. and Ubogu, D. 2015, Matching concept with measurement: Who belongs to Africa's middle class? Journal of International Development, 27, pp.588-608.

Veblen, T. 1899, The Theory of the Leisure Class: An Economic Study of Institutions, New York: B. W. Huebsch.

Vidal, C. 1997, Du rêve au réalisme : des citadins sans illusions, 1970-1994, in B. Contamin, H. Memel-Fotê (dir.), Le modèle ivoirien en questions, Karthala et ORSTOM, Paris.

Villegas C.M. 2012, Revolution from the middle: Class power, democracy and middle class narratives in the Philippines, Venezuela and Ecuador, Ph.D in sociology, Brown University, May.

Weber, M. 1947, Theory of Social and Economic Organization, New York, Free Press, originally published in German in 1920.

Wilson, D., Dragusanu, R. 2008, The expanding middle: The exploding world middle class and falling global inequality, Goldman Sachs, Global Economics Paper, $\mathrm{n}^{\circ} 170$.

Wiemann, J. 2015, Sharing global responsibility: The role of the middle classes on the way to a just and sustainable global economy, European Journal of Development Research, 27(2): 205-2016.

Wietzke, B., Sumner, A. 2014, The political and social implications of the "new middle classes" in Developing Countries: A literature review and avenues for future research, International Development Institute Working Paper, $\mathrm{n}^{\circ}$ 2014-03. 
Les Papiers de Recherche de l'AFD sont disponibles sur: https://www.afd.fr/fr/ressources

\# 2018-78 CRAMER, C., J. DI JOHN and J. SENDER (2018), "Poinsettia Assembly and Selling Emotion: High Value Agricultural Exports in Ethiopia", AFD Research Pa pers Series, No. 2018-78, August.

\# 2018-79 BOBBA, M., L. FLABBI and S. LEVY (2018), “Labor Market Search, Informality and Schooling Investments", AFD Research Papers Series, No. 2018-79, September.

\# 2018-80 BOBBA KOUADIO, A.O., F.-J . AZOH, M.-L. BONNEL, S. MAÏGA et J . N'DRI (2018), “Les collèges de proximité en Côte d'ivoire au prisme de I'approche par les capabilités", Papiers de Recherche AFD, n²018-80, Septembre.

\# 2018-81 RANZATO, M., MORETTO, L (2018), "Servicios de suministro de agua y energía y de tratamiento de residuos: Desarrollo de los comunes urbanos en el Sur Global mediante la coproducción de servicios", Papiers de Recherche AFD, $n^{\circ}$ 2018-81, Octubre.

\# 2018-82 CAVE, J. (2018), “En busca de los comunes urbanos: La gestión conflictiva de los residuos en Brasil", Papiers de Recherche AFD, $n^{\circ} 2018-82$, Octubre.

\# 2018-83 LEYRONAS, S., BAMBRIDGE, T. (2018), “Comunes y desarrollo: un enfoque renovado frente a los retos mundiales", Papiers de Recherche AFD, $n^{\circ}$ 2018-83, Octubre.

\# 2018-84 HAZAN, M., R. NOVELLA et C. ZANUSO (2018), "Aspirations, attentes et réa lités de la jeunesse dans un Etat fragile : le cas haïtien", Papiers de recherc he AFD, $n^{\circ}$ 2018-84, Novembre.

\# 2018-85 KUEPIE M. (2018), “Does family size hinder family living standards? Evidence from a quasi-experiment in Madagascar", AFD Research Papers Series, No. 2018-85, December.

\# 2019-86 CABRERA, J. E. (2018) "Les résea ux et la fragmentation urba ine : le cas des petis opérateurs des services d'eau à Cochabamba", Papiers de Recherche AFD, n²019-86, Décembre.

\# 2019-87 LEYRONAS, S. and N. LEG ROUX (2019), “Commons: Towards a New Narrative on Development Policies and Practices?", Papiers de Recherche AFD, No. 2019-87, January.

\# 2019-88 NILSSON, B. (2019), “Education and migration: insights for polic yma kers", AFD Research Papers Series, No. 2019-88, J a nuary. 